\title{
Description and Analysis of the Second Spectrum of Chromium, Cr II
}

\author{
Carl C. Kiess
}

\begin{abstract}
The analysis of $\mathrm{Cr}$ II presented in this paper is based on new wavelength measurements and estimated intensities, and on new observations of the Zeeman effect. Of approximately 2,100 lines listed in the tables, 89 percent have been classified as combinations of the levels of 48 even terms with levels of 51 odd terms. The even terms arise from the electron configurations $3 d^{5}, 3 d^{4} 4 s$, and $3 d^{3} 4 s^{2}$, the odd terms from the configuration $3 d^{4} 4 p$. No odd terms from the configuration $3 d^{3} 4 s \quad 4 p$ have been established. High even terms from the excited configurations $3 d^{4} 4 d$ and $3 d^{4} 5 s$ have been found that form Rydberg series with terms from the low even configurations. From them an ionization potential of 16.49 electron volts has been derived for the work required to convert a singly ionized $\mathrm{Cr}$ atom in the $a{ }^{6} \mathrm{~S}_{21 / 4}$ state to a doubly ionized atom in the $a^{5} \mathrm{D}_{0}$ state. The terms of the $3 d^{3} 4 s^{2}$ configuration lie high in the energy scale, and only a few relatively faint combinations between them and the odd terms have been found.
\end{abstract}

\section{Introduction}

The analysis of the second spectrum of chromium, presented in this paper, represents an attempt to classify its lines as completely as possible on the basis of present concepts of atomic structure. The work was started about 30 years ago and has been brought to its present status, from time to time, as new descriptive material for the spectrum became available. There are still some lines in the spectrum that resist classification; but at present it does not appear worth while to expend more time in order to identify them from existing data. The chief results of the investigation have been made known to those desiring them, as the work progressed, either through published notes or private communication, so that the main features of the analysis may be found in several well-known publications. But the new wavelength and Zeeman effect descriptions that have been made of the spectrum to improve and extend the analysis of the termstructure have not been published heretofore.

The inspiration for this work was found in the almost simultaneous discovery by Catalán ${ }^{1}$ [1], by Frl. H. Gieseler [2], and by C. C. and H. K. Kiess [3], of complex groups of lines, called multiplets by Catalán, in the spectrum of neutral chromium. At that time the available data for an analysis of the chromium spark spectrum were those referred to by Kayser [4] in volumes 5 and 7 of the Handbuch der Spectroscopie: wavelengths by Exner and Haschek, by Eder and Valenta, and by Cooper; Zeeman patterns by Miller, by Purvis, by Babcock, and others; and some temperature classifications by King. These data were sufficient for a beginning of the analysis; but further progress was greatly aided by the new and unpublished Zeeman patterns that were made available to me by H. D. Babcock, of the Mt. Wilson Observatory.

The status of the analysis in 1930 was described in a short paper by the writer [5], listing the known terms of $\mathrm{Cr}$ I and Crir. About the same time Krömer [6], and also Catalán [7], who had attacked

${ }_{1}^{1}$ Figures in brackets indicate the literature references at the end of this paper. the problem, made known their results, which were in substantial agreement with those worked out at the National Bureau of Standards. However, the grow th of atomic theory during the decade from 1920 to 1930 gave insights into the problems of atomic spectra that indicated their scope and suggested lines of approach in unravelling them. One of the first-fruits of the application of theory to Cr II was the prediction and finding by Kiess and Laporte [8] of the $a^{6} \mathrm{~S}$ term as the ground state of the singly ionized chromium atom. In the light of the theoretical expectations it became apparent that further progress in the interpretation of $\mathrm{Cr}$ II demanded a more accurate and extensive description of it than was then in existence.

\section{Experimental Frocedure}

The wavelengths and intensities listed in table 1 were derived from spectrograms made at the Bureau, at the Mt. Wilson Observatory, and at the Palmer Physical Laboratory of Princeton University. The first NBS observations were made with the Rowland concave grating ruled with 20,000 lines per inch. Owing to its low reflectance in the ultraviolet, this grating covered only the region above $2600 \mathrm{~A}$, with a scale of $1.8 \mathrm{~A} / \mathrm{mm}$ in the second order. Later, when a new concave grating with 30,000 lines per inch was received from R. W. Wood [9], the spectrum was resurveved from $2000 \mathrm{~A}$ in the ultraviolet to $8500 \mathrm{~A}$ in the near infrared, with reciprocal dispersions of 1 and $2 \mathrm{~A} / \mathrm{mm}$, respectively, in the second and first orders. Supplementary spectrograms of the ultraviolet were obtained from 1950 to $2400 \mathrm{~A}$ with the Hilger E1 quartz-prism spectrograph, and from 2200 to $3100 \mathrm{~A}$ with another Hilger instrument, which carries a large $60^{\circ}$ Cornu prism in combination with a $30^{\circ}$ reflecting prism in the E185 mounting.

The light sources were ares and condensed sparks between metallic chromium electrodes in air or in enclosures through which air or other gases could be kept flowing at reduced pressures. The enclosed arc was a reproduction of that described and illustrated by $H$. D. Curtis [10]. The ares were operated at 4 to 7 amp drawn from a $220-\mathrm{v}$ direct-current circuit. 


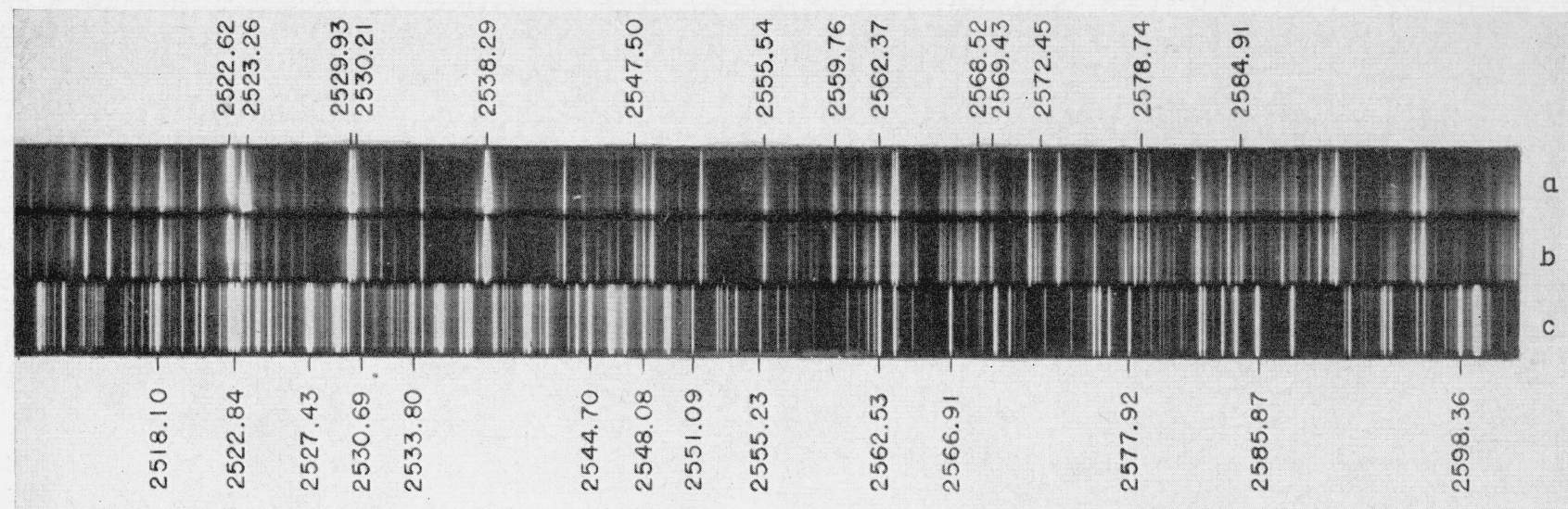

Figure 1. Cr II lines at atmospheric and reduced pressures: (a) 1 atm; (b) about 1/3 atm; (c) Fe arc spectrum.

The sparks were supplied by discharges from a battery of condensers rated at $0.006 \mu \mathrm{f}$ and charged from the $30,000-\mathbf{v}$ secondary coil of a transformer whose primary carried approximately 7 amp from a 110-v alternating-current circuit. Each exposure to the spark-in-air, with the Wood grating, was followed by an exposure to a condensed spark-discharge in the enclosure in which the pressure of the flowing gas was about $1 / 3 \mathrm{~atm}$. The purpose of this procedure is to sharpen certain groups of lines that appear diffuse and asymetrically broadened toward longer waves in the spark at normal atmospheric pressure. Its effectiveness is illustrated in figure 1.

The spark chamber is illustrated in figure 2. The enclosure consists of two Pyrex 6-in. bowl-type lead-in insulators, which can be clamped against gaskets attached to the cast brass ring, $A$. In the removable cell, $B$, in the front of the ring, either glass or quartz windows may be inserted. On one side of the ring at $C$ and $D$ are the inlet and outlet valves to the high and low pressure lines, and at $E$, on the other side, is the pinion for separating the electrode holders. On the back of the ring at $F$ is the sleeve by which it is attached to the vertical support along which it is raised and lowered. The connections to the electric circuit are through the binding posts at the small ends of the Pyrex bowls.

The spectrograms lent by A. S. King were obtained with the 15,000 lines-per-inch concave grating in the vertical Rowland mounting of the Mt. Wilson Observatory. They contain spectra of the arc and condensed spark in air covering the region from 3680 to $6600 \mathrm{~A}$ with a scale of 1.85 $\mathrm{A} / \mathrm{mm}$. The Princeton plates were made especially for this investigation by A. G. Shenstone with his vacuum spectrograph. This instrument carries a 2-m glass grating ruled by Wood with 30,000 lines per inch, and gives a dispersion of $4.2 \mathrm{~A} / \mathrm{mm}$. Light from arcs and sparks illuminated the full length of the slit so that some judgment of the ionization of the emitter of the lines might be formed from the distribution of intensity along the lines. The spectra extend from 1200 to $2200 \mathrm{~A}$.

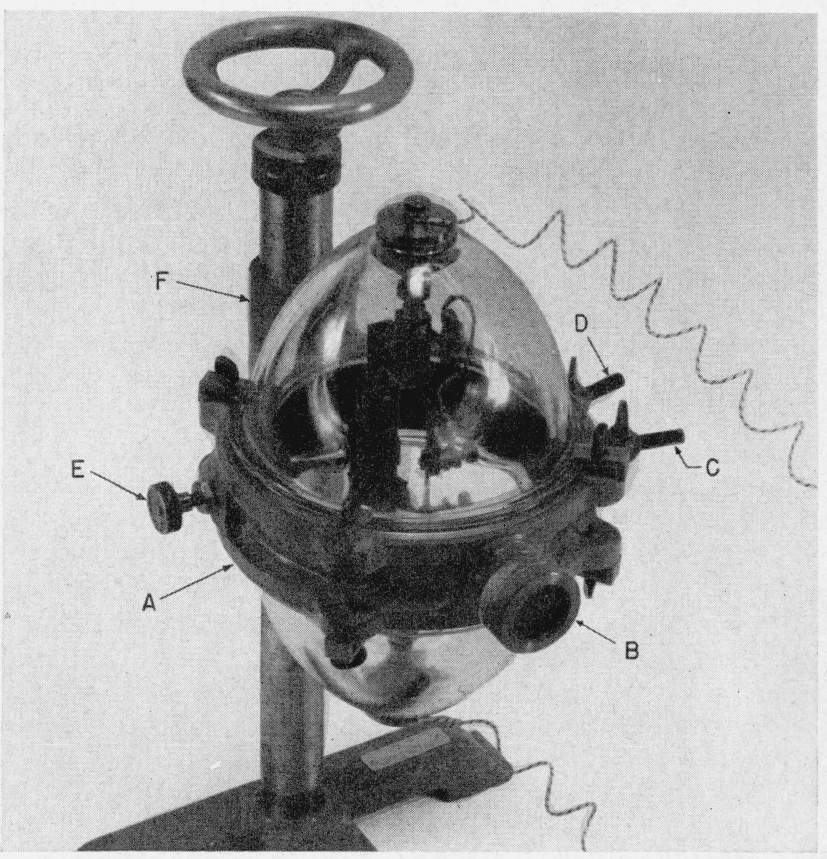

Figure 2. Enclosed lamp for operating sparks between metal electrodes at atmospheric and reduced pressures.

Babcock's published and unpublished Zeeman patterns were of inestimable value in the early stages of unravelling the chromium spectra. Yet as the analysis progressed, urgent need was felt for additional data of this kind, especially for fainter lines and those of shorter wavelength. When, therefore, the National Bureau of Standards acquired a large Weiss water-cooled magnet from the Société Genevoise, chromium was one of the first elements to be investigated. Fields of about 35,000 oersteds were attained in a gap of $5 \mathrm{~mm}$ between the ferro-cobalt poles when currents of 160 amp were flowing through the coils. The observations were recorded with the grating and prism spectrographs mentioned above and covered the wavelength range from 2300 to $6400 \mathrm{~A}$. When the Zeeman program was begun at Massachusetts 
Institute of Technology, a set of exposures to chromium was made, under the direction of G. R. Harrison, especially for this investigation; and later a supplementary set was made by W. F. Meggers.

\section{Results}

\subsection{Wavelengths and Intensitie;}

The wavelengths presented in the first column of table 1 were derived from measurements of the spectrograms described above. The tabulated values are means of two to eight determinations for all except a few of the lines. No line measured on only one spectrogram has been retained in the list unless its reality is verified from term-combinations. All the wavelengths in table 1 are air values. Although the wavelengths have been rounded off to the nearest $0.01 \mathrm{~A}$, yet it is believed that their accuracy exceeds this limit, except for the wide and hazy lines. Wherever possible, for such lines, the wavelengths were determined from spectra of the low-pressure source. The wavelengths of diffuse lines observed only in the spark-in-air were corrected for the redward shift due to pressure. Wavelengths measured below 2000 A are vacuum values. The lines of $\mathrm{Cr}$ II in this region that have been classified are listed in table 2 .

In the second column of the table are numbers and letters that indicate the strength and character of the lines. The letters have the following significance: $d=$ double, $g=$ ghost, $h=$ hazy, diffuse, $l=$ shaded toward longer waves, $s=$ shaded toward shorter waves, $w=$ wide, $W=$ very wide. In the last column the symbol $\dagger$ following a Zeeman pattern means that it was measured only on NBS spectrograms, all the others having been measured on MIT plates. The letters indicate the type of shading in unresolved patterns, thus: $A=1 \mathrm{~T}$, $B=\wedge 1, C=\wedge, D=\sqcap$. The letter $w$, with subscripts, indicates different degrees of widening of unresolved $p$ and $n$ components that do not have distinctive shading.

The intensities assigned to the lines are visual estimates that seek to express their strengths relative to the weakest ones measurable, which are given intensity 1. Such estimates are only qualitatively comparable between one region of the spectrum and another, owing to differences in sensitivity of the various kinds of photographic plates used to record the spectra. Thus, lines in the yellow region should not be compared with those in the ultraviolet bearing the same intensity numbers. However, certain quantitative relationships have been found to exist between the intensity scales used in this work and A. S. King's [11] and the intensities measured photometrically by Allen and Hesthal [12]. For about 65 lines in eight $\mathrm{Cr}$ II multiplets the latter authors give measured intensities that are in very good agreement with the theoretical relative intensities calculated for $L S$ coupling. For about 50 percent of these lines comparisons with King's estimated intensities show that the square roots of the measured intensities are quite accurately 1.1 times the estimated values. This is in strict harmony with Russell's [13] finding "that King's estimated intensities are very nearly proportional to the square roots of the actual intensities."

King's estimates refer to the strengths with which the lines appear in arc excitation, whereas the estimates in table 1 refer to their strengths in condensed sparks. For the region common to the two investigations the intensity scale of table 1 has a wider range than King's. For 32 lines to which King assigns intensities 1 to 5 the corresponding numbers in the table are 17.5 times greater; for 26 of King's lines in the intensity group $6-10$, the NBS values are 8.5 times greater; and for all of King's intensities above 10 the NBS values are 5.5 times greater.

\subsection{Zeeman Effects}

The magnetic patterns that have been measured on the NBS and MIT spectrograms are given in the last column of table 1 . These results portray the magnetic behavior of the radiating atoms at two field strengths, namely, 35,000 and 76,000 oersteds. Only those patterns from the NBS plates are reported that do not appear or cannot be measured on the MIT spectrograms. The two sets of observations are in very good agreement for all lines for which they yield resolved patterns. And the same may be said with respect to Babcock's measurements and those published by Krömer.

From these patterns were derived the $g$-values given in tables 3 and 4 for the $\mathrm{Cr}$ II levels. The $g$ 's are very nearly those required by Landé's theory for $L S$-coupling, especially for the levels of the low terms. In the quartet terms from the $3 d^{5}$ electronconfiguration, however, the levels are separated by very small intervals, much less than their splittings in the magnetic fields used in this work, and they are partially inverted so that the algebraic sum of the intervals differs little from zero. The Zeeman patterns observed for lines involving these levels are badly distorted owing to Paschen-Back interactions. The patterns exhibit striking dissymmetries in the intensities of the components, and their complexity is increased by the presence of transitions forbidden by the selection rule for inner quantum numbers. Nevertheless, it has been possible to resolve these patterns by the procedure followed by Kiess and Shortley [14] for O I and $\mathrm{N}_{\mathrm{I}}$ and to derive the $g$ values listed in table 3 for the perturbed levels. The lines of table 1 so affected are designated by the symbol P-B. The details of their interpretation will be presented in a subsequent paper.

\subsection{Term Structure of $\mathrm{Cr}$ II}

The spectrum to be expected theoretically for the $\mathrm{Cr}^{+}$ion is similar to those of $\mathrm{V}_{\mathrm{I}}, \mathrm{Mn}$ III, Fe IV, etc., which are characteristic of atoms and ions with 23 external electrons. All except five of these electrons are in closed groups. In the low and metastable states of the ion the five valence electrons 
form the even configurations $3 d^{5}, 3 d^{4} 4 s$, and $3 d^{3}$ $4 s^{2}$. When the ion is excited these may be transformed into the odd configurations $3 d^{4} 4 p$ and $3 d^{3}$ $4 s 4 p$. Further excitation will produce, alternately, higher even and odd configurations whose terms form series with those from the lower configurations according to their parity. The terms belonging to these electron configurations, according to Hund's theory, are listed in table 5, and are valid for all spectra of the $\mathrm{V}$ I isoelectronic sequence. A similar but more extensive table in which the theoretical terms are coordinated with their limits is given as taple 22 on page XXXVIII of the Introduction to volume 1 of "Atomic energy levels", Circular 467 of the National Bureau of Standards. The terms actually found are indicated by bold-face type in table 5, and their values are given in tables 3 and 4. No terms from the configuration $3 d^{3} 4 s 4 p$ have as yet been found.

The correlation of the observed with the theoretical $\mathrm{Cr}$ II terms was achieved with the help of various guides. The terms of the $3 d^{5}$ configuration, and the doublets of $3 d^{4} 4 s$ that are derived from singlets, exhibit small separations, $\Delta \nu$, of only a few $\mathrm{cm}^{-1}$ between their component levels. With the quartet terms partial inversion occurs among the levels so that the algebraic sum of the $\Delta \nu$ 's differs from zero by only a few wave number units. Some of the doublets are also inverted. On the other hand, the terms from the $3 d^{4} 4 s$ and $3 d^{3} 4 s^{2}$ configurations have wide level separations ranging from some tens to several hundred $\mathrm{cm}^{-1}$. In some of the terms, however, the intervals are of the same order of magnitude for each of the configurations. This is particularly the case for terms with $L$-values greater than 2. In such cases the configuration assignments are those indicated by Laporte [15] for the $d^{5}$ configuration, by Laporte and Platt [16] for the $d^{4}$ and $d^{3}$ configurations, or by Racah [17] and coworkers, notably A. Schweitzer [18], in consideration of the interaction between the $d^{5}$ and $d^{4} s$ configurations.

It is to be noted that the terms assigned to $3 d^{3}$ $4 s^{2}$ lie very high and give only weak combinations with some of the $3 d^{4} 4 p$ terms. It is plausible that they could belong to the $3 d^{4} 4 s$ configuration, but their positions in the term scale argue in favor of the assigned configuration.

\subsection{Series and Ionization Potential}

Among the terms of table 3 there are eight pairs that belong to Rydberg sequences. These are given in table 6. It is well known that limits calculated with a Rydberg formula for 2-member series are only approximately correct and do not give the best value for the ionization potential. In 1927 Russell [19] stated: "Experience shows that this value is usually nearly correct when the series is produced by the removal of an $s$ electron, since in such series the Ritz correction is almost always small. Series involving changes in a $d$ electron are usually very regular, except for the lowest term, when this involves the binding of the electron as part of an incomplete shell. In this case the energy of binding is considerably increased, and the application of a single Rydberg formula puts the limit a great deal too high". For this reason the five pairs of terms listed in table 6 as due to the migration of a $d$-electron have not been used to determine the ionization potential of $\mathrm{Cr}^{+}$. Their representation by a Rydberg formula leads to rather erratic values for the series limit. On the other hand, the three pairs of terms due to the $n s$-electrons give the values $135,400,134,500$, and $137,500 \mathrm{~cm}^{-1}$, respectively, for the distance between ${ }^{6} \mathrm{~S}_{21 / 2}$ of $\mathrm{Cr}$ II and ${ }^{5} \mathrm{D}_{0}$ of Cr III. The mean of these is only slightly less than $136,000 \mathrm{~cm}^{-1}$, the value adopted tentatively for the ground state of Cr III. But evidence derived from other spectra in which series of three or more members are known indicates that this value also is too high.

To arrive at the true series limit, from which a correct value for the ionization potential may be derived, it is therefore necessary to apply corrections to the denominators of the Rydberg formula. These corrections can be determined accurately only in spectra in which three or more series members are represented by a Ritz formula, as illustrated by Shenstone [20], for example, in Cu II. For spectra with only two series members Russell [21] has shown, recently, that very close estimates of the Ritz corrections may be found by comparisons with spectra of neighboring elements. Thus, for $\mathrm{Cr}$ in the limit of $136,000 \mathrm{~cm}^{-1}$ given by the Rydberg formula is found to require a Ritz correction of $-2,940 \mathrm{~cm}^{-1}$, which gives $133,060 \mathrm{~cm}^{-1}$ for the distance between $a^{6} \mathrm{~S}_{21 / 2}$ of $\mathrm{Cr}$ II and $a^{5} \mathrm{D}_{0}$ of Cr III. This corresponds to an ionization potential of $16.49 \mathrm{v}$.

The work described would not have reached its present status without the friendly cooperation of various individuals. H. D. Babcock, of the Mt. Wilson Observatory, made available to me his unpublished Zeeman patterns of the chromium spectra. A. S. King, also of the Mt. Wilson Observatory, placed at my disposal his furnace observations of the ultraviolet spectra, in advance of publication, and some of his arc and spark spectrograms of the chromium spectra. Likewise, R. J. Lang, University of Alberta, sent me his unpublished list of chromium wavelengths in the far ultraviolet. Both G. R. Harrison, Massachusetts Institute of Technology, and W. F. Meggers, National Bureau of Standards, secured Zeeman effect observations with the high fields and high dispersion of the MIT magnet and spectrographs. Finally, W. H. Seaquist, B. H. Monish, and W. F. Hausstein of the Bureau gave valuable advice and suggestions on the design and construction of the enclosed spark lamp. It is a pleasure for me to express to each of them my appreciation for his contribution to this work. 
TABLE 1. Wavelengths of $\mathrm{Cr}$ II in air

\begin{tabular}{|c|c|c|c|c|}
\hline Wavelength & Intensity & Wave No. & Term combination & Zeeman effect \\
\hline $\begin{array}{l}7311.60 \\
6418.87 \\
6415.60 \\
6358.06 \\
6305.61\end{array}$ & $\begin{array}{r}2 \\
20 \\
2 \\
1 \\
15\end{array}$ & $\begin{array}{l}13673.14 \\
15574.77 \\
15582.71 \\
15723.73 \\
15854.52\end{array}$ & $\begin{array}{l}c^{4} \mathrm{D}_{31 / 2}-z^{4} \mathrm{~F}_{41 / 2}^{\circ} \\
c^{4} \mathrm{~F}_{41 / 2}-x^{4} \mathrm{~F}_{41 / 2}^{\circ} \\
c^{4} \mathrm{~F}_{41 / 2}-x^{4} \mathrm{~F}_{31 / 2}^{\circ} \\
b^{2} \mathbf{P}_{11 / 2}-y^{2} \mathbf{P}_{01 / 2}^{0} \\
b^{2} \mathbf{P}_{11 / 2}^{0}-y^{2} \mathbf{P}_{11 / 2}^{0}\end{array}$ & $\begin{array}{l}(0.00) 1.33 \dagger \\
(0.00) 1.36 \dagger\end{array}$ \\
\hline $\begin{array}{l}6282.92 \\
6274.93 \\
6271.84 \\
6263.22 \\
6239.78\end{array}$ & $\begin{array}{r}6 \\
2 \\
15 \\
6 \\
10\end{array}$ & $\begin{array}{l}15911.77 \\
15932.03 \\
15939.88 \\
15961.82 \\
16021.78\end{array}$ & $\begin{array}{l}c^{4} \mathrm{~F}_{31 / 2}-x^{4} \mathrm{~F}_{21 / 2}^{\circ} \\
c^{4} \mathrm{~F}_{31 / 2}^{\circ}-x^{4} \mathrm{~F}_{41 / 2}^{\circ 1} \\
c^{4} \mathrm{~F}_{31 / 2}-x^{4} \mathrm{~F}_{31 / 2}^{\circ} \\
d^{2} \mathrm{~F}_{21 / 2}-z^{2} \mathrm{D}_{11 / 2}^{\circ} \\
c^{4} \mathrm{D}_{01 / 2}^{\circ}-z^{4} \mathrm{D}_{01 / 2}^{\circ}\end{array}$ & $(0.00) 1.24 \dagger$ \\
\hline $\begin{array}{l}6226.67 \\
6208.20 \\
6195.17 \\
6181.38 \\
6179.17\end{array}$ & $\begin{array}{r}8 \\
2 \\
15 \\
5 \\
35\end{array}$ & $\begin{array}{l}16055.52 \\
16103.28 \\
16137.15 \\
16173.15 \\
16178.94\end{array}$ & $\begin{array}{l}c^{4} \mathrm{D}_{11 / 2}-z^{4} \mathrm{D}_{01 / 2}^{\circ}{ }^{\circ} \\
c^{4} \mathrm{D}_{01 / 2}-z^{4} \mathrm{D}_{11 / 2}^{\circ} \\
c^{4} \mathrm{D}_{11 / 2}-z^{4} \mathrm{D}_{11 / 2}^{\circ} \\
e^{2} \mathrm{G}_{41 / 2}-y^{2} \mathrm{~F}_{31 / 2}^{\circ} \\
d^{2} \mathrm{G}_{31 / 2}-z^{2} \mathrm{H}_{41 / 2}^{\circ}\end{array}$ & $(0.00) 0.95 \dagger$ \\
\hline $\begin{array}{l}6176.95 \\
6168.46 \\
6157.80 \\
6147.17 \\
6138.77\end{array}$ & $\begin{array}{r}15 \\
2 \\
7 \\
20 \\
10\end{array}$ & $\begin{array}{l}16184.75 \\
16207.03 \\
16235.08 \\
16263.16 \\
16285.41\end{array}$ & $\begin{array}{l}c^{4} \mathrm{D}_{21 / 2}-z^{4} \mathrm{D}_{11 / 2}^{\circ} \\
c^{4} \mathrm{~F}_{21 / 2}-x^{4} \mathrm{~F}_{21 / 2}^{2} \\
c^{4} \mathrm{~F}_{21 / 2}-x^{4} \mathrm{~F}_{31 / 2}^{\circ} \\
c^{4} \mathrm{D}_{11 / 2}-z^{4} \mathrm{D}_{21 / 2}^{\circ} \\
d^{2} \mathrm{G}_{31 / 2}-z^{2} \mathrm{~F}_{21 / 2}^{\circ}\end{array}$ & $(0.00) 0.94 \dagger$ \\
\hline $\begin{array}{l}\text { 6134. } 38 \\
6129.23 \\
6116.42 \\
6112.27\end{array}$ & $\begin{array}{r}2 \\
18 \\
2 \\
3\end{array}$ & $\begin{array}{l}\text { 16297. } 06 \\
16310.76 \\
16344.92 \\
16356.02\end{array}$ & $\begin{array}{l}c^{4} \mathrm{~F}_{11 / 2}-x^{4} \mathrm{~F}_{11 / 2}^{\circ} \\
c^{4} \mathrm{D}_{21 / 2}-z^{4} \mathrm{D}_{21 / 2}^{\circ} \\
d^{2} \mathrm{~F}_{31 / 2}-z^{2} \mathrm{D}_{21 / 2}^{\circ} \\
c^{4} \mathrm{D}_{31 / 2}-z^{4} \mathrm{D}_{21 / 2}^{\circ}\end{array}$ & $(0.00) 1.38 \dagger$ \\
\hline $\begin{array}{l}6089.69 \\
6081.52 \\
6070.10 \\
6069.69 \\
6067.99\end{array}$ & $\begin{array}{r}15 \\
3 \\
2 \\
1 \\
7\end{array}$ & $\begin{array}{l}16416.66 \\
16438.72 \\
16469.64 \\
16470.76 \\
16475.37\end{array}$ & $\begin{array}{l}d^{2} \mathrm{G}_{41 / 2}-z^{2} \mathrm{H}_{51 / 2}^{\circ} \\
d^{2} \mathrm{G}_{41 / 2}-z^{2} \mathrm{~F}_{31 / 2}^{\circ} \\
c^{4} \mathrm{D}_{21 / 2}-z^{4} \mathrm{D}_{31 / 2}^{\circ} \\
c^{4} \mathrm{~F}_{41 / 2}-y^{2} \mathrm{H}_{41 / 2}^{\circ} \\
c^{4} \mathrm{~F}_{41 / 2}-y^{2} \mathrm{H}_{51 / 2}^{\circ}\end{array}$ & $(0.00) 1.07 \dagger$ \\
\hline $\begin{array}{l}6053.48 \\
6040.57 \\
5913.86 \\
5895.89 \\
5841.88\end{array}$ & $\begin{array}{l}75 \\
10 \\
12 \\
15 \\
12\end{array}$ & $\begin{array}{l}16514.86 \\
16550.16 \\
16904.76 \\
16956.28 \\
17113.04\end{array}$ & $\begin{array}{l}c^{4} \mathrm{D}_{31 / 2}-z^{4} \mathrm{D}_{31 / 2}^{\circ} \\
c^{4} \mathrm{~F}_{41 / 2}-x^{4} \mathrm{G}_{51 / 2}^{\circ} \\
c^{4} \mathrm{~F}_{31 / 2}^{\circ}-x^{4} \mathrm{G}_{41 / 2}^{\circ 1}\end{array}$ & $(0.00) 1.42 \dagger$ \\
\hline $\begin{array}{l}\text { 5827. } 24 \\
5795.28 \\
5790.29 \\
5775.81 \\
5685.90\end{array}$ & $\begin{array}{r}12 \\
2 \\
20 \\
4 \\
7\end{array}$ & $\begin{array}{l}17156.04 \\
17250.65 \\
17265.78 \\
17308.80 \\
17582.50\end{array}$ & $\begin{array}{l}c^{4} \mathrm{~F}_{21 / 2}-x^{4} \mathrm{G}_{31 / 2}^{\circ} \\
c^{4} \mathrm{~F}_{11 / 2}-x^{4} \mathrm{G}_{21 / 2}^{\circ} \\
e^{2} \mathrm{D}_{21 / 2}-w^{2} \mathrm{~F}_{31 / 2}^{\circ} \\
d^{2} \mathrm{G}_{41 / 2}-y^{2} \mathrm{G}_{31 / 2}^{\circ}\end{array}$ & \\
\hline $\begin{array}{l}5678.44 \\
5620.68 \\
5613.18 \\
5542.48 \\
5510.71\end{array}$ & $\begin{array}{r}20 \\
30 \\
10 \\
3 \\
20\end{array}$ & $\begin{array}{l}17605.60 \\
17786.52 \\
17810.28 \\
18037.47 \\
18141.46\end{array}$ & $\begin{array}{l}d^{2} \mathrm{G}_{31 / 2}-y^{2} \mathrm{G}_{31 / 2}^{\circ} \\
d^{2} \mathrm{G}_{41 / 2}-y^{2} \mathrm{G}_{41 / 2}^{\circ} \\
d^{2} \mathrm{G}_{31 / 2}-y^{2} \mathrm{G}_{41 / 2}^{\circ} \\
b^{4} \mathrm{P}_{21 / 2}-z^{4} \mathrm{P}_{11 / 2}^{\circ}\end{array}$ & $\begin{array}{l}(0.00) 0.92 \dagger \\
(0.00) 1.10 \dagger \\
(0.00 w) 1.42 A \dagger\end{array}$ \\
\hline $\begin{array}{l}5508.63 \\
5503.21 \\
5502.07 \\
5497.80 \\
5478.37\end{array}$ & $\begin{array}{r}30 \\
25 \\
40 \\
3 \\
50\end{array}$ & $\begin{array}{l}18148.30 \\
18166.18 \\
18169.94 \\
18184.06 \\
18248.55\end{array}$ & $\begin{array}{l}b^{4} \mathrm{G}_{31 / 2}-z^{4} \mathrm{~F}_{21 / 2}^{\circ} \\
b^{4} \mathrm{G}_{21 / 2}-z^{4} \mathrm{~F}_{11 / 2}^{\circ} \\
b^{4} \mathrm{G}_{41 / 2}-z^{4} \mathrm{~F}_{31 / 2}^{\circ} \\
b^{4} \mathrm{P}_{11 / 2}-z^{6} \mathrm{P}_{21 / 2}^{\circ} \\
b^{4} \mathrm{G}_{51 / 2}^{\circ}-z^{4} \mathrm{~F}_{41 / 2}^{\circ}\end{array}$ & $\begin{array}{l}(0.00) 0.95 \\
(0.00) 0.78 \\
(0.00) 1.03 A \dagger \\
(0.00 w) 1.10 A \dagger\end{array}$ \\
\hline $\begin{array}{l}5477.80 \\
5477.49 \\
5472.60 \\
5455.86 \\
5446.77\end{array}$ & $\begin{array}{r}2 \\
10 \\
12 \\
8 \\
10\end{array}$ & $\begin{array}{l}18250.45 \\
18251.48 \\
18267.79 \\
18323.84 \\
18354.42\end{array}$ & $\begin{array}{l}e^{2} \mathrm{D}_{11 / 2}-x^{2} \mathrm{P}_{01 / 2}^{\circ} \\
b^{4} \mathrm{G}_{21 / 2}-z^{4} \mathrm{~F}_{21 / 2}^{\circ} \\
b^{4} \mathrm{G}_{31 / 2}-z^{4} \mathrm{~F}_{31 / 2}^{\circ} \\
b^{4} \mathrm{G}_{41 / 2}-z^{4} \mathrm{~F}_{41 / 2}^{\circ} \\
c^{4} \mathrm{P}_{11 / 2}^{\circ}-w^{4} \mathrm{D}_{21 / 2}^{\circ}\end{array}$ & \\
\hline $\begin{array}{l}5420.91 \\
5419.38 \\
5414.86 \\
5407.62 \\
5379.80\end{array}$ & $\begin{array}{r}25 \\
2 \\
7 \\
25 \\
6\end{array}$ & $\begin{array}{l}18441.97 \\
18447.18 \\
18462.58 \\
18487.30 \\
18582.90\end{array}$ & $\begin{array}{l}b^{4} \mathrm{P}_{11 / 2}-z^{4} \mathrm{P}_{01 / 2}^{\circ} \\
b^{4} \mathrm{P}_{01 / 2}-z^{6} \mathrm{P}_{11 / 2}^{0} \\
c^{4} \mathrm{P}_{21 / 2}-w^{4} \mathrm{P}_{31 / 3}^{\circ} \\
b^{4} \mathrm{P}_{21 / 2}-z^{4} \mathrm{P}_{21 / 2}^{0} \\
b^{2} \mathrm{P}_{11 / 2}-x^{2} \mathrm{P}_{11 / 2}^{0}\end{array}$ & $\begin{array}{l}(0.56) 1.16,2.26 \dagger \\
(0.00) 1.66 \dagger\end{array}$ \\
\hline
\end{tabular}


TABLE 1. Wavelengths of $\mathrm{Cr}$ II in air-Continued

\begin{tabular}{|c|c|c|c|c|}
\hline Wavelength & Intensity & Wave No. & Term combination & Zeeman effect \\
\hline $\begin{array}{l}5369.36 \\
5368.09 \\
5354.72 \\
5346.54 \\
5337.81\end{array}$ & $\begin{array}{r}15 \\
7 \\
3 \\
5 \\
30\end{array}$ & $\begin{array}{l}\text { 18619. } 03 \\
18623.43 \\
18669.93 \\
18698.48 \\
18729.08\end{array}$ & $\begin{array}{l}a^{4} \mathrm{~F}_{41 / 2}-z^{6} \mathrm{D}_{41 / 2}^{\circ} \\
a^{4} \mathrm{~F}_{11 / 2}-z^{6} \mathrm{D}_{21 / 2}^{\circ} \\
a^{4} \mathrm{~F}_{31 / 2}-z^{6} \mathrm{D}_{41 / 2}^{\circ} \\
b^{4} \mathrm{P}_{11 / 2}^{\circ}-z^{4} \mathrm{P}_{11 / 2}^{\circ} \\
b^{4} \mathrm{~F}_{21 / 2}-z^{4} \mathrm{~F}_{11 / 2}^{111}\end{array}$ & $(0.38) 0.74,1.34, \mathbf{1 . 9 6} \dagger$ \\
\hline $\begin{array}{l}\text { 5334. } 89 \\
5318.38 \\
5316.28 \\
5313.61 \\
5310.73\end{array}$ & $\begin{array}{r}50 \\
15 \\
7 \\
40 \\
30\end{array}$ & $\begin{array}{l}\text { 18739. } 33 \\
18797.50 \\
18804.93 \\
18814.38 \\
18824.58\end{array}$ & $\begin{array}{l}b^{4} \mathrm{~F}_{11 / 2}-z^{4} \mathrm{~F}_{11 / 2}^{\circ} \\
b^{4} \mathrm{P}_{01 / 2}-z^{4} \mathrm{P}_{01 / 2}^{\circ} \\
b^{2} \mathrm{P}_{11 / 2}-x^{2} \mathrm{D}_{21 / 2}^{\circ} \\
b^{4} \mathrm{~F}_{21 / 2}-z^{4} \mathrm{~F}_{21 / 2}^{\circ} \\
b^{4} \mathrm{~F}_{11 / 2}-z^{4} \mathrm{~F}_{21 / 2}^{\circ}\end{array}$ & $\begin{array}{l}(0.00) 0.42 \dagger \\
(0.00) 1.06 \dagger \\
(0.27,0.88) 0.80,1.41,2.02 \dagger\end{array}$ \\
\hline $\begin{array}{l}5308.46 \\
5305.86 \\
5280.08 \\
5279.88 \\
5274.99\end{array}$ & $\begin{array}{l}35 \\
40 \\
30 \\
50 \\
75\end{array}$ & $\begin{array}{l}\text { 18832. } 63 \\
18841.85 \\
18933.85 \\
18934.57 \\
18952.12\end{array}$ & $\begin{array}{l}b^{4} \mathbf{F}_{31 / 2}-z^{4} \mathbf{F}_{21 / 2}^{\circ} \\
b^{4} \mathbf{P}_{21 / 2}-z^{6} \mathrm{D}_{21 / 2}^{\circ} \\
b^{4} \mathbf{F}_{21 / 2}-z^{4} \mathbf{F}_{31 / 2}^{\circ} \\
b^{4} \mathbf{F}_{41 / 2}-z^{4} \mathbf{F}_{31 / 2}^{\circ} \\
b^{4} \mathbf{F}_{31 / 2}^{\circ}-z^{4} \mathbf{F}_{31 / 2}^{\circ}\end{array}$ & $\begin{array}{l}(0.00 W) 1.72 B \dagger \\
(0.00 w) 1.66 \dagger \\
(0.00) 1.35 \\
(0.00 d ?) 1.53 B \dagger \\
(0.00) 1.28 \dagger\end{array}$ \\
\hline $\begin{array}{l}5249.43 \\
5246.76 \\
5237.35 \\
5232.54 \\
5210.87\end{array}$ & $\begin{array}{r}20 \\
30 \\
100 \\
20 \\
10\end{array}$ & $\begin{array}{l}\text { 19044. } 40 \\
19054.09 \\
19088.33 \\
19105.87 \\
19185.33\end{array}$ & $\begin{array}{l}b^{4} \mathrm{P}_{11 / 2}-z^{4} \mathrm{P}_{21 / 2}^{\circ} \\
b^{4} \mathrm{P}_{01 / 2}-z^{4} \mathrm{P}_{11 / 2}^{\circ} \\
b^{4} \mathbf{F}_{41 / 2}-z^{4} \mathbf{F}_{41 / 2}^{\circ} \\
b^{4} \mathbf{F}_{31 / 2}-z^{4} \mathbf{F}_{41 / 2}^{\circ} \\
b^{4} \mathrm{P}_{11 / 2}-z^{6} \mathrm{D}_{01 / 2}^{\circ}\end{array}$ & $\begin{array}{l}(0.43) 1.41,2.26 \dagger \\
(0.00) 1.34 \dagger \\
(0.00) 1.61 w \dagger \\
(0.68) 0.93,2.52 \dagger\end{array}$ \\
\hline $\begin{array}{l}5202.60 \\
5191.44 \\
5188.91 \\
5186.12 \\
5153.50\end{array}$ & $\begin{array}{r}3 \\
2 \\
1 \\
2 \\
20\end{array}$ & $\begin{array}{l}19215.82 \\
19257.13 \\
19266.52 \\
19276.88 \\
19398.90\end{array}$ & $\begin{array}{r}d^{2} \mathrm{~F}_{21 / 2}-y^{2} \mathrm{G}_{31 / 2}^{0} \\
b^{4} \mathrm{P}_{11 / 2}-z^{6} \mathrm{D}_{11 / 2}^{\circ} \\
d^{2} \mathrm{~F}_{21 / 2}-y^{2} \mathrm{D}_{21 / 2}^{\circ} \\
d^{2} \mathrm{D}_{11 / 2}-z^{2} \mathrm{D}_{11 / 2}^{\circ} \\
b^{4} \mathrm{P}_{11 / 2}-z^{6} \mathrm{D}_{21 / 2}^{0}\end{array}$ & \\
\hline $\begin{array}{l}5142.52 \\
5137.09 \\
5121.10 \\
5116.07 \\
5110.45\end{array}$ & $\begin{array}{l}2 \\
7 \\
2 \\
2 \\
2\end{array}$ & $\begin{array}{l}19440.32 \\
19460.87 \\
19521.63 \\
19540.82 \\
19562.31\end{array}$ & $\begin{array}{l}d^{2} \mathbf{F}_{31 / 2}-y^{2} \mathrm{G}_{41 / 2}^{\circ} \\
c^{4} \mathbf{P}_{21 / 2}-x^{4} \mathbf{P}_{21 / 2}^{\circ} \\
b^{4} \mathbf{P}_{01 / 2}-z^{6} \mathrm{D}_{01 / 2}^{\circ} \\
c^{4} \mathbf{F}_{41 / 2}-w^{4} \mathrm{D}_{31 / 2}^{\circ 1}\end{array}$ & $(0.00) 1.64 \dagger$ \\
\hline $\begin{array}{l}5097.33 \\
5091.14 \\
5090.36 \\
5085.70 \\
5076.16\end{array}$ & $\begin{array}{l}7 \\
2 \\
1 \\
4 \\
4\end{array}$ & $\begin{array}{l}19612.66 \\
19636.51 \\
19639.52 \\
19657.51 \\
19694.46\end{array}$ & $\begin{array}{l}b^{4} \mathrm{P}_{01 / 2}-z^{6} \mathrm{D}_{11 / 2}^{\circ} \\
c^{4} \mathrm{P}_{11 / 2}-x^{4} \mathrm{P}_{11 / 2}^{\circ} \\
d^{2} \mathrm{D}_{11 / 2}-z^{2} \mathrm{D}_{21 / 2}^{\circ} \\
d^{2} \mathrm{D}_{21 / 2}-z^{2} \mathrm{D}_{21 / 2}^{\circ} \\
c^{4} \mathrm{P}_{21 / 2}-x{ }^{4} \mathrm{P}_{11 / 2}^{\circ}\end{array}$ & (?) $1.51,2.20 \dagger$ \\
\hline $\begin{array}{l}5031.28 \\
5024.52 \\
4952.79 \\
4912.50 \\
4901.66\end{array}$ & $\begin{array}{r}3 \\
10 \\
12 \\
15 \\
15\end{array}$ & $\begin{array}{l}19870.13 \\
19896.87 \\
20185.06 \\
20350.57 \\
20395.58\end{array}$ & $\begin{array}{l}c^{4} \mathbf{F}_{31 / 2}-w^{4} \mathrm{D}_{21 / 2}^{\circ} \\
d^{2} \mathbf{F}_{21 / 2}-y^{2} \mathbf{F}_{21 / 2}^{\circ} \\
d^{2} \mathbf{F}_{31 / 2}-y^{2} \mathbf{F}_{31 / 2}^{\circ} \\
d^{2} \mathbf{G}_{31 / 2}-x^{2} \mathrm{G}_{31 / 2}^{\circ} \\
d^{2} \mathbf{G}_{41 / 2}-x^{2} \mathbf{G}_{41 / 2}^{\circ}\end{array}$ & $\begin{array}{l}(0.00) 0.86 \dagger \\
(0.00) 1.19 \dagger \\
(0.00) 0.91 \dagger \\
(0.00) 1.15 \dagger\end{array}$ \\
\hline $\begin{array}{l}4884.58 \\
4876.41 \\
4876.37 \\
4864.31 \\
4860.20\end{array}$ & $\begin{array}{l}12 \\
20 \\
40 \\
60 \\
20\end{array}$ & $\begin{array}{l}\text { 20466. } 89 \\
20501.18 \\
20501.49 \\
20552.18 \\
20569.56\end{array}$ & $\begin{array}{l}a^{4} \mathbf{F}_{21 / 2}-z^{4} \mathbf{F}_{11 / 2}^{\circ} \\
a^{4} \mathbf{F}_{31 / 2}-z^{4} \mathbf{F}_{21 / 2}^{\circ} \\
a^{4} \mathbf{F}_{11 / 2}-z^{4} \mathbf{F}_{11 / 2}^{\circ} \\
a^{4} \mathbf{F}_{21 / 2}-z^{4} \mathbf{F}_{21 / 2}^{\circ} \\
a^{4} \mathbf{F}_{41 / 2}-z^{4} \mathbf{F}_{31 / 2}^{\circ}\end{array}$ & $\begin{array}{l}(. .2) 0.12,0.73,1.39,2.05 \\
(0.00) 1.64 B \dagger \\
(0.00) 0.41 \\
(0.00) 1.04 \\
(0.00) 1.59 B\end{array}$ \\
\hline $\begin{array}{l}\text { 4857. } 60 \\
\text { 4856. } 18 \\
4848.24 \\
4836.22 \\
4832.38\end{array}$ & $\begin{array}{r}2 \\
20 \\
75 \\
25 \\
3\end{array}$ & $\begin{array}{l}20580.57 \\
20586.58 \\
20620.30 \\
20671.55 \\
20687.97\end{array}$ & $\begin{array}{l}c^{4} \mathbf{F}_{41 / 2}-w^{4} \mathbf{F}_{41 / 2}^{\circ} \\
a^{4} \mathbf{F}_{11 / 2}-z^{4} \mathbf{F}_{21 / 2}^{\circ} \\
a^{4} \mathbf{F}_{31 / 2}-z^{4} \mathbf{F}_{31 / 2}^{\circ} \\
a^{4} \mathbf{F}_{21 / 2}-z^{4} \mathbf{F}_{31 / 2}^{\circ}\end{array}$ & $\begin{array}{l}\text { (?) } 0.74,1.36,2.04 \dagger \\
(0.00) 1.25 \\
(?) 1.71 B \dagger\end{array}$ \\
\hline $\begin{array}{l}\text { 4824. } 12 \\
\text { 4815. } 29 \\
\text { 4812. } 34 \\
\text { 4794. } 79 \\
\text { 4793. } 09\end{array}$ & $\begin{array}{r}100 \\
1 \\
25 \\
1 \\
2\end{array}$ & $\begin{array}{l}\text { 20723. } 40 \\
20761.40 \\
20774.12 \\
20850.16 \\
20857.56\end{array}$ & $\begin{array}{l}a^{4} \mathbf{F}_{41 / 2}-z^{4} \mathrm{~F}_{41 / 2}^{\circ} \\
e^{2} \mathrm{D}_{11 / 2}-w^{2} \mathrm{D}_{11 / 2}^{\circ} \\
a^{4} \mathbf{F}_{31 / 2}-z^{4} \mathbf{F}_{41 / 2}^{1} \\
e^{2} \mathrm{D}_{21 / 2}-w^{2} \mathrm{D}_{21 / 2}^{\circ} \\
c^{4} \mathbf{F}_{31 / 2}-w^{4} \mathbf{F}_{31 / 2}^{\circ}\end{array}$ & $\begin{array}{l}(0.00) 1.34 \\
(0.00) 1.63 B \dagger\end{array}$ \\
\hline $\begin{array}{l}4765.06 \\
4761.40 \\
4749.75 \\
4715.12 \\
4713.27\end{array}$ & $\begin{array}{l}2 \\
2 \\
1 \\
3 \\
1\end{array}$ & $\begin{array}{l}\text { 20980. } 25 \\
20996.37 \\
21047.87 \\
21202.45 \\
21210.78\end{array}$ & $\begin{array}{l}c^{2} \mathrm{D}_{11 / 2}-z^{2} \mathrm{D}_{11 / 2}^{\circ} \\
c^{2} \mathrm{D}_{21 / 2}-y^{4} \mathrm{P}_{21 / 2}^{\circ} \\
c^{4} \mathrm{~F}_{21 / 2}-w^{4} \mathrm{~F}_{21 / 2}^{\circ} \\
c^{2} \mathrm{D}_{11 / 2}-z^{2} \mathbf{P}_{01 / 2}^{\circ} \\
d^{2} \mathrm{D}_{11 / 2}-z^{2} \mathbf{F}_{21 / 2}^{\circ}\end{array}$ & \\
\hline
\end{tabular}


TABLE 1. Wavelengths of $\mathrm{Cr}$ II in air-Continued

\begin{tabular}{|c|c|c|c|c|}
\hline Wavelength & Intensity & Wave No. & Term combination & Zeeman effect \\
\hline $\begin{array}{l}\text { 4710. } 78 \\
4697.61 \\
4684.78 \\
4634.10 \\
4618.82\end{array}$ & $\begin{array}{r}1 \\
3 \\
2 \\
40 \\
50\end{array}$ & $\begin{array}{l}\text { 21221. } 99 \\
21281.48 \\
21339.76 \\
21573.14 \\
21644.51\end{array}$ & $\begin{array}{c}c^{4} \mathrm{~F}_{11 / 2}-w^{4} \mathrm{~F}_{11 / 2}^{\circ} \\
c^{2} \mathrm{D}_{21 / 2}-z^{2} \mathrm{D}_{21 / 2}^{\circ} \\
c^{2} \mathrm{D}_{21 / 2}-z^{2} \mathrm{P}_{11 / 2}^{\circ} \\
b^{4} \mathrm{~F}_{11 / 2}-z^{4} \mathrm{D}_{01 / 2}^{\circ} \\
b^{4} \mathrm{~F}_{21 / 2}-z^{4} \mathrm{D}_{11 / 2}^{\circ}\end{array}$ & $\begin{array}{l}(0.24) 0.24,0.62 \dagger \\
(0.00) 0.82 A \dagger\end{array}$ \\
\hline $\begin{array}{l}4616.64 \\
4592.07 \\
4589.89 \\
4588.22 \\
4587.30\end{array}$ & $\begin{array}{r}25 \\
25 \\
4 \\
75 \\
2\end{array}$ & $\begin{array}{l}21654.73 \\
21770.59 \\
21780.93 \\
21788.86 \\
21793.23\end{array}$ & $\begin{array}{l}b^{4} \mathrm{~F}_{11 / 2}-z^{4} \mathrm{D}_{11 / 2}^{\circ} \\
b^{4} \mathrm{~F}_{21 / 2}^{\circ}-z^{4} \mathrm{D}_{21 / 2}^{\circ} \\
b^{4} \mathrm{~F}_{11 / 2}-z^{4} \mathrm{D}_{21 / 2}^{\circ} \\
b^{4} \mathrm{~F}_{31 / 2}-z^{4} \mathrm{D}_{21 / 2}^{\circ} \\
d^{2} \mathrm{G}_{41 / 2}^{\circ}-x^{2} \mathrm{~F}_{31 / 2}^{\circ}\end{array}$ & $\begin{array}{l}(1.19) \dot{0}, 0.79,1.62 \dagger \\
(0.50, \mathbf{0} 6) 0.41,0.82, \mathbf{1 . 2 6}, 1.60,1.90 \dagger \\
(0.00,0.18,0.35) \mathbf{0 . 9 0}, 1.09,1.28,1.48\end{array}$ \\
\hline $\begin{array}{l}4572.84 \\
4565.77 \\
4564.27 \\
4558.66 \\
4555.01\end{array}$ & $\begin{array}{r}2 \\
10 \\
2 \\
100 \\
30\end{array}$ & $\begin{array}{l}21862.14 \\
21895.99 \\
21903.19 \\
21930.14 \\
21947.72\end{array}$ & $\begin{array}{l}e^{2} \mathrm{D}_{21 / 2}-v^{2} \mathrm{~F}_{31 / 2}^{\circ} \\
a^{2} \mathrm{~F}_{21 / 2}-z^{4} \mathrm{D}_{11 / 2}^{\circ} \\
b^{4} \mathrm{~F}_{41 / 2}-z^{4} \mathrm{D}_{31 / 2}^{\circ} \\
b^{4} \mathrm{~F}_{31 / 2}-z^{4} \mathrm{D}_{31 / 2}^{\circ}\end{array}$ & $\begin{array}{l}(0.00) 0.51,0.78 \\
(0.00) 1.07 A \dagger \\
(0.66) 1.33 w^{\dagger}\end{array}$ \\
\hline $\begin{array}{l}4546.63 \\
4539.61 \\
4515.83 \\
4489.14 \\
4465.77\end{array}$ & $\begin{array}{l}2 \\
3 \\
4 \\
2 \\
5\end{array}$ & $\begin{array}{l}\text { 21988. } 17 \\
22022.17 \\
22138.14 \\
22269.76 \\
22386.29\end{array}$ & $\begin{array}{l}a^{2} \mathrm{~F}_{21 / 2}-z^{4} \mathrm{D}_{21 / 2}^{\circ} \\
d^{2} \mathrm{G}_{31 / 2}-x^{2} \mathrm{H}_{21 / 2}^{\circ} \\
a^{2} \mathrm{~F}_{31 / 2}-z^{4} \mathrm{D}_{21 / 2}^{\circ} \\
d^{2} \mathrm{G}_{41 / 2}-x^{2} \mathrm{H}_{51 / 2}^{\circ}\end{array}$ & \\
\hline $\begin{array}{l}4362.93 \\
4341.09 \\
4308.81 \\
4306.95 \\
4284.21\end{array}$ & $\begin{array}{r}3 \\
10 \\
3 \\
10 \\
20\end{array}$ & $\begin{array}{l}\text { 22913. } 96 \\
\text { 23029. } 24 \\
23201.76 \\
23211.78 \\
23334.98\end{array}$ & $\begin{array}{l}c^{2} \mathrm{D}_{11 / 2}-z^{2} \mathrm{~F}_{21 / 2}^{\circ} \\
c^{2} \mathrm{D}_{21 / 2}-z^{2} \mathrm{~F}_{31 / 2}^{\circ} \\
d^{2} \mathrm{D}_{11 / 2}-y^{2} \mathrm{~F}_{21 / 2}^{\circ} \\
a^{4} \mathrm{~F}_{11 / 2}-z^{4} \mathrm{D}_{01 / 2}^{\circ}\end{array}$ & $\begin{array}{l}(0.00) 1.13 \dagger \\
(0.00) 0.90 \dagger \\
(0.20) 0.22,0.63 \dagger\end{array}$ \\
\hline $\begin{array}{l}4278.11 \\
4275.58 \\
4269.29 \\
4268.96 \\
4265.05\end{array}$ & $\begin{array}{l}3 s \\
30 \\
10 \\
3 \\
2\end{array}$ & $\begin{array}{l}\text { 23368. } 26 \\
23382.08 \\
23416.53 \\
23418.34 \\
23439.81\end{array}$ & $\begin{array}{l}b^{2} \mathrm{D}_{11 / 2}-y{ }^{4} \mathrm{P}_{11 / 2}^{\circ} \\
a^{4} \mathrm{~F}_{21 / 2}-z^{4} \mathrm{D}_{11 / 2}^{\circ} \\
a^{4} \mathrm{~F}_{11 / 2}-z^{4} \mathrm{D}_{11 / 2}^{\circ} \\
d^{2} \mathrm{G}_{31 / 2}-w^{2} \mathrm{G}_{31 / 2}^{\circ}\end{array}$ & $\begin{array}{l}(0.00) 0.77 \\
(1.24) 0.00, \mathbf{0 . 8 0}, 1.62 \dagger\end{array}$ \\
\hline $\begin{array}{l}4261.92 \\
4256.17 \\
4252.63 \\
4246.41 \\
4245.12\end{array}$ & $\begin{array}{l}30 \\
5 l, d ? \\
10 \\
2 \\
2\end{array}$ & $\begin{array}{l}23457.02 \\
23488.71 \\
23508.27 \\
23542.70 \\
23549.85\end{array}$ & $\begin{array}{l}a^{4} \mathrm{~F}_{31 / 2}-z^{4} \mathrm{D}_{21 / 2}^{\circ} \\
d^{2} \mathrm{G}_{41 / 2}-w^{2} \mathrm{G}_{41 / 2}^{\circ} \\
a^{4} \mathrm{~F}_{21 / 2}-z^{4} \mathrm{D}_{21 / 2}^{\circ} \\
a^{4} \mathrm{~F}_{11 / 2}-z^{4} \mathrm{D}_{21 / 2}^{\circ}\end{array}$ & $\begin{array}{l}(0.00) 1.00 A \\
(0.00) 1.10 \\
(0.62, \mathbf{0 . 9 0} 0.56,0.90,1.23,1.62,1.89 \dagger\end{array}$ \\
\hline $\begin{array}{l}4242.36 \\
4233.26 \\
4229.81 \\
4227.73 \\
4224.85\end{array}$ & $\begin{array}{r}50 \\
18 \\
1 \\
1 \\
25\end{array}$ & $\begin{array}{l}23565.17 \\
23615.83 \\
23635.09 \\
23646.72 \\
23662.84\end{array}$ & $\begin{array}{c}a{ }^{4} \mathrm{~F}_{41 / 2}-z^{4} \mathrm{D}_{31 / 2}^{\circ} \\
a^{4} \mathrm{~F}_{31 / 2}-z^{4} \mathrm{D}^{31 / 2} \\
b^{4} \mathrm{P}_{21 / 2}-z^{4} \mathrm{D}_{11 / 2}^{\circ} \\
a^{2} \mathrm{~S}_{01 / 2}-y^{4} \mathrm{D}_{11 / 2}^{\circ} \\
b^{2} \mathrm{D}_{11 / 2}-z^{2} \mathrm{D}_{11 / 2}^{\circ}\end{array}$ & $\begin{array}{l}(0.00) 1.12 A \dagger \\
(0.63) 1.36 \dagger \\
(0.08) 0.86 \dagger\end{array}$ \\
\hline $\begin{array}{l}4222.00 \\
4217.07 \\
4215.76 \\
4209.05 \\
4207.36\end{array}$ & $\begin{array}{l}3 \\
2 \\
2 \\
3 \\
4\end{array}$ & $\begin{array}{l}23678.81 \\
23706.49 \\
23713.86 \\
23751.66 \\
23761.20\end{array}$ & $\begin{array}{l}c^{2} \mathrm{D}_{11 / 2}-x^{4} \mathrm{~F}_{11 / 2}^{\circ} \\
b^{4} \mathrm{D}_{11 / 2}-z^{4} \mathrm{P}_{01 / 2}^{\circ} \\
b^{4} \mathrm{D}_{01 / 2}-z^{4} \mathrm{P}_{0112}^{\circ} \\
b^{2} \mathrm{D}_{21 / 2}-z^{2} \mathrm{D}_{11 / 2}^{\circ} \\
b^{4} \mathrm{P}_{21 / 2}-z^{4} \mathrm{D}_{21 / 2}^{0}\end{array}$ & \\
\hline $\begin{array}{l}\text { 4195. } 41 \\
4195.33 \\
4181.50 \\
4179.43 \\
4172.60\end{array}$ & $\begin{array}{r}4 \\
6 \\
1 \\
12 \\
2\end{array}$ & $\begin{array}{l}23828.88 \\
23829.34 \\
23908.15 \\
23919.99 \\
23959.14\end{array}$ & $\begin{array}{c}b^{2} \mathrm{I}_{61 / 2}-z^{4} \mathrm{H}_{61 / 2}^{\circ} \\
b^{2} \mathrm{D}_{21 / 2}-y^{4} \mathbf{P}_{21 / 2}^{\circ} \\
c^{2} \mathrm{D}_{21 / 2}-y^{2} \mathrm{D}_{11 / 2}^{\circ} \\
b^{4} \mathrm{P}_{21 / 2}-z^{4} \mathrm{D}_{31 / 2}^{\circ} \\
b^{4} \mathrm{D}_{21 / 2}-z^{4} \mathrm{P}_{11 / 2}^{0}\end{array}$ & $\begin{array}{l}(0.79) 1.28 \dagger \\
(0.79) 1.56 \dagger\end{array}$ \\
\hline $\begin{array}{l}4171.92 \\
4170.86 \\
4170.65 \\
4161.07 \\
4151.00\end{array}$ & $\begin{array}{l}3 \\
1 \\
1 \\
2 \\
5\end{array}$ & $\begin{array}{l}23963.05 \\
23969.14 \\
23970.34 \\
24025.53 \\
24083.81\end{array}$ & $\begin{array}{l}b^{4} \mathrm{D}_{11 / 2}-z^{4} \mathrm{P}_{11 / 2}^{\circ} \\
c^{2} \mathrm{D}_{11 / 2}-y^{2} \mathrm{D}_{11 / 2}^{\circ} \\
b^{4} \mathrm{D}_{01 / 2}-z^{4} \mathrm{P}_{11 / 2}^{\circ} \\
b^{2} \mathrm{D}_{11 / 2}-z^{2} \mathrm{D}_{21 / 2}^{\circ} \\
b^{2} \mathrm{D}_{11 / 2}-z^{2} \mathrm{P}_{11 / 2}^{\circ}\end{array}$ & $(0.58) 0.64, \mathbf{0 . 9 2}, 1.25 \dagger$ \\
\hline $\begin{array}{l}\text { 4145. } 77 \\
4132.41 \\
4127.08 \\
4116.65 \\
4113.24\end{array}$ & $\begin{array}{r}25 \\
7 \\
4 \\
2 \\
5\end{array}$ & $\begin{array}{l}24114.20 \\
24192.16 \\
24223.40 \\
24284.77 \\
24304.90\end{array}$ & $\begin{array}{l}b^{2} \mathrm{D}_{21 / 2}-z^{2} \mathrm{D}_{21 / 2}^{\circ} \\
b^{4} \mathrm{P}_{11 / 2}-z^{4} \mathrm{D}_{11 / 2}^{\circ} \\
c^{2} \mathrm{D}_{21 / 2}-y^{2} \mathrm{D}_{21 / 2}^{\circ} \\
c^{2} \mathrm{D}_{11 / 2}-y^{2} \mathrm{D}_{21 / 2}^{\circ} \\
b^{4} \mathrm{D}_{21 / 2}-z^{4} \mathrm{P}_{21 / 2}^{\circ}\end{array}$ & $\begin{array}{ll}(0.00) & 1.23 \dagger \\
(0.79) & 0.89,1.37,1.85 \dagger\end{array}$ \\
\hline
\end{tabular}


TABLE 1. Wavelengths of $\mathrm{Cr}$ II in air-Continued

\begin{tabular}{|c|c|c|c|c|}
\hline Wavelength & Intensity & Wave No. & Term combination & Zeeman effect \\
\hline 4112.58 & 1 & 24308. 80 & $b{ }^{4} \mathrm{D}_{11 / 2}-z{ }^{4} \mathrm{P}_{21 / 2}^{\circ}$ & \\
\hline 4111.00 & 18 & 24318. 14 & $\begin{array}{l}b^{4} \mathrm{P}_{11 / 2}-z^{4} \mathrm{D}_{21 / 2}^{\circ} \\
b^{4} \mathrm{D}_{31 / 2}-z^{4} \mathrm{P}_{21 / 2}^{0}\end{array}$ & $(\mathbf{0 . 1 8}, 0.44) \mathbf{0 . 8 9}, 1.16 \dagger$ \\
\hline $\begin{array}{l}\text { 4098. } 44 \\
\text { 4089. } 48 \\
\text { 4088. } 88\end{array}$ & $\begin{array}{l}8 \\
2 \\
1\end{array}$ & $\begin{array}{l}\text { 24392. } 67 \\
24446.05 \\
24449.70\end{array}$ & $\begin{array}{l}b^{2} \mathrm{D}_{11 / 2}-y^{4} \mathrm{~F}_{11 / 2}^{21 / 2} \\
b^{2} \mathrm{D}_{21 / 2}-y^{4} \mathrm{G}_{21 / 2}^{\circ} \\
b^{4} \mathrm{D}_{11 / 2}-z^{6} \mathrm{D}_{01 / 2}^{0}\end{array}$ & $(0.53) 0.53,0.81 \dagger$ \\
\hline $\begin{array}{l}4087.60 \\
4086.14 \\
4082.30 \\
4081.21 \\
4077.50\end{array}$ & $\begin{array}{r}2 \\
8 \\
10 \\
1 \\
4\end{array}$ & $\begin{array}{l}24457.30 \\
24466.09 \\
24489.11 \\
24495.65 \\
24517.93\end{array}$ & $\begin{array}{c}b{ }^{4} \mathrm{D}_{01 / 2}-z^{6} \mathrm{D}_{01 / 2}^{\circ} \\
b^{4} \mathrm{P}_{01 / 2}-z^{4} \mathrm{D}_{01 / 2}^{0} \\
b^{2} \mathrm{D}_{21 / 2}-y^{4} \mathrm{~F}_{21 / 2}^{\circ} \\
b^{2} \mathrm{D}_{21 / 2}-y{ }^{4} \mathrm{~F}_{31 / 2}^{\circ} \\
b^{4} \mathrm{D}_{21 / 2}-z{ }^{6} \mathrm{D}_{11 / 2}^{\circ}\end{array}$ & $\begin{array}{l}(1.34) 1.35 \dagger \\
(0.38) / 1.10 \dagger\end{array}$ \\
\hline $\begin{array}{l}4076.87 \\
4072.56 \\
4070.88 \\
4056.07 \\
4054.10\end{array}$ & $\begin{array}{r}3 \\
4 \\
10 \\
4 \\
8\end{array}$ & $\begin{array}{l}24521.72 \\
24547.67 \\
24557.80 \\
24647.47 \\
24659.45\end{array}$ & $\begin{array}{c}b^{4} \mathrm{D}_{11 / 2}-z^{6} \mathrm{D}_{11 / 2}^{\circ} \\
b^{4} \mathrm{P}_{01 / 2}-z^{4} \mathrm{D}_{11 / 2}^{\circ} \\
d^{2} \mathrm{G}_{41 / 2}-w^{2} \mathrm{~F}_{31 / 2}^{\circ} \\
c^{2} \mathrm{D}_{11 / 2}-x^{4} \mathrm{G}_{21 / 2}^{\circ} \\
b^{4} \mathrm{D}_{21 / 2}-z^{6} \mathrm{D}_{21 / 2}^{\circ}\end{array}$ & $\begin{array}{l}(0.74) 0.46 \\
(0.00) 1.08 \dagger \\
(0.00 w) 0.37 A \dagger\end{array}$ \\
\hline $\begin{array}{l}4053.43 \\
4051.96 \\
4049.14 \\
4038.02 \\
4022.37\end{array}$ & $\begin{array}{r}1 \\
12 \\
18 \\
25 \\
3\end{array}$ & $\begin{array}{l}24663.52 \\
24672.47 \\
24689.65 \\
24757.64 \\
24853.97\end{array}$ & $\begin{array}{l}b^{4} \mathrm{D}_{11 / 2}-z^{6} \mathrm{D}_{21 / 2}^{\circ} \\
b^{4} \mathrm{D}_{31 / 2}-z^{6} \mathrm{D}_{21 / 2}^{2} \\
d^{2} \mathrm{G}_{31 / 2}-w^{2} \mathrm{~F}_{21 / 2}^{\circ} \\
d^{2} \mathrm{G}_{41 / 2}-w^{2} \mathrm{H}_{51 / 2}^{\circ} \\
c^{2} \mathrm{D}_{21 / 2}-y^{2} \mathrm{~F}_{21 / 2}^{\circ}\end{array}$ & $\begin{array}{l}(0.00 w) 1.16 \dagger \\
(0.00) 0.97 \dagger \\
(0.00) 1.06 \dagger\end{array}$ \\
\hline $\begin{array}{l}4017.96 \\
4012.50 \\
4007.55 \\
4003.32 \\
4002.48\end{array}$ & $\begin{array}{r}3 \\
30 \\
2 \\
25 \\
5\end{array}$ & $\begin{array}{l}\text { 24881. } 24 \\
24915.10 \\
24945.88 \\
24972.23 \\
24977.47\end{array}$ & $\begin{array}{l}b^{2} \mathrm{D}_{11 / 2}-x^{4} \mathrm{D}_{21 / 2}^{\circ} \\
c^{2} \mathrm{D}_{11 / 2}-y^{2} \mathrm{~F}_{21 / 2}^{\circ} \\
d^{2} \mathrm{G}_{31 / 2}-w^{2} \mathrm{H}_{41 / 2}^{\circ} \\
b^{2} \mathrm{D}_{21 / 2}-x^{4} \mathrm{D}_{31 / 2}^{\circ}\end{array}$ & $\begin{array}{ll}(0.00) & 0.90 \dagger \\
& \\
(0.00) & 0.95 \dagger \\
(0.00) & 0.73 \dagger\end{array}$ \\
\hline $\begin{array}{l}3979.52 \\
3936.95 \\
3935.04 \\
3911.32 \\
3909.25\end{array}$ & $\begin{array}{r}20 \\
1 \\
1 \\
3 \\
1\end{array}$ & $\begin{array}{l}25121.58 \\
25393.21 \\
25405.54 \\
25559.60 \\
25573.14\end{array}$ & $\begin{array}{l}c^{2} \mathrm{D}_{21 / 2}-y^{2} \mathrm{~F}_{31 / 2}^{\circ} \\
c^{2} \mathrm{G}_{41 / 2}-z^{4} \mathrm{I}_{41 / 2}^{\circ} \\
c^{4} \mathrm{D}_{01 / 2}-y^{4} \mathrm{D}_{01 / 2}^{\circ} \\
c^{2} \mathrm{G}_{41 / 2}-z^{4} \mathrm{G}_{41 / 2}^{\circ} \\
c^{2} \mathrm{G}_{31 / 2}-z^{4} \mathrm{G}_{31 / 2}^{\circ}\end{array}$ & $\begin{array}{l}(0.00) 1.07 \dagger \\
(0.00) 1.11 \dagger\end{array}$ \\
\hline $\begin{array}{l}\text { 3905. } 64 \\
3895.14 \\
3892.15 \\
3866.54 \\
3866.01\end{array}$ & $\begin{array}{r}25 \\
2 \\
4 \\
7 \\
5\end{array}$ & $\begin{array}{l}25596.77 \\
25665.78 \\
25685.49 \\
25855.61 \\
25859.16\end{array}$ & $\begin{array}{l}b^{2} \mathrm{D}_{11 / 2}-z^{2} \mathrm{~F}_{21 / 2}^{\circ} \\
c^{4} \mathrm{D}_{01 / 2}-y^{4} \mathrm{D}_{11 / 2}^{112} \\
b^{2} \mathrm{D}_{21 / 2}-z^{2} \mathrm{~F}_{21 / 2}^{\circ} \\
c^{2} \mathrm{G}_{41 / 2}-z^{2} \mathrm{G}_{41 / 2}^{\circ} \\
c^{2} \mathrm{G}_{31 / 2}-z^{2} \mathrm{G}_{31 / 2}^{\circ}\end{array}$ & $\begin{array}{l}(0.00) 0.94 A \dagger \\
(0.00) 1.18 \dagger\end{array}$ \\
\hline $\begin{array}{l}\text { 3865. } 60 \\
\text { 3832. } 40 \\
3813.99 \\
3810.74 \\
3801.21\end{array}$ & $\begin{array}{r}25 \\
1 \\
12 \\
3 \\
10\end{array}$ & $\begin{array}{l}25861.90 \\
26085.94 \\
26211.85 \\
26234.20 \\
26299.97\end{array}$ & $\begin{array}{l}b^{2} \mathrm{D}_{21 / 2}-z^{2} \mathrm{~F}_{31 / 2}^{\circ} \\
c^{4} \mathrm{D}_{11 / 2}-y^{4} \mathrm{D}_{21 / 2}^{\circ} \\
d^{2} \mathrm{~F}_{31 / 2}-w^{2} \mathrm{~F}_{31 / 2}^{\circ} \\
a^{2} \mathrm{~S}_{01 / 2}-z^{2} \mathrm{D}_{11 / 2}^{\circ} \\
d^{2} \mathbf{F}_{21 / 2}-w^{2} \mathbf{F}_{21 / 2}^{\circ}\end{array}$ & $\begin{array}{l}(0.00) 1.09 \dagger \\
(0.00) 1.17 \dagger \\
(0.00) 0.86 \dagger\end{array}$ \\
\hline $\begin{array}{l}3783.74 \\
3778.70 \\
3769.32 \\
3766.65 \\
3765.61\end{array}$ & $\begin{array}{l}6 \\
6 \\
1 \\
2 \\
8\end{array}$ & $\begin{array}{l}\text { 26421. } 40 \\
26456.64 \\
26522.48 \\
26541.28 \\
26548.61\end{array}$ & $\begin{array}{l}a^{2} \mathrm{~S}_{01 / 2}-z^{2} \mathrm{P}_{01 / 2}^{\circ} \\
b^{4} \mathrm{D}_{11 / 2}-z^{4} \mathrm{~F}_{11 / 2}^{\circ} \\
b^{4} \mathrm{D}_{01 / 2}-z^{4} \mathrm{~F}_{11 / 2}^{112}\end{array}$ & $\begin{array}{l}(0.64) 1.39 \dagger \\
(0.17) 0.24, \mathbf{0 . 5 8} \dagger\end{array}$ \\
\hline 3761.90 & 8 & 26574. 79 & $\begin{array}{l}a^{4} \mathrm{P}_{01 / 2}-z^{6} \mathrm{P}_{11 / 2}{ }^{\circ} \\
a^{4} \mathrm{P}_{11 / 2}-z^{6} \mathrm{P}_{11 / 2}^{\circ}\end{array}$ & $\mathrm{P}-\mathrm{B}$ \\
\hline $\begin{array}{l}3761.68 \\
3756.55 \\
3755.13 \\
3754.59\end{array}$ & $\begin{array}{r}7 \\
3 \\
2 \\
20\end{array}$ & $\begin{array}{l}\text { 26576. } 34 \\
26612.63 \\
26622.70 \\
26626.53\end{array}$ & $\begin{array}{l}a^{4} \mathrm{P}_{21 / 2}^{11 / 2}-z^{6} \mathrm{P}_{11 / 2}^{11 / 2} \\
c^{2} \mathrm{~F}_{21 / 2}-y^{4} \mathrm{P}_{11 / 2}^{1} \\
b^{4} \mathrm{D}_{21 / 2}-z^{4} \mathrm{~F}_{21 / 2}^{0} \\
b^{4} \mathrm{D}_{11 / 2}-z^{4} \mathrm{~F}_{21 / 2}^{0}\end{array}$ & $\begin{array}{l}\mathrm{P}-\mathrm{B} \\
(0.00) 0.83 \dagger\end{array}$ \\
\hline $\begin{array}{l}3750.99 \\
3750.61\end{array}$ & $\frac{1}{5}$ & $\begin{array}{l}\text { 26652. } 02 \\
26654.71\end{array}$ & $b^{2} \mathrm{D}_{11 / 2}-y^{2} \mathrm{D}^{1}{ }^{1} / 2$ & . \\
\hline 3750.56 & 7 & 26655. 14 & $\left\{\begin{array}{c}a^{2} \mathrm{~S}_{01 / 2}-z^{2} \mathrm{P}_{11 / 2}^{11 / 2} \\
c^{4} \mathrm{D}_{31 / 2}-y^{4} \mathrm{D}_{31 / 2}^{\circ}\end{array}\right.$ & $(0.39) 0.82,1.61$ \\
\hline $\begin{array}{l}3748.68 \\
3735.38\end{array}$ & $3 \stackrel{7}{5}$ & $\begin{array}{l}26668.50 \\
26741.98\end{array}$ & $\begin{array}{l}a^{4} \mathrm{P}_{21 / 2}-z^{6} \mathrm{P}_{21 / 2}^{\circ} \\
c^{4} \mathrm{D}_{21 / 2}-z^{4} \mathrm{~F}_{31 / 2}^{\circ}\end{array}$ & $(0.00,0.15,0.30) 0.90,1.05,1.21$ \\
\hline $\begin{array}{l}3737.55 \\
3736.56 \\
3735.89 \\
3727.36 \\
3723.40\end{array}$ & $\begin{array}{r}10 \\
1 \\
4 \\
25 \\
15\end{array}$ & $\begin{array}{l}26747.92 \\
26755.00 \\
26759.80 \\
26821.04 \\
26849.56\end{array}$ & $\begin{array}{l}b^{2} \mathrm{G}_{31 / 2}-z^{4} \mathrm{G}_{31 / 2}^{\circ} \\
b^{4} \mathrm{D}_{31 / 2}-z^{4} \mathrm{~F}_{31 / 2}^{0} \\
d^{2} \mathrm{D}_{21 / 2}-x^{2} \mathrm{~F}_{31 / 2}^{\circ} \\
b^{2} \mathrm{G}_{41 / 2}-z^{4} \mathrm{G}_{41 / 2}^{\circ} \\
c^{2} \mathrm{~F}_{31 / 2}-y^{4} \mathrm{P}_{21 / 2}^{0}\end{array}$ & $\begin{array}{l}(0.22) 0.92 \dagger \\
(0.00) 0.96 \dagger \\
(0.00) 1.12 \dagger \\
(\mathbf{0 . 2 5}, 0.75,1.19) \mathbf{0 . 2 4}, 0.81 \dagger\end{array}$ \\
\hline
\end{tabular}


TABLE 1. Wavelengths of $\mathrm{Cr}$ II in air-Continued

\begin{tabular}{|c|c|c|c|c|}
\hline Wavelength & Intensity & Wave No. & Term combination & Zeeman effect \\
\hline $\begin{array}{l}3715.44 \\
3715.18 \\
3712.95 \\
3712.89 \\
3711.29\end{array}$ & $\begin{array}{r}20 \\
25 \\
45 \\
10 \\
7\end{array}$ & $\begin{array}{l}\text { 26907. } 09 \\
26908.97 \\
26925.15 \\
26925.56 \\
26937.17\end{array}$ & $\begin{array}{l}c^{2} \mathrm{~F}_{21 / 2}-z^{2} \mathrm{D}_{11 / 2}^{\circ} \\
b^{4} \mathrm{D}_{31 / 2}-z^{4} \mathrm{~F}_{41 / 2}^{01} \\
a^{4} \mathrm{P}_{11 / 2}-z^{4} \mathrm{P}_{01 / 2}^{\circ} \\
a^{4} \mathrm{P}_{01 / 2}-z^{4} \mathrm{P}_{01 / 2}^{0} \\
e^{2} \mathrm{D}_{21 / 2}-v^{2} \mathrm{D}_{21 / 2}^{\circ}\end{array}$ & $\begin{array}{l}(0.00) 0.82 \dagger \\
(0.00) 1.10 A \dagger \\
\mathrm{P}-\mathrm{B} \\
\mathrm{P}-\mathrm{B} \\
(0.00) 1.05 \dagger\end{array}$ \\
\hline $\begin{array}{l}3707.57 \\
3707.13 \\
3704.89 \\
3701.89\end{array}$ & $\begin{array}{l}1 \\
3 \\
5 \\
4\end{array}$ & $\begin{array}{l}\text { 26964. } 20 \\
\text { 26967. } 40 \\
\text { 26983. } 70 \\
\text { 27005. } 57\end{array}$ & $\begin{array}{l}a^{2} \mathrm{~S}_{01 / 2}-y^{4} \mathrm{~F}_{11 / 2}^{\circ} \\
b^{2} \mathrm{D}_{11 / 2}-y^{2} \mathrm{D}_{21 / 2}^{\circ} \\
e^{2} \mathrm{D}_{11 / 2}-v^{2} \mathrm{D}_{11 / 2}^{\circ} \\
b^{2} \mathrm{D}_{21 / 2}-y^{2} \mathrm{G}_{31 / 2}^{\circ}\end{array}$ & \\
\hline $\begin{array}{l}\text { 3698. } 01 \\
\text { 3696. } 79 \\
3695.53 \\
3694.98 \\
3693.98\end{array}$ & $\begin{array}{r}35 \\
10 \\
2 \\
4 \\
3\end{array}$ & $\begin{array}{l}27033.91 \\
27042.83 \\
27052.05 \\
27056.07 \\
27063.40\end{array}$ & $\begin{array}{l}b^{2} \mathrm{G}_{31 / 2}-z^{2} \mathrm{G}_{31 / 2}^{\circ} \\
b^{2} \mathrm{D}_{21 / 2}-y^{2} \mathrm{D}_{21 / 2}^{\circ} \\
d^{2} \mathrm{D}_{11 / 2}-x^{2} \mathrm{~F}_{21 / 2}^{\circ}\end{array}$ & $\begin{array}{l}(0.00) 0.91 \dagger \\
(\mathbf{0 . 2 4}, 0.74) 0.27 \dagger\end{array}$ \\
\hline $\begin{array}{l}\text { 3686. } 68 \\
3684.25 \\
3677.90 \\
3677.84 \\
3677.67\end{array}$ & $\begin{array}{l}20 \\
25 \\
30 \\
50 \\
40\end{array}$ & $\begin{array}{l}27116.98 \\
27134.87 \\
27181.72 \\
27182.16 \\
27183.39\end{array}$ & $\begin{array}{l}b^{2} \mathrm{G}_{41 / 2}-z^{2} \mathrm{G}_{41 / 2}^{\circ} \\
c^{2} \mathrm{~F}_{31 / 2}-z^{2} \mathrm{D}_{21 / 2}^{\circ} \\
a^{4} \mathrm{P}_{11 / 2}-z^{4} \mathrm{P}_{11 / 2}^{\circ} \\
a^{4} \mathrm{P}_{01 / 2}-z^{4} \mathbf{P}_{11 / 2}^{\circ} \\
a^{4} \mathrm{P}_{21 / 2}-z^{4} \mathrm{P}_{11 / 2}^{\circ}\end{array}$ & $\begin{array}{l}(0.00) 1.13 \dagger \\
\text { P-B } \\
\text { P-B } \\
\text { P-B }\end{array}$ \\
\hline $\begin{array}{l}3674.94 \\
3664.95 \\
3661.44\end{array}$ & $\begin{array}{r}5 \\
30 \\
3\end{array}$ & $\begin{array}{l}27203.61 \\
27277.76 \\
27303.91\end{array}$ & $\begin{array}{c}a^{4} \mathrm{D}_{31 / 2}-z^{6} \mathrm{~F}_{31 / 2}^{\circ} \\
b^{2} \mathrm{I}_{51 / 2}-z^{2} \mathrm{I}_{51 / 2}^{\circ} \\
b^{2} \mathrm{I}_{61 / 2}-z^{2} \mathrm{I}_{51 / 2}^{\circ}\end{array}$ & $(0.00) 0.93 \uparrow$ \\
\hline 3658.19 & 20 & 27328. 16 & $\begin{array}{l}c^{2} \mathrm{~F}_{21 / 2}-z^{2} \mathrm{P}_{11 / 2} \\
c^{2} \mathrm{G}_{21}-z^{3} \mathrm{D}^{\circ}\end{array}$ & $\left(\mathbf{0 . 1 5}, \mathbf{r}_{0} 0.38,0.61\right) \mathbf{0 . 3 5}, 0.61,0.88 \dagger$ \\
\hline 3657. 93 & 1 & 27330. 11 & $b^{2} \mathrm{D}_{11 / 2}-x{ }^{4} \mathrm{G}_{21 / 2}^{272}$ & \\
\hline 3651.68 & 12 & 27376. 88 & $\left\{\begin{array}{c}a{ }^{4} \mathrm{D}_{01 / 2}-z^{6} \mathrm{~F}_{1 / 2}^{\circ} \\
b^{2} \mathrm{P}_{11 / 2}-v^{2} \mathrm{D}_{21 / 2}^{\circ}\end{array}\right.$ & $(00.0 d, 0.53) \mathbf{1 . 0 8}, 1.60 \dagger$ \\
\hline $\begin{array}{l}\text { 3650. } 37 \\
3649.69 \\
3647.40 \\
3644.70\end{array}$ & $\begin{array}{l}40 \\
7 w \\
8 \\
10\end{array}$ & $\begin{array}{l}\text { 27386. } 71 \\
27391.81 \\
27409.01 \\
27429.31\end{array}$ & $\begin{array}{l}b^{2} \mathrm{I}_{61 / 2}-z^{2} \mathrm{I}_{61 / 2}^{0} 0 \\
a^{4} \mathrm{D}_{11 / 2}-z^{6} \mathrm{~F}_{21 / 2}^{0} \\
a^{4} \mathrm{D}_{21 / 2}-z^{6} \mathrm{~F}_{31 / 2}^{\circ}\end{array}$ & $\begin{array}{l}(0.00) 1.10 \dagger \\
(0.00) 1.44 \dagger \\
(0.00) 1.43 \dagger\end{array}$ \\
\hline $\begin{array}{l}\text { 3643. } 22 \\
3634.04 \\
3631.70 \\
3631.49 \\
3622.44\end{array}$ & $\begin{array}{r}10 \\
10 \\
40 \\
50 \\
1\end{array}$ & $\begin{array}{l}27440.38 \\
27509.77 \\
27527.52 \\
27529.12 \\
27597.86\end{array}$ & $\begin{array}{l}a{ }^{4} \mathrm{D}_{31 / 2}-z^{6} \mathrm{~F}_{41 / 2}^{\circ} \\
c^{2} \mathrm{G}_{41 / 2}-y^{4} \mathrm{G}_{31 / 2}^{\circ} \\
a^{4} \mathrm{P}_{11 / 2}-z^{4} \mathrm{P}_{21 / 2}^{\circ} \\
a^{4} \mathrm{P}_{21 / 2}-z^{4} \mathrm{P}_{21 / 2}^{\circ} \\
b^{2} \mathrm{D}_{11 / 2}^{\circ}-y^{2} \mathrm{~F}_{21 / 2}^{\circ}\end{array}$ & $\begin{array}{l}(0.00) 1.45 \dagger \\
(0.00) 1.05 \dagger \\
\mathrm{P}-\mathrm{B} \\
\mathrm{P}-\mathrm{B}\end{array}$ \\
\hline $\begin{array}{l}3617.33 \\
3614.25 \\
3613.33 \\
3613.20 \\
3608.66\end{array}$ & $\begin{array}{r}7 \\
2 \\
15 \\
20 \\
3\end{array}$ & $\begin{array}{l}27636.85 \\
27660.40 \\
27667.44 \\
27668.44 \\
27703.24\end{array}$ & $\begin{array}{l}c^{2} \mathrm{~F}_{21 / 2}-y{ }^{4} \mathrm{~F}_{11 / 2}^{\circ} \\
c^{2} \mathrm{G}_{31 / 2}-y^{4} \mathrm{G}_{21 / 2} \\
a^{4} \mathrm{P}_{11 / 2}-z^{6} \mathrm{D}_{01 / 2}^{\circ} \\
a^{4} \mathrm{P}_{01 / 2}-z^{6} \mathrm{D}_{01 / 2}^{\circ} \\
c^{2} \mathrm{G}_{31 / 2}-y{ }^{4} \mathrm{~F}_{21 / 2}^{\circ}\end{array}$ & $\begin{array}{l}(\mathbf{0 . 1 7}, 0.54) 0.71,1.04,1.36 \dagger \\
\text { P-B } \\
\text { P-B }\end{array}$ \\
\hline $\begin{array}{l}3603.86 \\
3603.78 \\
3603.62 \\
3588.30 \\
3585.53\end{array}$ & $\begin{array}{r}20 \\
40 \\
20 \\
2 \\
40\end{array}$ & $\begin{array}{l}27740.14 \\
27740.75 \\
27741.99 \\
27860.43 \\
27881.95\end{array}$ & $\begin{array}{l}a^{4} \mathrm{P}_{11 / 2}-z^{6} \mathrm{D}_{11 / 2}^{\circ} \\
a^{4} \mathrm{P}_{01 / 2}-z^{6} \mathrm{D}_{11 / 2}^{\circ} \\
a^{4} \mathrm{P}_{21 / 2}-z^{6} \mathrm{D}_{11 / 2}^{\circ} \\
c^{4} \mathrm{D}_{01 / 2}-y^{4} \mathrm{P}_{01 / 2}^{\circ} \\
a^{4} \mathrm{P}_{11 / 2}-z^{6} \mathrm{D}_{21 / 2}^{\circ}\end{array}$ & $\begin{array}{l}\mathrm{P}-\mathrm{B} \\
\mathrm{P}-\mathrm{B} \\
\mathrm{P}-\mathrm{B} \\
\mathrm{P}-\mathrm{B}\end{array}$ \\
\hline $\begin{array}{l}\text { 3585. } 30 \\
3583.96 \\
3571.37 \\
3566.37 \\
3565.32\end{array}$ & $\begin{array}{r}60 \\
3 \\
3 \\
1 \\
5\end{array}$ & $\begin{array}{l}27883.74 \\
27894.16 \\
27992.50 \\
28031.74 \\
28039.99\end{array}$ & $\begin{array}{l}a^{4} \mathrm{P}_{21 / 2}-z^{6} \mathrm{D}_{21 / 2}^{\circ} \\
c^{4} \mathrm{D}_{11 / 2}-y^{4} \mathrm{P}_{011 / 2}^{\circ} \\
c^{4} \mathrm{D}_{11 / 2}-y^{4} \mathrm{P}_{11 / 2}^{\circ} \\
b^{2} \mathrm{~F}_{21 / 2}-z^{4} \mathrm{H}_{31 / 2}^{0} \\
c^{4} \mathrm{D}_{21 / 2}-y^{4} \mathrm{P}_{11 / 2}^{\circ}\end{array}$ & $(\mathbf{0 . 1 7}, 0.47) \mathbf{0 . 6 9}, 0.97 \dagger$ \\
\hline $\begin{array}{l}3563.91 \\
3552.42 \\
3547.10 \\
3540.23 \\
3538.98\end{array}$ & $\begin{array}{l}5 \\
2 \\
3 \\
2 \\
4\end{array}$ & $\begin{array}{l}28051.09 \\
28141.82 \\
28184.02 \\
28238.71 \\
28248.68\end{array}$ & $\begin{array}{c}c^{2} \mathrm{G}_{41 / 2}-x{ }^{4} \mathrm{D}_{31 / 2}^{\circ} \\
b^{2} \mathrm{H}_{51 / 2}-z^{4} \mathrm{H}_{51 / 2}^{\circ} \\
c^{2} \mathrm{G}_{31 / 2}-x^{4} \mathrm{D}_{21 / 2}^{\circ} \\
b^{2} \mathrm{H}_{41 / 2}-z^{4} \mathrm{H}_{51 / 2}^{\circ} \\
b^{2} \mathbf{I}_{51 / 2}-z^{2} \mathrm{H}_{41 / 2}^{01 / 2}\end{array}$ & \\
\hline $\begin{array}{l}3538.47 \\
3534.13 \\
3529.73 \\
3528.23 \\
3522.13\end{array}$ & $\begin{array}{l}1 \\
2 \\
2 \\
1 \\
7\end{array}$ & $\begin{array}{l}\text { 28252. } 76 \\
28287.45 \\
28322.71 \\
28334.75 \\
28383.83\end{array}$ & $\begin{array}{l}c^{4} \mathrm{D}_{01 / 2}-z^{2} \mathrm{D}_{11 / 2}^{\circ} \\
c^{4} \mathrm{D}_{11 / 2}-z^{2} \mathrm{D}_{11 / 2}^{1} \\
b^{2} \mathrm{H}_{51 / 2}-z^{4} \mathrm{H}_{61 / 2}^{\circ} \\
c^{4} \mathrm{D}_{21 / 2}-z^{2} \mathrm{D}_{11 / 2}^{\circ} \\
c^{2} \mathrm{D}_{21 / 2}-x^{2} \mathrm{~F}_{31 / 2}^{\circ}\end{array}$ & $(0.00) 1.08 \dagger$ \\
\hline
\end{tabular}


TABLE 1. Wavelengths of $\mathrm{Cr}$ II in air-Continued

\begin{tabular}{|c|c|c|c|c|}
\hline Wavelength & Intensity & Wave No. & Term combination & Zeeman effect \\
\hline $\begin{array}{l}\text { 3518. } 62 \\
3513.03 \\
3511.83 \\
3506.61 \\
3503.35\end{array}$ & $\begin{array}{r}3 \\
10 \\
35 \\
1 \\
3\end{array}$ & $\begin{array}{l}28412.14 \\
28457.35 \\
28467.07 \\
28509.45 \\
28535.97\end{array}$ & $\begin{array}{c}c^{4} \mathrm{D}_{21 / 2}-y{ }^{4} \mathrm{P}_{21 / 2}^{\circ} \\
c^{4} \mathrm{D}_{31 / 2}-y^{4} \mathrm{P}_{21 / 2}^{\circ} \\
a^{4} \mathrm{D}_{31 / 2}-z^{6} \mathrm{P}_{21 / 2}^{o} \\
b^{2} \mathrm{I}_{51 / 2}-z^{2} \mathrm{H}_{51 / 2}^{\circ} \\
b^{2} \mathrm{I}_{61 / 2}-z^{2} \mathrm{H}_{51 / 2}^{\circ}\end{array}$ & $\begin{array}{l}(0.00) 1.30 \dagger \\
(\mathbf{0 . 2 3}, 0.69,1.13) \mathbf{0 . 3 0}, 0.74,1.20,1.64,2.11\end{array}$ \\
\hline $\begin{array}{l}\text { 3498. } 30 \\
3495.54 \\
3495.36 \\
3494.50 \\
3489.44\end{array}$ & $\begin{array}{r}1 \\
20 \\
25 \\
4 \\
2\end{array}$ & $\begin{array}{l}\text { 28577. } 17 \\
\text { 28599. } 73 \\
\text { 28601. } 20 \\
\text { 28608. } 24 \\
\text { 28649. } 74\end{array}$ & $\left\{\begin{array}{c}c^{2} \mathrm{~F}_{31 / 2}-z^{2} \mathrm{H}_{41 / 2}^{\circ} \\
a^{4} \mathrm{D}_{21 / 2}-z^{6} \mathrm{P}_{11 / 2}^{\circ} \\
a^{4} \mathrm{D}_{31 / 2}-z^{6} \mathrm{P}_{31 / 2}^{\circ} \\
c^{4} \mathrm{D}_{11 / 2}-z^{2} \mathrm{D}_{21 / 2}^{\circ} \\
c^{2} \mathrm{D}_{11 / 2}-w^{4} \mathrm{~F}_{21 / 2}^{\circ}\end{array}\right.$ & $(\mathbf{0 . 5 1}, 1.51)-\mathbf{0 . 1 4}, 0.86,1.86,2.87$ \\
\hline $\begin{array}{l}\text { 3489. } 07 \\
3484.14\end{array}$ & $\begin{array}{r}2 \\
20\end{array}$ & $\begin{array}{l}\text { 28652. } 76 \\
\text { 28693. } 30\end{array}$ & $\begin{array}{l}c^{2} \mathrm{G}_{41 / 2}-z^{2} \mathrm{H}_{41 / 2}^{\circ} \\
a^{4} \mathrm{D}_{21 / 2}-z^{6} \mathrm{P}_{21 / 2}^{0}\end{array}$ & $(0.27,0.76,1.28) 0.61,1.13,1.63,2.14,2.65$ \\
\hline 3482.58 & 12 & 28706. 16 & $\begin{array}{l}c^{2} \mathrm{D}_{21 / 2}-x^{2} \mathrm{~F}_{21 / 2}^{\circ} \\
c^{2} \mathrm{~F}_{31 / 2}-z^{2} \mathrm{~F}_{21 / 2}^{2}\end{array}$ & $(0.00 w) 1.40 B \dagger$ \\
\hline 3478.15 & 3 & 28742. 72 & $c^{4} \mathrm{D}_{31 / 2}^{31 / 2}-z^{2} \mathrm{D}_{21 / 2}^{2}$ & \\
\hline 3475. 12 & 20 & 28767. 78 & $\left\{\begin{array}{l}c^{2} \mathrm{D}_{11 / 2}-x^{2} \mathrm{~F}_{21 / 2}^{\circ} \\
a^{4} \mathrm{D}_{11 / 2}-z^{6} \mathrm{P}_{11 / 2}^{\circ}\end{array}\right.$ & $(0.58,1.78) 0.61,1.79,2.98 \dagger$ \\
\hline 3472.06 & 25 & 28793. 13 & $c^{2} \mathrm{G}_{31 / 2}-z^{2} \mathrm{H}_{41 / 2}^{\circ}$ & $\left(0.00 w_{1} D\right) 0.64 A$ \\
\hline 3466.25 & 2 & 28841. 39 & $\begin{array}{l}c^{2} \mathrm{~F}_{21 / 2}-z^{2} \mathrm{~F}_{21 / 2}^{\circ} \\
b^{2} \mathrm{~F}_{31 / 2}-y^{4} \mathrm{D}_{21 / 2}^{\circ}\end{array}$ & \\
\hline $\begin{array}{l}3464.01 \\
3462.71 \\
3461.28\end{array}$ & $\begin{array}{l}4 \\
6 \\
3\end{array}$ & $\begin{array}{l}28860.04 \\
28870.87 \\
28882.80\end{array}$ & $\begin{array}{c}a^{4} \mathrm{D}_{11 / 2}-z^{6} \mathrm{P}_{21 / 2} \\
a^{4} \mathrm{D}_{01 / 2}-z^{6} \mathrm{P}_{11 / 2}^{11 / 2} \\
c^{2} \mathrm{~F}_{31 / 2}-z^{2} \mathrm{~F}_{31 / 2}^{\circ}\end{array}$ & $(1.18) 1.15,3.59 \dagger$ \\
\hline $\begin{array}{l}3460.03 \\
3459.28 \\
3457.61 \\
3454.97 \\
3450.84\end{array}$ & $\begin{array}{r}1 \\
25 \\
30 \\
35 \\
3\end{array}$ & $\begin{array}{l}\text { 28893. } 24 \\
28899.50 \\
28913.46 \\
28935.55 \\
28970.18\end{array}$ & $\begin{array}{c}a^{2} \mathrm{H}_{51 / 2}-z^{4} \mathrm{H}_{41 / 2}^{\circ} \\
c^{2} \mathrm{G}_{31 / 2}-z^{2} \mathrm{~F}_{21 / 2}^{\circ} \\
c^{2} \mathrm{G}_{41 / 2}-z^{2} \mathrm{H}_{51 / 2}^{\circ} \\
c^{2} \mathrm{G}_{41 / 2}-z^{2} \mathrm{~F}_{31 / 2}^{\circ} \\
a^{2} \mathrm{H}_{41 / 2}-z^{4} \mathrm{H}_{31 / 2}^{\circ}\end{array}$ & $\begin{array}{l}\left(0.00 w_{1} D\right) 1.30 B \\
(0.00 w) 1.05 w \\
(0.00) 1.04 \dagger\end{array}$ \\
\hline $\begin{array}{l}3449.28 \\
3445.04 \\
3444.34 \\
3442.98 \\
3438.46\end{array}$ & $\begin{array}{l}1 \\
5 \\
4 \\
1 \\
1\end{array}$ & $\begin{array}{l}\text { 28983. } 29 \\
29018.95 \\
29024.85 \\
29036.32 \\
29074.48\end{array}$ & $\begin{array}{l}c^{4} \mathrm{D}_{01 / 2}-y{ }^{4} \mathrm{~F}_{11 / 2}^{\circ} \\
c^{2} \mathrm{G}_{41 / 2}-y^{4} \mathrm{H}_{31 / 2}^{\circ} \\
c^{4} \mathrm{D}_{11 / 2}-y{ }^{4} \mathrm{~F}_{21 / 2}^{\circ} \\
a^{2} \mathrm{H}_{51 / 2}-z^{4} \mathrm{H}_{51 / 2}^{\circ} \\
c^{4} \mathrm{D}_{31 / 2}-y^{4} \mathrm{G}_{21 / 2}^{\circ}\end{array}$ & \\
\hline $\begin{array}{l}3437.93 \\
3433.29 \\
3430.42 \\
3429.90 \\
3428.94\end{array}$ & $\begin{array}{r}2 \\
75 \\
3 \\
1 \\
7\end{array}$ & $\begin{array}{l}29078.95 \\
29118.26 \\
29142.62 \\
29147.04 \\
29155.20\end{array}$ & $\begin{array}{l}c^{4} \mathrm{D}_{21 / 2}-y{ }^{4} \mathrm{~F}_{31 / 2}^{\circ} \\
a^{4} \mathrm{D}_{11 / 2}-z^{4} \mathrm{P}_{01 / 2}^{\circ} \\
a^{2} \mathrm{P}_{01 / 2}-y^{4} \mathrm{D}_{01 / 2}^{\circ} \\
a^{2} \mathrm{G}_{41 / 2}-z^{4} \mathrm{I}_{51 / 2}^{\circ} \\
a^{2} \mathrm{G}_{31 / 2}-z^{4} \mathrm{G}_{31 / 2}^{\circ}\end{array}$ & $\begin{array}{l}(0.82) 0.37,2.03 \dagger \\
(0.31) 0.38 \dagger \\
(0.25) 0.97 \dagger\end{array}$ \\
\hline $\begin{array}{l}3426.14 \\
3422.73\end{array}$ & $\begin{array}{r}8 \\
125\end{array}$ & $\begin{array}{l}\text { 29179. } 10 \\
\text { 29208. } 28\end{array}$ & $\begin{array}{l}c^{4} \mathrm{D}_{31 / 2}-y{ }^{4} \mathrm{~F}_{41 / 2}^{\circ} \\
a^{4} \mathrm{D}_{21 / 2}-z^{4} \mathrm{P}_{11 / 2}^{\circ}\end{array}$ & $\begin{array}{l}(0.00 w) 1.05 \dagger \\
(\mathbf{0 . 2 1}, 0.65) 0.72,1.16,1.59,2.02\end{array}$ \\
\hline 3421.62 & 5 & 29217. 57 & $\begin{array}{l}a^{2} \mathrm{H}_{41 / 2}-z^{4} \mathrm{H}_{51 / 2}^{\circ} \\
a^{2} \mathrm{H}_{51 / 2}-z^{4} \mathrm{H}_{61 / 2}^{5}\end{array}$ & \\
\hline $\begin{array}{l}3421.19 \\
3415.44\end{array}$ & $\begin{array}{r}75 \\
2\end{array}$ & $\begin{array}{l}\text { 29221. } 24 \\
\text { 29270. } 44\end{array}$ & $\begin{array}{l}a^{4} \mathrm{D}_{01 / 2}-z^{4} \mathrm{P}_{01 / 2}^{61 / 2} \\
a^{2} \mathrm{G}_{41 / 2}-z^{2} \mathrm{G}_{31 / 2}^{\circ}\end{array}$ & $(1.40) 1.40$ \\
\hline $\begin{array}{l}3410.53 \\
3408.76 \\
3405.13\end{array}$ & $\begin{array}{r}3 \\
150 \\
2\end{array}$ & $\begin{array}{l}29312.58 \\
29327.80 \\
29359.06\end{array}$ & $\begin{array}{c}b^{2} \mathrm{G}_{41 / 2}-x^{4} \mathrm{D}_{31 / 2}^{\circ} \\
a^{4} \mathrm{D}_{31 / 2}-z^{4} \mathrm{P}_{21 / 2}^{\circ} \\
b^{2} \mathrm{G}_{31 / 2}-x^{4} \mathrm{D}_{21 / 2}^{\circ}\end{array}$ & $(\mathbf{0 . 1 0}, 0.32,0.53) \mathbf{0 . 9 0}, 1.11,1.32,1.53,1.74,1.94$ \\
\hline 3403.30 & 100 & 29374.85 & $\begin{array}{l}b{ }^{4} \mathrm{D}_{11 / 2}-z^{4} \mathrm{D}_{01 / 2}^{\circ} \\
a^{4} \mathrm{D}_{11 / 2}-z^{4} \mathrm{P}_{11 / 6}^{0}\end{array}$ & $(0.31, \mathbf{0 . 9 1}) 0.90,1.49,2.10$ \\
\hline 3402.43 & 25 & 29382. 36 & ${ }^{4} \mathrm{D}_{01 / 2}^{11 / 2}-z^{4} \mathrm{D}_{01 / 2}^{13 / 2}$ & $(0.00) 0.00 \dagger$ \\
\hline $\begin{array}{l}3400.08 \\
3399.54 \\
3395.62 \\
3394.31 \\
3393.85\end{array}$ & $\begin{array}{r}2 \\
18 \\
20 \\
35 \\
30\end{array}$ & $\begin{array}{l}\text { 29402. } 66 \\
\text { 29407. } 34 \\
\text { 29441. } 28 \\
\text { 29452. } 64 \\
29456.64\end{array}$ & $\begin{array}{l}a^{2} \mathrm{P}_{01 / 2}-y^{4} \mathrm{D}_{11 / 2}^{\circ} \\
a^{2} \mathrm{G}_{41 / 2}-z^{2} \mathrm{G}_{41 / 2}^{\circ} \\
a^{2} \mathrm{G}_{31 / 2}-z^{2} \mathrm{G}_{31 / 2}^{\circ} \\
b^{4} \mathrm{D}_{21 / 2}-z^{4} \mathrm{D}_{11 / 2}^{\circ} \\
b^{4} \mathrm{D}_{11 / 2}^{\circ}-z^{4} \mathrm{D}_{11 / 2}^{\circ}\end{array}$ & $\begin{array}{l}(0.13) 1.11 w \\
(0.00 w, d ?) 0.91 w \\
(0.17) 1.61 \dagger \\
(0.00) 1.18 \dagger\end{array}$ \\
\hline $\begin{array}{l}3393.00 \\
3391.41 \\
3389.17 \\
3387.95 \\
3387.72\end{array}$ & $\begin{array}{r}35 \\
35 \\
2 \\
3 \\
5\end{array}$ & $\begin{array}{l}\text { 29464. } 02 \\
29477.73 \\
29498.18 \\
29507.93 \\
29509.94\end{array}$ & $\begin{array}{l}b^{4} \mathrm{D}_{01 / 2}-z^{4} \mathrm{D}_{11 / 2}^{\circ} \\
a^{4} \mathrm{D}_{01 / 2}-z^{4} \mathrm{P}_{11 / 2}^{\circ} \\
c^{4} \mathrm{D}_{11 / 2}-x^{4} \mathrm{D}_{01 / 2}^{\circ} \\
c^{4} \mathrm{D}_{11 / 2}-x^{4} \mathrm{D}_{11 / 2}^{0} \\
b^{2} \mathrm{H}_{51 / 2}-z^{4} \mathrm{I}_{41 / 2}^{0}\end{array}$ & $\begin{array}{l}(0.59) 0.64,1.78 \dagger \\
(0.90) 0.92,2.73 \dagger\end{array}$ \\
\hline
\end{tabular}


TABLE 1. Wavelengths of $\mathrm{Cr}$ II in air-Continued

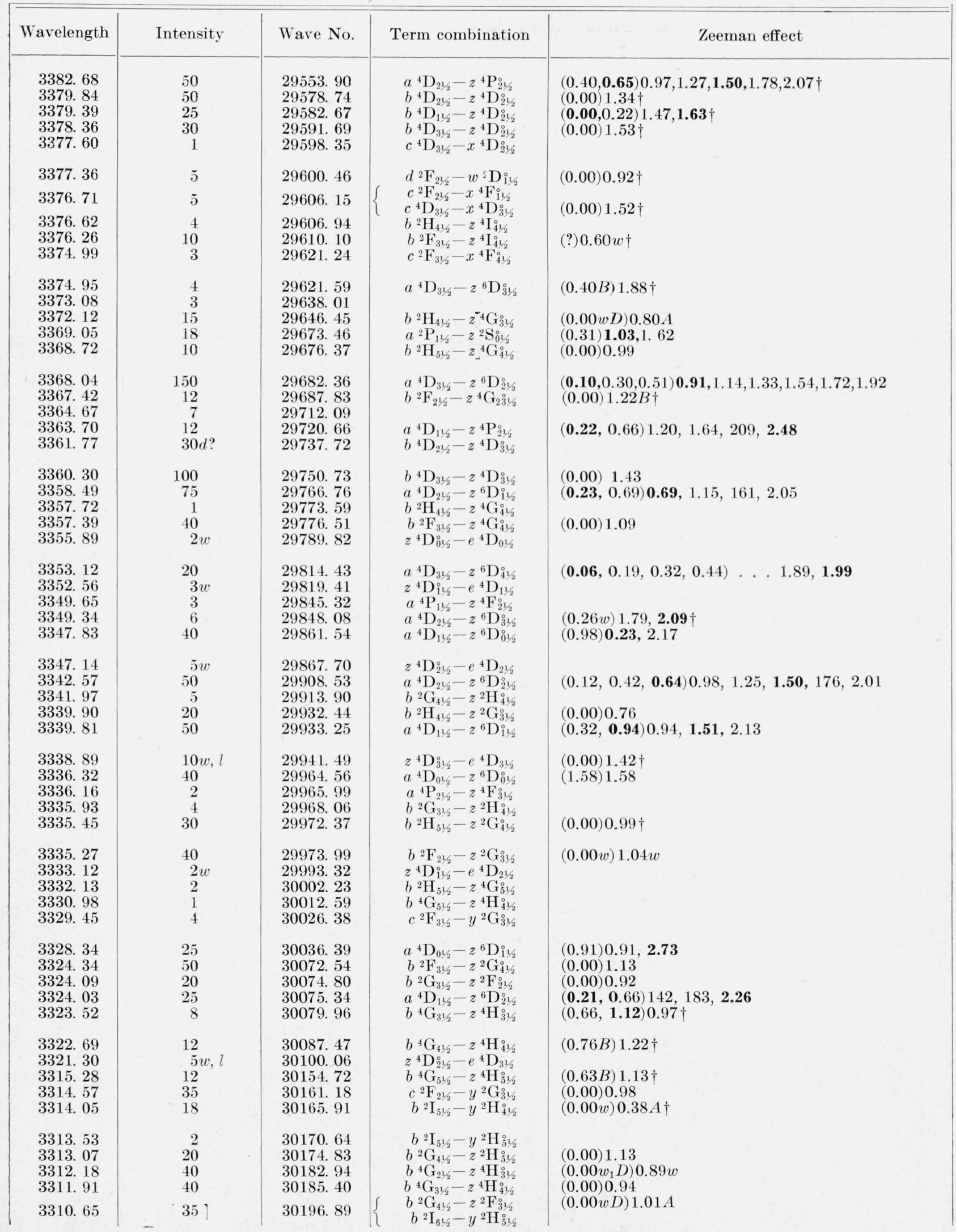


TABLE 1. Wavelengths of $\mathrm{Cr}$ II in air-Continued

\begin{tabular}{|c|c|c|c|c|}
\hline Wavelength & Intensity & Wave No. & Term combination & Zeeman effect \\
\hline $\begin{array}{l}3308.15 \\
3307.02 \\
3306.95 \\
3304.73 \\
3301.21\end{array}$ & $\begin{array}{r}18 \\
50 \\
15 \\
5 \\
15\end{array}$ & $\begin{array}{l}30219.71 \\
30230.03 \\
30230.67 \\
30250.98 \\
30283.23\end{array}$ & $\begin{array}{c}c^{2} \mathrm{G}_{31 / 2}-y^{2} \mathrm{G}_{31 / 2}^{\circ} \\
b^{4} \mathrm{G}_{41 / 2}-z^{4} \mathrm{H}_{51 / 2}^{\circ} \\
c^{2} \mathrm{~F}_{31 / 2}-y^{2} \mathrm{G}_{41 / 2}^{\circ} \\
b^{2} \mathrm{G}_{31 / 2}-z^{2} \mathrm{~F}_{31 / 2}^{0} \\
c^{2} \mathrm{G}_{41 / 2}-y^{2} \mathrm{G}_{41 / 2}^{\circ}\end{array}$ & $\begin{array}{l}(0.27) 0.95 \dagger \\
(0.00 w D) 0.96 A \\
(0.00) 1.38 \dagger \\
(0.00) 1.10\end{array}$ \\
\hline $\begin{array}{l}3295.42 \\
3294.95 \\
3291.75 \\
3291.23\end{array}$ & $\begin{array}{r}50 \\
6 \\
40 \\
6\end{array}$ & $\begin{array}{l}\text { 30336. } 44 \\
\text { 30340. } 75 \\
\text { 30370. } 26 \\
\text { 30375. } 06\end{array}$ & $\begin{array}{l}b^{4} \mathrm{G}_{51 / 2}-z{ }^{4} \mathrm{H}_{61 / 2}^{\circ} \\
d^{2} \mathrm{D}_{11 / 2}-x^{2} \mathrm{P}_{11 / 2}^{\circ} \\
a^{2} \mathrm{P}_{01 / 2}-z^{2} \mathrm{~S}_{01 / 2}^{\circ}\end{array}$ & $\begin{array}{l}(0.00 w D) 1.02 A \\
(0.64) 1.32 \\
(0.00) 1.41 \dagger\end{array}$ \\
\hline 3288.04 & 15 & 30404.52 & $\begin{array}{l}d^{2} \mathrm{D}_{11 / 2}-x^{2} \mathrm{P}_{01 / 2}^{\circ} \\
a^{2} \mathrm{H}_{51 / 2}-z^{4} \mathrm{I}_{41 / 2}^{\circ}\end{array}$ & $(\mathbf{0 . 1 3}, 0.47,0.81,1.14,1.48) \ldots .1 .62,1.96,2.29$ \\
\hline $\begin{array}{l}3286.34 \\
3285.95 \\
3283.04 \\
3279.54 \\
3278.78\end{array}$ & $\begin{array}{r}1 \\
20 \\
20 \\
5 \\
2\end{array}$ & $\begin{array}{l}30420.25 \\
30423.85 \\
30450.83 \\
30483.33 \\
30490.39\end{array}$ & $\begin{array}{c}b^{2} \mathrm{D}_{11 / 2}-w^{4} \mathrm{D}_{01 / 2}^{\circ} \\
c^{2} \mathrm{G}_{31 / 2}-y^{2} \mathrm{G}_{41 / 2}^{\circ 1 / 2} \\
b^{2} \mathrm{I}_{51 / 2}-x^{4} \mathrm{G}_{41 / 2}^{\circ} \\
b^{2} \mathrm{G}_{31 / 2}-y^{4} \mathrm{H}_{41 / 2}^{\circ} \\
c^{4} \mathrm{D}_{31 / 2}-z^{2} \mathrm{~F}_{31 / 2}^{\circ}\end{array}$ & $\begin{array}{l}(0.00 w D) 1.56 B \\
(0.00 w) 0.52 A \dagger \\
(0.00) 1.11 \dagger\end{array}$ \\
\hline $\begin{array}{l}3276.24 \\
3275.91 \\
3273.19 \\
3272.87 \\
3272.73\end{array}$ & $\begin{array}{c}1 \\
10 \\
3 w \\
4 \\
1\end{array}$ & $\begin{array}{l}30514.02 \\
30517.10 \\
30542.46 \\
30545.45 \\
30546.76\end{array}$ & $\begin{array}{c}b^{2} \mathrm{D}_{21 / 2}-w^{4} \mathrm{D}_{11 / 2}^{\circ} \\
c^{2} \mathrm{~F}_{31 / 2}-y^{2} \mathrm{H}_{41 / 2}^{\circ} \\
d^{2} \mathrm{~F}_{21 / 2}-v^{2} \mathrm{~F}_{21 / 2}^{\circ} \\
b^{2} \mathrm{~S}_{01 / 2}^{\circ}-y^{2} \mathrm{P}_{01 / 2}^{\circ}\end{array}$ & $\begin{array}{l}(0.00) 1.05 \dagger \\
(0.00) 0.81 \dagger\end{array}$ \\
\hline $\begin{array}{l}\text { 3271. } 03 \\
3270.13\end{array}$ & $\begin{array}{r}1 \\
40\end{array}$ & $\begin{array}{l}\text { 30562. } 63 \\
30571.04\end{array}$ & $\begin{array}{l}d^{2} \mathrm{D}_{11 / 2}-x^{2} \mathrm{D}_{21 / 2}^{\circ} \\
a^{2} \mathrm{H}_{51 / 2}-z^{4} \mathrm{G}_{41 / 2}^{2}\end{array}$ & $(0.00 d ?) 1.00 \mathrm{~A}$ \\
\hline 3269. 76 & 15 & 30574.50 & $\begin{array}{l}c^{2} \mathrm{~F}_{21 / 2}-x^{4} \mathrm{G}_{21 / 2}^{0} \\
c^{2} \mathrm{G}_{41 / 2}-y^{2} \mathrm{H}_{51 / 2}^{1}\end{array}$ & \\
\hline $\begin{array}{l}3269.10 \\
3268.47\end{array}$ & $\begin{array}{l}30 \\
10+g\end{array}$ & $\begin{array}{l}30580.67 \\
30586.57\end{array}$ & $d^{2} \mathrm{D}_{21 / 2}-x^{2} \mathrm{D}_{21 / 2}^{01 / 2}$ & $\begin{array}{l}(0.00) 1.19 \\
(0.65) . . . ? \dagger\end{array}$ \\
\hline 3266.25 & 8 & 30607.35 & $\left\{\begin{array}{l}b^{2} \mathrm{G}_{41 / 2}-y^{4} \mathrm{H}_{51 / 2}^{\circ} \\
a^{2} \mathrm{H}_{51 / 2}-z^{4} \mathrm{I}_{51 / 2}^{\circ}\end{array}\right.$ & $(0.00) 1.17$ \\
\hline $\begin{array}{l}3264.26 \\
3261.88 \\
3261.54\end{array}$ & $\begin{array}{r}35 \\
4 \\
4\end{array}$ & $\begin{array}{l}30626.01 \\
30648.36 \\
30651.55\end{array}$ & $a^{2} \mathrm{H}_{41 / 2}^{51 / 2}-z^{4} \mathrm{G}_{31 / 2}^{\circ}$ & $(0.00 w D) 0.69 A$ \\
\hline 3258. 76 & 30 & 30677.70 & $\left\{\begin{array}{l}b^{2} \mathrm{~S}_{61 / 2}^{0}-y^{2} \mathrm{P}_{11 / 2}^{\circ} \\
b^{2} \mathrm{I}_{6 ! 12}-x^{4} \mathrm{G}_{51 / 2}^{\circ}\end{array}\right.$ & $\left(0.00 w_{3} D\right) 0.63 A$ \\
\hline $\begin{array}{l}3258.00 \\
3255.60 \\
3255.30 \\
3252.49 \\
3250.78\end{array}$ & $\begin{array}{r}3 \\
3 \\
15 \\
25 \\
10\end{array}$ & $\begin{array}{l}30684.86 \\
30707.48 \\
30710.31 \\
30736.84 \\
30753.01\end{array}$ & $\begin{array}{l}c^{2} \mathrm{~F}_{21 / 2}-x^{4} \mathrm{G}_{31 / 2}^{\circ} \\
c^{2} \mathrm{~F}_{31 / 2}-y^{2} \mathrm{~F}_{21 / 2}^{21 / 2} \\
c^{2} \mathrm{G}_{31 / 2}-y^{2} \mathrm{H}_{41 / 2}^{\circ} \\
d^{2} \mathrm{D}_{11 / 2}-x^{2} \mathrm{D}_{11 / 2}^{\circ} \\
a^{2} \mathrm{H}_{41 / 2}-z^{4} \mathrm{G}_{41 / 2}^{\circ}\end{array}$ & $\begin{array}{l}(0.00) 1.18 \dagger \\
(0.07) 0.88 \\
(0.94) . . . ? \dagger\end{array}$ \\
\hline $\begin{array}{l}3250.59 \\
3249.51 \\
3247.33 \\
3247.00 \\
3245.29\end{array}$ & $\begin{array}{r}1 \\
12 \\
8 \\
4 \\
5\end{array}$ & $\begin{array}{l}30754.80 \\
30765.03 \\
30785.68 \\
30788.81 \\
30805.03\end{array}$ & $\begin{array}{c}d^{2} \mathrm{D}_{21 / 2}-x^{2} \mathrm{D}_{11 / 2}^{\circ} \\
d^{2} \mathrm{~F}_{31 / 2}-v^{2} \mathrm{~F}_{31 / 2}^{\circ} \\
b^{2} \mathrm{~F}_{21 / 2}-y^{4} \mathrm{P}_{11 / 2}^{\circ} \\
a^{2} \mathrm{H}_{41 / 2}^{\circ}-z^{4} \mathrm{I}_{51 / 2}^{\circ} \\
a^{2} \mathrm{H}_{51 / 2}^{\circ}-z^{4} \mathrm{I}_{61 / 2}^{\circ}\end{array}$ & $\begin{array}{l}(0.00) 1.15 \\
(?) 0.31 A \dagger\end{array}$ \\
\hline $\begin{array}{l}3241.98 \\
3241.37 \\
3240.06 \\
3238.76 \\
3238.51\end{array}$ & $\begin{array}{r}2 \\
4 \\
7 \\
50 \\
10\end{array}$ & $\begin{array}{l}30836.48 \\
30842.28 \\
30854.75 \\
30867.13 \\
30869.52\end{array}$ & $\begin{array}{l}c^{2} \mathrm{~F}_{21 / 2}-y^{2} \mathrm{~F}_{21 / 2}^{\circ} \\
c^{2} \mathrm{G}_{41 / 2}-x^{4} \mathrm{G}_{41 / 2}^{01} \\
a^{2} \mathrm{H}_{51 / 2}-z^{2} \mathrm{G}_{41 / 2}^{\circ} \\
b^{4} \mathrm{~F}_{31 / 2}-z^{4} \mathrm{H}_{41 / 2}^{\circ}\end{array}$ & $\begin{array}{l}(0.31) 1.10 \dagger \\
(0.00 d ?) 0.96 A\end{array}$ \\
\hline 3235. 24 & 4 & 30900. 72 & $\begin{array}{l}c^{2} \mathrm{G}_{31 / 2}-y^{2} \mathrm{~F}_{21 / 2}^{\circ} \\
a^{2} \mathrm{P}_{11}-y^{4} \mathrm{P}_{02}^{\circ}\end{array}$ & \\
\hline $\begin{array}{l}\text { 3234. } 06 \\
3232.38 \\
3231.63 \\
3230.83\end{array}$ & $\begin{array}{r}50 \\
2 \\
8 \\
2\end{array}$ & $\begin{array}{l}\text { 30911. } 99 \\
\text { 30928. } 06 \\
\text { 30935. } 24 \\
\text { 30942. } 90\end{array}$ & $\begin{array}{l}a^{2} \mathrm{H}_{41 / 2}-z^{2} \mathrm{G}_{31 / 2}^{00} \\
b^{4} \mathrm{G}_{31 / 2}-y^{4} \mathrm{D}_{21 / 2}^{\circ} \\
b^{2} \mathrm{G}_{41 / 2}-x^{4} \mathrm{~F}_{41 / 2}^{\circ} \\
b^{2} \mathrm{G}_{41 / 2}-x^{4} \mathrm{~F}_{31 / 2}^{\circ}\end{array}$ & $\begin{array}{l}(0.00) 0.92 \\
(0.93) \cdot . ?+\end{array}$ \\
\hline $\begin{array}{l}3229.88 \\
3229.38 \\
3227.48 \\
3226.35 \\
3225.44\end{array}$ & $\begin{array}{r}10 \\
8 \\
3 \\
4 \\
8\end{array}$ & $\begin{array}{l}30952.00 \\
30956.79 \\
30975.01 \\
30985.86 \\
30994.60\end{array}$ & $\begin{array}{l}c^{4} \mathrm{D}_{01 / 2}-x{ }^{4} \mathrm{~F}_{11 / 2}^{0} \\
b^{4} \mathrm{~F}_{11 / 2}-y^{4} \mathrm{D}_{01 / 2}^{0} \\
c^{2} \mathrm{~F}_{31 / 2}-y^{2} \mathrm{~F}_{31 / 2}^{0} \\
c^{4} \mathrm{D}_{11 / 2}-x^{4} \mathrm{~F}_{11 / 2}^{0} \\
b^{4} \mathrm{~F}_{41 / 2}-z^{4} \mathrm{H}_{51 / 2}^{0}\end{array}$ & $\begin{array}{l}(0.29) 0.29, \mathbf{0 . 9 2} \\
(0.00) 0.84\end{array}$ \\
\hline
\end{tabular}


TABLE 1. Wavelengths of $\mathrm{Cr}$ II in air-Continued

\begin{tabular}{|c|c|c|c|c|}
\hline Wavelength & Intensity & Wave No. & Term combination & Zeeman effect \\
\hline $\begin{array}{l}\text { 3225. } 38 \\
3221.39 \\
3219.79 \\
3219.13 \\
3217.40\end{array}$ & $\begin{array}{r}12 \\
1 \\
10 \\
18 \\
50\end{array}$ & $\begin{array}{l}\text { 30995. } 18 \\
\text { 31033. } 57 \\
\text { 31048. } 99 \\
\text { 31055. } 36 \\
\text { 31072. } 01\end{array}$ & $\begin{array}{l}c^{2} \mathrm{G}_{31 / 2}-x^{4} \mathrm{G}_{41 / 2}^{\circ} \\
c^{4} \mathrm{D}_{21 / 2}-x^{4} \mathrm{~F}_{11 / 2}^{\circ} \\
a^{2} \mathrm{H}_{41 / 2}-z^{2} \mathrm{G}_{41 / 2}^{\circ} \\
c^{2} \mathrm{G}_{41 / 2}-x^{4} \mathrm{G}_{51 / 2}^{\circ} \\
a^{4} \mathrm{G}_{21 / 2}-z^{4} \mathrm{~F}_{11 / 2}^{0}\end{array}$ & $\begin{array}{l}(0.00 w D) 1.14 w \\
(0.76, \mathbf{0 . 9 8}) \dot{1} \dot{B} \cdot 1.28,1.47 \\
(0.00 w D) 1.37 \dot{B} \\
(0.00) 0.80 B \dagger\end{array}$ \\
\hline $\begin{array}{l}3216.55 \\
3213.46 \\
3212.90 \\
3212.52 \\
3211.49\end{array}$ & $\begin{array}{r}20 \\
3 \\
18 \\
20 \\
12\end{array}$ & $\begin{array}{l}31080.27 \\
31110.15 \\
31115.57 \\
31119.25 \\
31129.23\end{array}$ & $\begin{array}{l}b^{2} \mathrm{~F}_{21 / 2}-z^{2} \mathrm{D}_{11 / 2}^{\circ} \\
c^{2} \mathrm{~F}_{21 / 2}^{\circ}-y^{2} \mathrm{~F}_{31 / 2}^{\circ} \\
c^{4} \mathrm{D}_{11 / 2}^{\circ}-x^{4} \mathrm{~F}_{21 / 2}^{\circ} \\
b^{2} \mathrm{~F}_{31 / 2}-y^{4} \mathrm{P}_{21 / 2}^{\circ} \\
e^{2} \mathrm{G}_{31 / 2}-v^{2} \mathrm{G}_{31 / 2}^{\circ}\end{array}$ & $\begin{array}{l}(0.00) 0.84 \\
(0.00) 0.83 \dagger \\
(\mathbf{0 . 1 9}, 0.57,0.92) \quad \mathbf{0 . 1 9}, 0.57,0.92 \\
(0.00 w) 0.84 \dagger\end{array}$ \\
\hline $\begin{array}{l}\text { 3209. } 19 \\
3208.60 \\
3208.01 \\
3205.35 \\
3205.11\end{array}$ & $\begin{array}{r}50 \\
20 \\
8 \\
2 \\
25\end{array}$ & $\begin{array}{l}\text { 31151. } 57 \\
31157.29 \\
31163.00 \\
31188.85 \\
31191.20\end{array}$ & $\begin{array}{l}a^{4} \mathrm{G}_{31 / 2}-z^{4} \mathrm{~F}_{21 / 2}^{\circ} \\
a^{4} \mathrm{G}_{21 / 2}-z^{4} \mathrm{~F}_{21 / 2}^{\circ} \\
c^{4} \mathrm{D}_{21 / 2}-x{ }^{4} \mathrm{~F}_{21 / 2}^{\circ} \\
c^{4} \mathrm{D}_{21 / 2}-x{ }^{4} \mathrm{~F}_{31 / 2}^{\circ}\end{array}$ & $\begin{array}{l}\mathrm{P}-\mathrm{B} \\
\mathrm{P}-\mathrm{B} \\
(0.81) 1.39 \dagger \\
(\boldsymbol{\theta . 0 0}, 0.19,0.35) \mathbf{0 . 8 7}, 1.19\end{array}$ \\
\hline $\begin{array}{l}\text { 3203. } 53 \\
3202.51 \\
\text { 3202. } 47 \\
\text { 3201. } 26 \\
\text { 3200. } 44\end{array}$ & $\begin{array}{r}15 \\
15 \\
7 \\
25 \\
10\end{array}$ & $\begin{array}{l}31206.58 \\
31216.52 \\
31216.91 \\
31228.70 \\
31236.71\end{array}$ & $\begin{array}{l}b^{4} \mathbf{F}_{21 / 2}-y^{4} \mathrm{D}_{11 / 2}^{\circ} \\
b^{2} \mathrm{D}_{21 / 2}-x^{2} \mathrm{~F}_{31 / 2}^{\circ} \\
b^{4} \mathrm{~F}_{11 / 2}-y^{4} \mathrm{D}_{11 / 2}^{\circ} \\
c^{4} \mathrm{D}_{31 / 2}-x^{4} \mathrm{~F}_{41 / 2}^{\circ} \\
c^{4} \mathrm{D}_{31 / 2}-x^{4} \mathrm{~F}_{31 / 2}^{\circ}\end{array}$ & $\begin{array}{l}(0.00 w) \mathbf{0 . 7 6}, 0.95 \dagger \\
(0.00 w) 1.07 A \\
(\mathbf{0 . 0 7}, 0.21,0.35) \quad \mathbf{0 . 8 5}, 1.07,1.22,1.36,1.51,1.63 \\
(0.71 B) 1.18 C^{\dagger}\end{array}$ \\
\hline $\begin{array}{l}3199.86 \\
3198.74 \\
3198.00 \\
3197.08 \\
3196.93\end{array}$ & $\begin{array}{r}10 \\
2 \\
15 \\
75 \\
20\end{array}$ & $\begin{array}{l}31242.37 \\
31253.30 \\
31260.54 \\
31269.48 \\
31270.99\end{array}$ & $\begin{array}{l}c^{4} \mathrm{D}_{01 / 2}-y^{2} \mathrm{D}_{11 / 2}^{\circ} \\
e^{2} \mathrm{G}_{41 / 2}-v^{2} \mathrm{G}_{41 / 2}^{\circ} \\
a^{4} \mathrm{G}_{41 / 2}-z^{4} \mathrm{~F}_{31 / 2}^{\circ} \\
a^{4} \mathrm{G}_{31 / 2}-z^{4} \mathrm{~F}_{31 / 2}^{\circ 1 / 2}\end{array}$ & $\begin{array}{l}(0.31) 0.31,0.91 \\
(0.00) 1.16 \dagger \\
\mathrm{P}-\mathrm{B} \\
\mathrm{P}-\mathrm{B}\end{array}$ \\
\hline $\begin{array}{l}3196.39 \\
3196.35 \\
3194.62 \\
3193.41 \\
3190.68\end{array}$ & $\begin{array}{r}5 \\
3 \\
10 \\
2 \\
6\end{array}$ & $\begin{array}{l}31276.31 \\
31276.67 \\
31293.61 \\
31305.47 \\
31332.25\end{array}$ & $\begin{array}{l}c^{4} \mathrm{D}_{11 / 2}-y^{2} \mathrm{D}_{11 / 2}^{\circ} \\
a^{4} \mathrm{G}_{21 / 2}-z^{4} \mathrm{~F}_{31 / 2}^{\circ} \\
a^{2} \mathrm{P}_{11 / 2}-z^{2} \mathrm{D}_{11 / 2}^{\circ} \\
b^{4} \mathrm{G}_{41 / 2}-y^{4} \mathrm{D}_{31 / 2}^{\circ} \\
b^{2} \mathrm{D}_{11 / 2}-w^{4} \mathrm{~F}_{21 / 2}^{0}\end{array}$ & $\begin{array}{l}(0.24, \mathbf{0 . 6 7}) 0.67, \mathbf{1 . 1 1}, 1.55 \\
(0.00) 1.13 \dagger\end{array}$ \\
\hline $\begin{array}{l}3189.85 \\
3186.75 \\
3184.36 \\
3183.33 \\
3181.42\end{array}$ & $\begin{array}{l}12 \\
18 \\
15 \\
40 \\
20\end{array}$ & $\begin{array}{l}31340.41 \\
31370.89 \\
31394.43 \\
31404.59 \\
31423.41\end{array}$ & $\begin{array}{c}b^{2} \mathrm{G}_{41 / 2}-y^{2} \mathrm{G}_{31 / 2}^{\circ} \\
a^{2} \mathrm{P}_{11 / 2}-y^{4} \mathrm{P}_{21 / 2}^{\circ} \\
b^{2} \mathrm{G}_{31 / 2}-y^{2} \mathrm{G}_{31 / 2}^{\circ} \\
b^{2} \mathrm{~F}_{31 / 2}-z^{2} \mathrm{D}_{21 / 2}^{\circ} \\
a^{4} \mathrm{G}_{41 / 2}-z^{4} \mathrm{~F}_{41 / 2}^{0}\end{array}$ & $\begin{array}{l}(\mathbf{0 . 0 0}, 0.21,0.46,0.65) . \dot{1} \cdot 1.84 \\
(\mathbf{0 . 0 9}, 0.28) 1.41,1.61, \mathbf{1 . 7 8} \\
(0.00) 0.90 \\
(\mathbf{0 . 0 0} 0.16,0.30) 0.84 A \\
\mathrm{P}-\mathrm{B}\end{array}$ \\
\hline $\begin{array}{l}3180.70 \\
3179.46 \\
3178.80 \\
3177.90 \\
3177.50\end{array}$ & $\begin{array}{r}75 \\
8 \\
7 \\
1 \\
1\end{array}$ & $\begin{array}{l}31430.51 \\
31442.82 \\
31449.34 \\
31458.25 \\
31462.21\end{array}$ & $\begin{array}{l}a^{4} \mathrm{G}_{51 / 2}-z^{4} \mathbf{F}_{41 / 2}^{\circ} \\
b^{2} \mathbf{F}_{21 / 2}-z^{2} \mathrm{D}_{21 / 2}^{\circ} \\
b^{2} \mathrm{D}_{11 / 2}-x^{2} \mathbf{F}_{21 / 2}^{\circ} \\
a^{2} \mathbf{F}_{21 / 2}-y^{4} \mathrm{D}_{11 / 2}^{\circ}\end{array}$ & $\begin{array}{l}\mathrm{P}-\mathrm{B} \\
(0.62, \mathbf{0 . 9 4}) 1.25 \\
\left(0.00 w_{3} D\right) 1.05 w_{2}\end{array}$ \\
\hline $\begin{array}{l}3176.60 \\
3173.93 \\
3173.58 \\
3172.08 \\
3170.71\end{array}$ & $\begin{array}{l}4 \\
2 w \\
15 \\
40 \\
2\end{array}$ & $\begin{array}{l}31471.12 \\
31497.60 \\
31501.07 \\
31515.97 \\
31529.58\end{array}$ & $\begin{array}{l}b^{2} \mathrm{D}_{11 / 2}-x^{4} \mathrm{P}_{21 / 2}^{\circ} \\
b^{2} \mathrm{~F}_{21 / 2}-z^{2} \mathrm{P}_{11 / 2}^{\circ} \\
a^{2} \mathrm{P}_{11 / 2}-z^{2} \mathrm{P}_{01 / 2}^{\circ}\end{array}$ & $\begin{array}{l}(\mathbf{0 . 1 7}, 0.50) \\
(0.27) \\
(0.07,1.60\end{array}$ \\
\hline $\begin{array}{l}3169.86 \\
3169.20 \\
3168.39 \\
3164.48 \\
3164.28\end{array}$ & $\begin{array}{l}2 w, l \\
25 \\
7 \\
1 \\
4\end{array}$ & $\begin{array}{l}31538.04 \\
31544.61 \\
31552.67 \\
31591.66 \\
31593.65\end{array}$ & $\begin{array}{l}b^{2} \mathrm{D}_{21 / 2}-x^{2} \mathrm{~F}_{21 / 2}^{\circ} \\
b^{2} \mathrm{G}_{41 / 2}^{\circ}-y^{2} \mathrm{G}_{41 / 2}^{\circ} \\
c^{4} \mathrm{D}_{11 / 2}-y^{2} \mathrm{D}_{21 / 2}^{\circ} \\
b^{4} \mathrm{~F}_{21 / 2}^{\circ}-y{ }^{4} \mathrm{D}_{21 / 2}^{\circ}\end{array}$ & $(0.00) 1.12$ \\
\hline $\begin{array}{l}3163.93 \\
3163.37 \\
3162.46 \\
3160.11 \\
3159.86\end{array}$ & $\begin{array}{r}10 \\
3 \\
10 \\
5 \\
3\end{array}$ & $\begin{array}{l}\text { 31597. } 15 \\
\text { 31602. } 74 \\
\text { 31611. } 83 \\
\text { 31635. } 34 \\
\text { 31637. } 84\end{array}$ & $\begin{array}{l}a^{2} \mathrm{P}_{01 / 2}-y^{4} \mathrm{P}_{01 / 2}^{\circ} \\
a^{2} \mathrm{G}_{41 / 2}-x^{4} \mathrm{D}_{31 / 2}^{\circ} \\
b^{4} \mathrm{~F}_{31 / 2}-y^{4} \mathrm{D}_{21 / 2}^{\circ} \\
b^{4} \mathrm{G}_{31 / 2}-z^{4} \mathrm{G}_{21 / 2}^{\circ} \\
b^{4} \mathrm{G}_{41 / 2}-z^{4} \mathrm{G}_{31 / 2}^{\circ}\end{array}$ & $\begin{array}{l}(0.93) 1.62 \\
(0.00 w) 0.98 A \dagger \\
(\mathbf{0 . 2 1}, 0.62,1.01) 1.28,1.61, \mathbf{2 . 0 0}\end{array}$ \\
\hline $\begin{array}{l}3159.10 \\
3158.03 \\
3157.52 \\
3154.10 \\
3154.04\end{array}$ & $\begin{array}{r}7 \\
10 \\
2 \\
3 \\
3\end{array}$ & $\begin{array}{l}31645.45 \\
31656.17 \\
31661.29 \\
31695.62 \\
31696.22\end{array}$ & $\begin{array}{l}a^{4} \mathrm{D}_{31 / 2}-z^{4} \mathrm{~F}_{21 / 2}^{0} \\
a^{2} \mathrm{P}_{11 / 2}-z^{2} \mathrm{D}_{21 / 2}^{\circ} \\
b^{2} \mathrm{H}_{51 / 2}-y^{4} \mathrm{G}_{51 / 2} \\
a^{2} \mathrm{P}_{01 / 2}-y^{4} \mathrm{P}_{11 / 2}^{\circ} \\
b^{4} \mathrm{G}_{31 / 2}-x^{4} \mathrm{I}_{41 / 2}^{0}\end{array}$ & 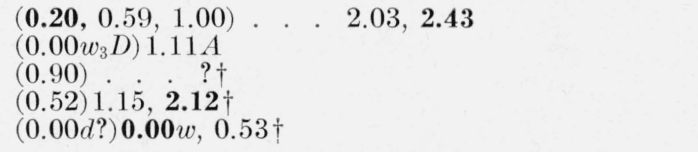 \\
\hline
\end{tabular}


TABLE 1. Wavelengths of $\mathrm{Cr}$ II in air-Continued

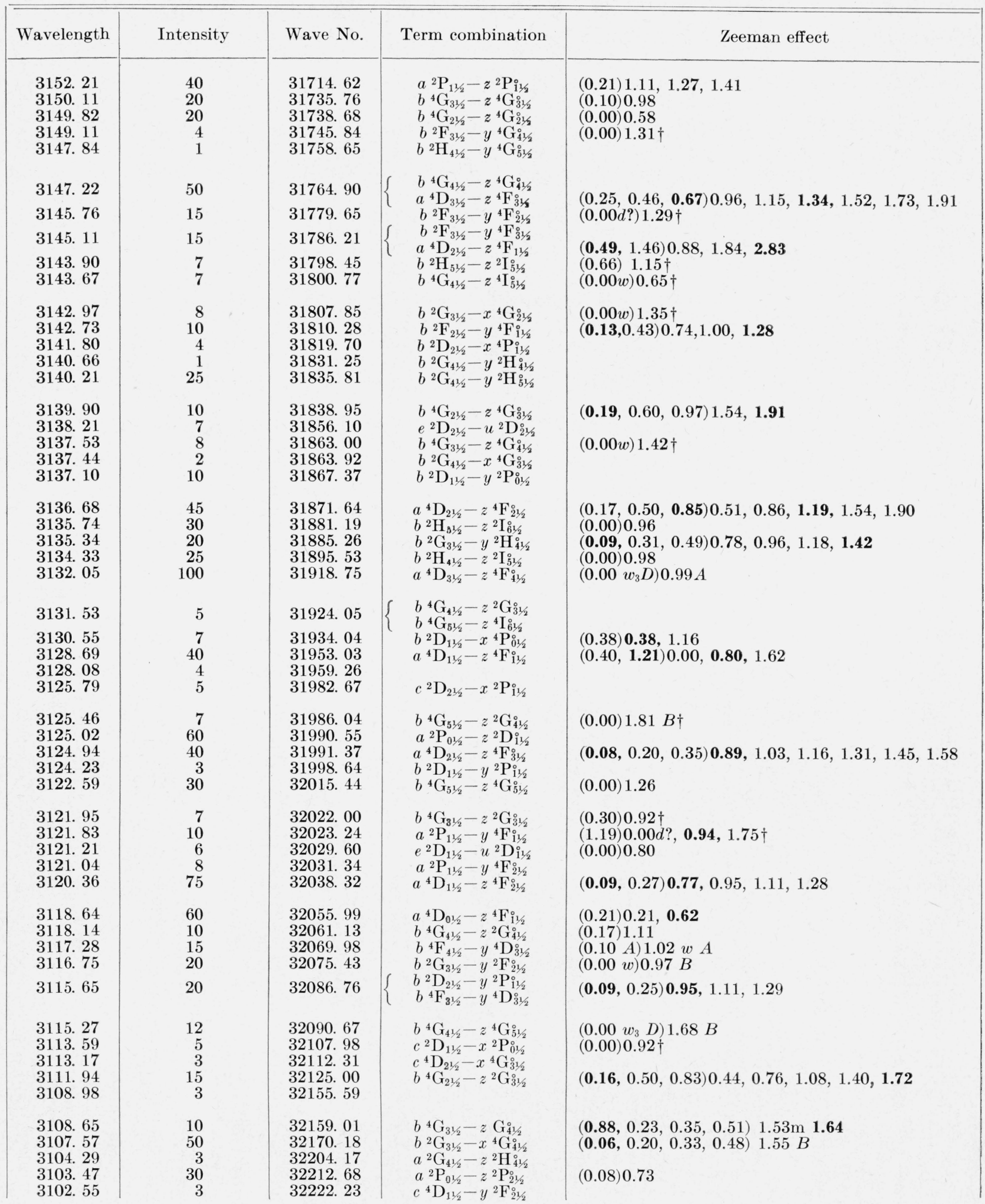


TABLE 1. Wavelengths of $\mathrm{Cr}$ II in air-Continued

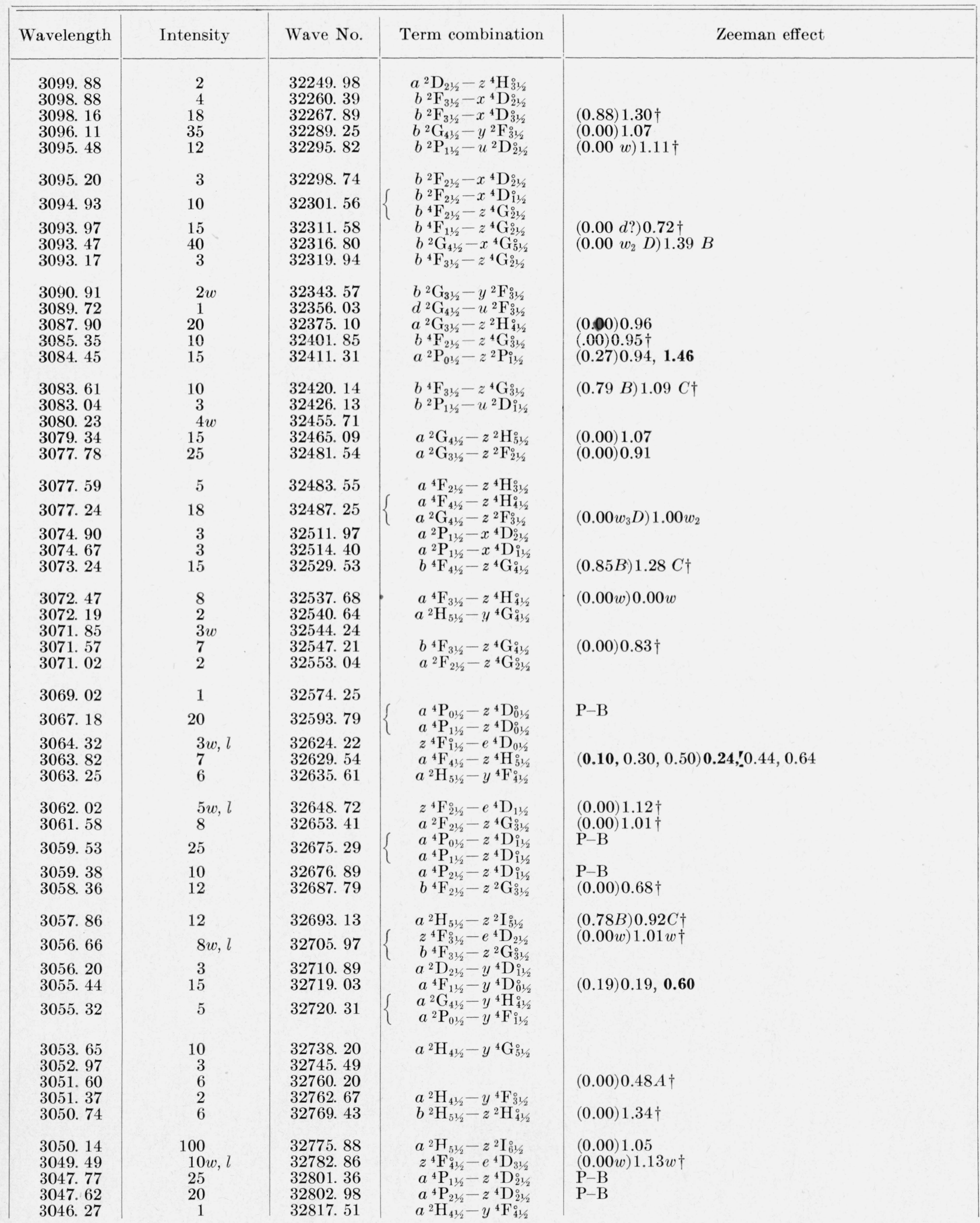


TABle 1. Wavelengths of $\mathrm{Cr}$ II in air-Continued

\begin{tabular}{|c|c|c|c|c|}
\hline Wavelength & Intensity & Wave No. & Term combination & Zeeman effect \\
\hline 3045. 62 & 3 & 32824.52 & \multirow{5}{*}{$\begin{array}{l}c^{2} \mathrm{G}_{41 / 2}-x^{2} \mathrm{G}_{31 / 2}^{\circ} \\
z^{4} \mathrm{~F}_{21 / 2}^{\circ}-e^{4} \mathrm{D}_{21 / 2}^{\circ} \\
b^{4} \mathrm{~F}_{41 / 2}-z^{2} \mathrm{G}_{41 / 2}^{\circ} \\
c^{2} \mathbf{F}_{31 / 2}-x^{2} \mathrm{G}_{41 / 2}^{\circ} \\
b^{4} \mathbf{F}_{31 / 2}-z^{2} \mathrm{G}_{41 / 2}^{\circ} \\
b^{4} \mathbf{F}_{41 / 2}-z^{4} \mathrm{G}_{51 / 2}^{0}\end{array}$} & \multirow[b]{2}{*}{$(0.36 B) 1.18 C \dagger$} \\
\hline 3045.52 & $4 l$ & 32825.49 & & \\
\hline 3044. 23 & 10 & 32839.51 & & \multirow{3}{*}{$\begin{array}{l}\left(0.00 w_{2}\right) 1.30 w_{1} \\
(0.00) 0.91 \dagger \\
(0.12) 1.12\end{array}$} \\
\hline 3043. 89 & 18 & 32843. 17 & & \\
\hline 3042. 79 & 25 & 32855.04 & & \\
\hline 3041. 73 & 50 & 32866. 49 & $b^{2} \mathrm{H}_{41 / 2}-z^{2} \mathrm{H}_{41 / 2}^{\circ}$ & \multirow{5}{*}{$\begin{array}{l}(0.00) 0.93 \\
(0.00) 0.97 \\
(0.00) 1.00 \dagger \\
(0.00) 1.19\end{array}$} \\
\hline 3040.91 & 70 & 32875.36 & $a^{2} \mathrm{H}_{41 / 2}^{41 / 2}-z^{2} \mathrm{I}_{51 / 2}^{\circ}$ & \\
\hline 3040.18 & $8 d ?$ & 32883. 25 & & \\
\hline 3039. 32 & 4 & 32892.55 & $c^{2} \mathrm{G}_{41 / 2}-x^{2} \mathrm{G}_{41 / 2}^{\circ}$ & \\
\hline 3038. 80 & 4 & 32898. 18 & $a^{2} \mathrm{G}_{41 / 2}-y{ }^{4} \mathrm{H}_{51 / 2}^{\circ}$ & \\
\hline 3038.51 & 4 & 32901. 32 & $a^{2} \mathrm{~F}_{31 / 2}-z^{4} \mathrm{G}_{31 / 2}^{\circ}$ & \multirow{5}{*}{$\begin{array}{l}(0.00) 0.96 \dagger \\
(0.00) 0.91 \\
(\mathbf{0 . 0 9}, 0.26) 0.78\end{array}$} \\
\hline 3038. 04 & 8 & 32906. 42 & $c^{2} \mathbf{F}_{21 / 2}-x^{2} \mathrm{G}_{31 / 2}^{\circ}$ & \\
\hline 3034. 99 & 20 & 32939. 48 & (6) $\quad a^{2} \mathrm{~F}_{21 / 2}-z^{2} \mathrm{G}_{31 / 2}$ & \\
\hline $\begin{array}{l}3034.54 \\
3034.06\end{array}$ & 25 & 32944. 37 & $a^{4} \mathrm{~F}_{21 / 2}-y{ }^{4} \mathrm{D}_{11 / 2}^{\circ}$ & \\
\hline & & 58578.58 & ${ }^{a} 11 / 2 \sim 11 / 2$ & \\
\hline 3032. 94 & 50 & 32961. 74 & $a^{4} \mathrm{P}_{21 / 2}-z^{4} \mathrm{D}_{31 / 2}^{\circ}$ & \multirow{5}{*}{$\begin{array}{l}(0.00) 0.86 \\
(0.00) 1.09\end{array}$} \\
\hline 3032.65 & 4 & 32964. 90 & $c^{2} \mathrm{G}_{31 / 2}-x^{2} \mathrm{G}_{31 / 2}^{\circ}$ & \\
\hline 3031. 63 & 3 & 32975. 98 & $b^{2} \mathrm{~F}_{31 / 2}-z^{2} \mathrm{~F}_{21 / 2}^{0}$ & \\
\hline 3028.12 & 75 & 33014. 21 & $b^{2} \mathrm{~F}_{21 / 2}-z^{2} \mathrm{~F}_{21 / 2}^{\circ}$ & \\
\hline 3026. 86 & 20 & 33027. 95 & $a^{2} \mathrm{~F}_{31 / 2}-z^{4} \mathrm{G}_{41 / 2}^{0}$ & \\
\hline 3026.64 & 100 & 33030.35 & $b{ }^{2} \mathrm{H}_{51 / 2}-z^{2} \mathrm{H}_{51 / 2}^{\circ}$ & \multirow[t]{5}{*}{$(0.00) 1.10$} \\
\hline 3026. 38 & 7 & 33033. 19 & $c^{2} \mathrm{G}_{31 / 2}-x^{2} \mathrm{G}_{41 / 2}^{\circ}$ & \\
\hline 3024. 90 & 3 & 33049.35 & \multirow{3}{*}{$\begin{array}{l}z^{6} \mathrm{D}_{11 / 2}^{\circ}-e^{6} \mathrm{D}_{01 / 2} \\
b^{2} \mathrm{H}_{41 / 2}-z^{2} \mathrm{H}_{51 / 2}^{0} \\
b^{2} \mathbf{F}_{31 / 2}-z^{2} \mathrm{~F}_{31 / 2}^{\circ}\end{array}$} & \\
\hline 3017. 78 & $10 w$ & 33127.32 & & \\
\hline 3015.51 & 50 & 33152. 26 & & \\
\hline 3012. 47 & 5 & 33185. 71 & \multirow{5}{*}{$\begin{array}{l}a^{2} \mathrm{~F}_{31 / 2}-z^{2} \mathrm{G}_{31 / 2}^{\circ} \\
b^{2} \mathrm{~F}_{21 / 2}-z^{2} \mathrm{~F}_{31 / 2}^{\circ} \\
b^{4} \mathrm{P}_{21 / 2}-y^{4} \mathrm{D}_{11 / 2}^{\circ} \\
z^{6} \mathrm{D}_{01 / 2}^{\circ}-e^{6} \mathrm{D}_{01 / 2} \\
z^{6} \mathrm{D}_{41 / 2}^{\circ}-e^{6} \mathrm{D}_{31 / 2}^{\circ}\end{array}$} & . \\
\hline 3012.33 & 3 & 33187. 25 & & \multirow{4}{*}{$(\mathbf{0 . 2 0}, 0.59) 1.38,1.80, \mathbf{2 . 1 8}$} \\
\hline 3012.01 & 2 & 33190. 78 & & \\
\hline 3011.42 & 7 & 33197.28 & & \\
\hline 3010. 92 & $4 w, l$ & 33202. 79 & & \\
\hline 3010. 64 & 10 & 33205.88 & $b^{4} \mathrm{G}_{31 / 2}-y^{4} \mathrm{P}_{21 / 2}^{\circ}$ & $(0.00) 1.32$ \\
\hline 3008. 67 & 4 & 33227. 62 & $a^{2} \mathrm{P}_{11 / 2}-z^{2} \mathrm{~F}_{21 / 2}^{0}$ & \\
\hline 3008. 30 & 6 & 33231. 71 & $b^{4} \mathrm{G}_{21 / 2}-z^{2} \mathrm{D}_{11 / 2}^{\circ}$ & $(0.00 w) 0.00 W \dagger$ \\
\hline 3007. 98 & $6 w, l$ & 33235. 25 & $\begin{array}{l}b^{2} \mathrm{~F}^{31 / 2}-y^{4} \mathrm{H}_{31 / 2}^{1}{ }^{1} \\
z^{6} \mathrm{D}_{31 / 2}^{\circ}-e^{6} \mathrm{D}_{21 / 2}^{\circ}\end{array}$ & \\
\hline 3004.77 & $2 w, l$ & 33270.75 & $z^{6} \mathrm{D}_{01 / 2}^{\circ / 2}-e^{6} \mathrm{D}_{11 / 2}$ & \\
\hline 3004. 47 & 3 & 33274.07 & $b^{2} \mathbf{F}_{21 / 2}-y{ }^{4} \mathrm{H}_{31 / 2}^{\circ}$ & $(0.00) 0.40 \dagger$ \\
\hline 3003. 92 & & 33280.16 & $a^{4} \mathbf{F}_{31 / 2}-y^{4} \mathrm{D}_{21 / 2}^{0}$ & $(\mathbf{0 . 0 7}, 0.22,0.35) 0.88,1.02,1.18$ \\
\hline 3000.65 & $2 w, l$ & 33316.43 & $z^{6} \mathrm{D}_{11 / 2}^{0}-e{ }^{6} \mathrm{D}_{21 / 2}$ & \\
\hline 2999. 96 & 25 & 33324. 09 & $a^{2} \mathrm{~F}_{31 / 2}-z^{2} \mathrm{G}_{41 / 2}^{0}$ & $(0.00) 0.95$ \\
\hline 2999. 30 & 8 & 33331. 42 & $a^{4} \mathrm{~F}_{21 / 2}-y{ }^{4} \mathrm{D}_{21 / 2}^{\circ}$ & $(0.86 \mathrm{~B}) 1.38 \mathrm{C}^{\dagger}$ \\
\hline 2999. 00 & $1 w, l$ & 33334.66 & $z^{6} \mathrm{D}_{21 / 2}^{\circ}-e{ }^{6} \mathrm{D}_{31 / 2}$ & \\
\hline 2994. 74 & 20 & 33382. 17 & $a{ }^{4} \mathrm{H}_{41 / 2}-z^{4} \mathrm{H}_{31 / 2}^{0}$ & $(0.00 W D) 2.09 B \dagger$ \\
\hline $\begin{array}{l}2993.54 \\
2992.96\end{array}$ & $\begin{array}{rl}1 & w, l \\
10 w, l\end{array}$ & $\begin{array}{l}33395.55 \\
33402.03\end{array}$ & $\begin{array}{l}z^{6} \mathrm{D}_{31 / 2}^{\circ}-e^{6} \mathrm{D}_{31 / 2} \\
z^{6} \mathrm{D}_{41 / 3}^{0}-e^{6} \mathrm{D}_{41 / 1}\end{array}$ & \\
\hline 2992. 59 & 7 & 33406. 16 & $b^{2} \mathrm{~S}_{01 / 2}-x^{2} \mathrm{P}_{11 / 2}$ & \\
\hline 2992. 42 & 10 & 33408. 05 & $a{ }^{4} \mathrm{H}_{51 / 2}-z{ }^{4} \mathrm{H}_{41 / 2}^{\circ}$ & $(\mathbf{0 . 0 0}, 0.23,0.42,0.59,0.73) 1.74, \mathbf{1 . 8 5}$ \\
\hline 2989. 18 & 70 & 33444. 26 & $a{ }^{4} \mathrm{H}_{31 / 2}-z^{4} \mathrm{H}_{31 / 2}^{0}$ & $(0.00) 0.68$ \\
\hline 2988. 04 & 12 & 33457. 02 & $a{ }^{4} \mathrm{H}_{61 / 2}-z^{4} \mathrm{H}_{51 / 2}^{\circ}$ & $\left(0.00 w_{2} D\right) 1.73 B$ \\
\hline $\begin{array}{l}2987.52 \\
2086.87\end{array}$ & $\begin{array}{l}3 \\
8\end{array}$ & 33462.84 & $b{ }^{2} \mathrm{H}_{51 / 2}-y{ }^{4} \mathrm{H}_{51 / 2}^{\circ}$ & \\
\hline 2986.87 & 8 & 33470.13 & $b^{2} \mathrm{~S}_{01 / 2}-x^{2} \mathrm{P}_{01 / 2}$ & $(0.00) 1.281$ \\
\hline 2985. 32 & 75 & 33487.50 & $a{ }^{4} \mathrm{H}_{41 / 2}-z{ }^{4} \mathrm{H}_{41 / 2}$ & $(0.00) 0.98$ \\
\hline 2985. 01 & 7 & 33490. 98 & $b^{4} \mathrm{G}_{31 / 2}-z^{2} \mathrm{D}_{21 / 2}$ & \\
\hline $\begin{array}{l}2984.69 \\
2982.69\end{array}$ & 10 & 33494.57 & $b{ }^{4} \mathrm{P}_{11 / 2}-y{ }^{4} \mathrm{D}_{01 / 2}^{\circ}$ & $\begin{array}{l}(0.87) 0.87,2.56 \dagger \\
0.00) 1.27 \dagger\end{array}$ \\
\hline $\begin{array}{l}2982.09 \\
2979.73\end{array}$ & 80 & $\begin{array}{l}33517.03 \\
33550.32\end{array}$ & $a^{4} \mathrm{H}_{51 / 2}-z^{4} \mathrm{H}_{51 / 2}^{\circ}$ & $(0.00) 1.13$ \\
\hline 2977. 65 & 2 & 33573. 76 & $a^{2} \mathrm{D}_{21 / 2}-y{ }^{4} \mathrm{D}_{31 / 2}^{\circ}$ & \\
\hline 2976. 70 & 35 & 33584. 47 & $b{ }^{4} \mathrm{P}_{21 / 2}-y^{4} \mathrm{D}_{21 / 2}$ & $(0.34, \mathbf{0 . 5 9}) 1.03,1.27, \mathbf{1 . 5 0}, 1.73,1.89$ \\
\hline $\begin{array}{l}2975.80 \\
297483\end{array}$ & $\begin{array}{l}4 w \\
4 w, l\end{array}$ & $\begin{array}{l}33594.63 \\
33605.58\end{array}$ & $z^{6} \mathrm{D}_{31 / 2}^{\circ}-e^{6} \mathrm{D}_{41 / 2}$ & \\
\hline 2973. 10 & & $\begin{array}{l}\text { 33605. } 58 \\
33625.14\end{array}$ & $a^{2} \mathrm{D}_{11 / 2}-z^{4} \mathrm{G}_{21 / 2}^{\circ}$ & $(\mathbf{0 . 1 1}, 0.31) \mathbf{0 . 2 6}, 0.50,0.79$ \\
\hline
\end{tabular}


TABLE 1. Wavelengths of Cr II in air-Continued

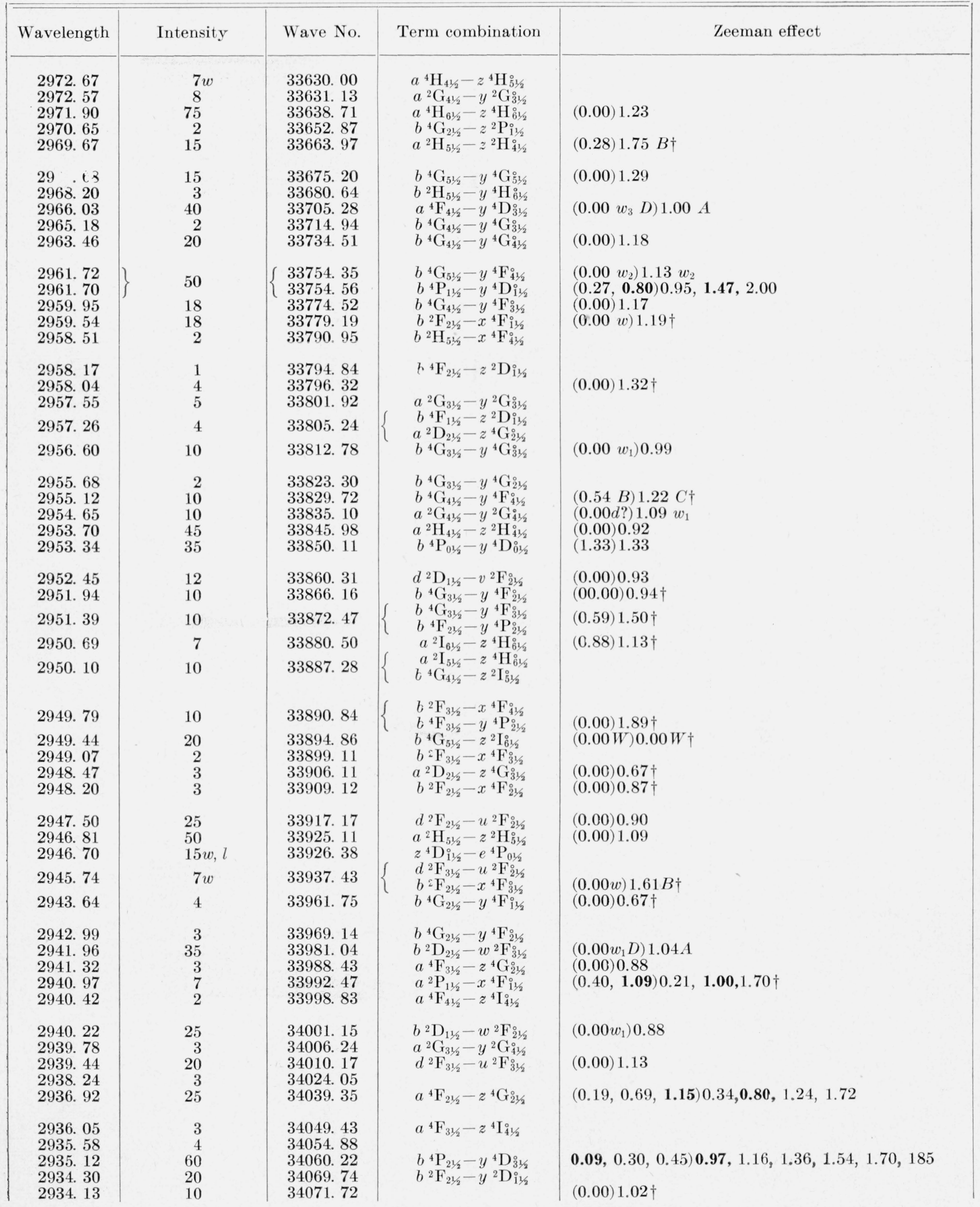


TABLE 1. Wavelengths of $\mathrm{Cr}$ II in air-Continued

\begin{tabular}{|c|c|c|c|c|}
\hline Wavelength & Intensity & Wave No. & Term combination & Zeeman effect \\
\hline $\begin{array}{l}2933.95 \\
2933.60\end{array}$ & $\begin{array}{l}35 \\
12\end{array}$ & $\begin{array}{l}\text { 34073. } 80 \\
34077.87\end{array}$ & $\begin{array}{l}a^{4} \mathrm{~F}_{11 / 2}-z^{4} \mathrm{G}_{21 / 2}^{\circ} \\
d^{2} \mathrm{D}_{21 / 2}-v^{2} \mathrm{~F}_{31 / 2}^{\circ}\end{array}$ & $\begin{array}{l}(\mathbf{0 . 0 7}, 0.26) 0.33,0.49,0.64, \mathbf{0 . 8 3} \\
(0.00) 1.13 \dagger\end{array}$ \\
\hline $\begin{array}{l}\text { 2932. } 69 \\
\text { 2931. } 07 \\
2930.83\end{array}$ & $\begin{array}{r}30 \\
4 \\
35\end{array}$ & $\begin{array}{l}\text { 34088. } 45 \\
\text { 34107. } 29 \\
\text { 34110. } 07\end{array}$ & $\begin{array}{l}a^{4} \mathrm{~F}_{31 / 2}-z^{4} \mathrm{G}_{31 / 2}^{\circ} \\
a^{2} \mathrm{H}_{41 / 2}-z^{2} \mathrm{H}_{51 / 2}^{\circ} \\
b^{4} \mathrm{P}_{01 / 2}-y^{4} \mathrm{D}_{11 / 2}^{\circ}\end{array}$ & 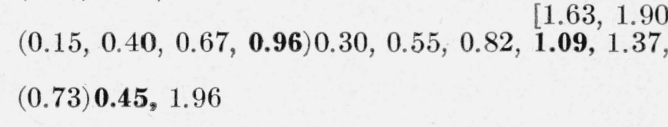 \\
\hline $\begin{array}{l}\text { 2929. } 78 \\
\text { 2929. } 44 \\
\text { 2929. } 18\end{array}$ & $\begin{array}{r}4 \\
18 \\
2\end{array}$ & $\begin{array}{l}\text { 34122. } 30 \\
\text { 34126. } 26 \\
34129.29\end{array}$ & $\begin{array}{l}a^{2} \mathrm{P}_{11 / 2}-x^{4} \mathrm{~F}_{21 / 2}^{\circ} \\
a^{2} \mathrm{G}_{41 / 2}-y^{2} \mathrm{H}_{51 / 2}^{\circ} \\
a^{2} \mathrm{H}_{41 / 2}-z^{2} \mathrm{~F}_{31 / 2}^{\circ}\end{array}$ & $\begin{array}{l}(0.28) 0.99 \dagger \\
(0.00 w) 1.51 \dagger\end{array}$ \\
\hline 2928. 32 & 50 & 34139.31 & $\begin{array}{l}b^{2} \mathrm{G}_{31 / 2}-x^{2} \mathrm{G}_{31 / 2}^{\circ} \\
a^{4} \mathrm{~F}_{21 / 2}-z^{4} \mathrm{G}_{31 / 2}^{1}\end{array}$ & $(0.00) 0.90 \dagger$ \\
\hline 2928. 12 & 40 & 34141. 65 & ${ }^{4} \mathrm{P}_{11 / 2}^{21 / 2}-y{ }^{4} \mathrm{D}_{21 / 2}^{8}$ & $(0.19,0.55) 0.82,1.21,1.57$ \\
\hline 2927. 09 & 50 & 34153. 66 & $b^{2} \mathrm{G}_{41 / 2}-x^{2} \mathrm{G}_{41 / 2}^{\circ}$ & $(0.00) 1.10$ \\
\hline 2926.15 & 18 & 34164.62 & $a^{4} \mathrm{~F}_{41 / 2}-z^{4} \mathrm{G}_{41 / 2}^{\circ 1 / 2}$ & $\begin{array}{l}(0.28,0.51,0.73, \mathbf{0 . 9 5}) 0.82,1.03,1.25,1.43,1.66 \\
\quad 1.88,2.09\end{array}$ \\
\hline $\begin{array}{l}2925.90 \\
2925.22\end{array}$ & $\begin{array}{l}3 \\
3\end{array}$ & $\begin{array}{l}34167.55 \\
34175.48\end{array}$ & $b^{4} \mathrm{~F}_{11 / 2}-z^{2} \mathrm{D}_{21 / 2}^{\circ}$ & \\
\hline 2924. 86 & $\begin{array}{l}3 \\
2\end{array}$ & $\begin{array}{l}34175.48 \\
34179.69\end{array}$ & $\begin{array}{l}b^{4} \mathrm{~F}_{31 / 2}-z^{2} \mathrm{D}_{21 / 2}^{2} \\
a^{2} \mathrm{H}_{51 / 2}-y^{4} \mathrm{H}_{41 / 2}^{\circ}\end{array}$ & $(0.00) 1.22 \uparrow$ \\
\hline 2923. 80 & 8 & 34192. 08 & $a^{2} \mathrm{D}_{21 / 2}-z^{2} \mathrm{G}_{31 / 2}^{\circ}$ & \\
\hline 2923. 67 & 40 & 34193. 60 & $b^{2} I_{51 / 2}-y^{2} I_{51 / 2}^{0}$ & $(0.26) 0.97 \dagger$ \\
\hline 2923. 46 & 30 & 34196. 06 & $b^{2} \mathrm{I}_{51 / 2}-y^{2} \mathrm{I}_{61 / 2}$ & $(0.00 w) 1.45 \dagger$ \\
\hline $\begin{array}{l}\text { 2922. } 46 \\
2921.81\end{array}$ & $\begin{array}{r}5 \\
40\end{array}$ & $\begin{array}{l}34207.76 \\
34215.37\end{array}$ & $\begin{array}{l}b^{2} \mathrm{G}_{31 / 2}-x^{2} \mathrm{G}_{41 / 2}^{0} \\
a^{4} \mathrm{~F}_{31 / 2}-z^{4} \mathrm{G}_{41 / 2}^{\circ}\end{array}$ & $(0.00 w) 0.85 A \dagger$ \\
\hline 2921. 23 & 50 & 34222. 17 & $b^{2} I_{61 / 6}-y^{2} I_{61 / 6}^{\circ}$ & $(0.28) 1.06 \dagger$ \\
\hline 2921. 10 & 5 & 34223. 69 & $0.161 / 2-y-161 / 2$ & $(0.00) 0.32$ \\
\hline 2920. 90 & 4 & 34226.03 & & \\
\hline 2919. 93 & $2 w$ & 34237. 40 & $c^{2} \mathrm{~F}_{31 / 2}-x^{2} \mathrm{~F}_{31 / 2}^{\circ}$ & \\
\hline 2918. 93 & $1 w, l$ & 34249. 13 & $z^{6} \mathrm{P}_{31 / 2}^{0}-e^{6} \mathrm{D}_{21 / 2}$ & \\
\hline 2918. 29 & 3 & 34256. 64 & $b^{4} \mathrm{G}_{41 / 2}-x^{4} \mathrm{D}_{31 / 2}^{\circ}$ & \\
\hline 2917. 40 & $1 w, l$ & 34267.09 & ${ }^{6} \mathrm{P}_{0}$ & \\
\hline $\begin{array}{l}\text { 2916. } 94 \\
2916.07\end{array}$ & $\begin{array}{l}2 w, l \\
10\end{array}$ & $\begin{array}{l}34272.49 \\
34282.72\end{array}$ & $\begin{array}{l}z^{6} \mathrm{P}_{21 / 2}^{0}-e^{6} \mathrm{D}_{11 / 2} \\
a^{2} \mathrm{P}_{11 / 2}-y^{2} \mathrm{D}_{116}^{0}\end{array}$ & $(0.40,1.10) 0.21, \mathbf{0 . 9 9}, 1.72 \dagger$ \\
\hline 2915. 46 & 30 & 34289.89 & $c^{2} \mathrm{G}_{41 / 2}^{11 / 2}-x^{2} \mathrm{~F}_{31 / 2}^{13 / 2}$ & $(0.00) 1.06 \dagger$ \\
\hline 2915. 28 & 15 & 34292. 01 & $a^{2} \mathrm{G}_{31 / 2}-y^{2} \mathrm{H}_{41 / 2}^{\circ}$ & $(0.00) 1.46$ \\
\hline 2915. 22 & 10 & 34292. 71 & $b^{2} \mathrm{H}_{41 / 2}-y^{2} \mathrm{G}_{31 / 2}$ & $(0.00) 1.33$ \\
\hline 2914. 38 & 2 & 34302.60 & $a^{2} \mathrm{~S}_{01 / 2}-x^{1} \mathrm{P}_{11 / 2}$ & $(\bullet .00) 1.52$ \\
\hline $\begin{array}{l}2913.50 \\
2912.53\end{array}$ & 10 & $\begin{array}{l}34312.96 \\
34324.38\end{array}$ & $a^{4} \mathrm{~F}_{41 / 2}-z^{2} \mathrm{G}_{31 / 2}^{\circ}$ & $(0.00) 0.72$ \\
\hline & & & 政 & ( 000$) 008+$ \\
\hline 2911. 69 & 35 & 34334. 29 & $\begin{array}{l}b^{2} \mathbf{F}_{21 / 2}-y^{2} \mathrm{G}_{31 / 2}^{0} \\
b^{4} \mathrm{G}_{31 / 2}-x^{4} \mathrm{D}_{21 / 2}^{0}\end{array}$ & $(0.00) 0.98 \dagger$ \\
\hline 2910. 64 & 30 & 34346. 67 & $b^{2} \mathrm{~F}_{31 / 2}-y^{2} \mathrm{D}_{21 / 2}^{23 / 2}$ & $\left(0.00 w_{1} D\right) 1.06 \mathrm{~A}$ \\
\hline $\begin{array}{l}2909.13 \\
2908.29\end{array}$ & $\begin{array}{l}2 w, l \\
10\end{array}$ & $\begin{array}{l}34364.50 \\
34374.42\end{array}$ & $z^{6} \mathrm{P}_{11 / 2}-e^{6} \mathrm{D}_{11 / 2}$ & \\
\hline $\begin{array}{l}2908.29 \\
2907.00\end{array}$ & $4 w$ & $\begin{array}{l}34374.42 \\
34389.68\end{array}$ & $\begin{array}{l}a^{4} \mathrm{~F}_{31 / 2}-z^{2} \mathrm{G}_{31 / 2}^{8} \\
z^{6} \mathrm{P}_{21 / 2}^{\circ}-e^{6} \mathrm{D}_{21 / 2}^{0}\end{array}$ & $(0.78,1.14) 0.70,1.08,1.38,1.73,2.02$ \\
\hline 2906. 76 & 2 & 34392.52 & $b^{4} \mathrm{P}_{21 / 2}-z^{4} \mathrm{G}_{31 / 2}^{\circ}$ & \\
\hline 2906. 17 & 10 & 34399.50 & $b^{2} \mathrm{H}_{51 / 2}^{2 / 2}-y^{2} \mathrm{G}_{41 / 2}^{0}$ & $(0.00) 1.50 \dagger$ \\
\hline $\begin{array}{l}2905.57 \\
2903.97\end{array}$ & $\begin{array}{r}3 \\
20\end{array}$ & $\begin{array}{l}34406.60 \\
34425,56\end{array}$ & $\begin{array}{r}a^{2} \mathrm{G}_{41 / 2}-x^{4} \mathrm{G}_{41 / 2} \\
a^{4} \mathrm{~F}_{21}-z^{2} \mathrm{G}^{2}\end{array}$ & $(0.00 \mathrm{D}) 0.58 \mathrm{~A}$ \\
\hline 2903.58 & $\begin{array}{l}20 \\
15\end{array}$ & $\begin{array}{l}34425.56 \\
34430.18\end{array}$ & $a^{4} \mathrm{~F}_{21 / 2}-z^{2} \mathrm{G}_{31 / 2}^{1}$ & $\begin{array}{l}(0.00) \\
(0.00) 1.80\end{array}$ \\
\hline 2902.86 & 10 & 34438. 72 & $a^{2} \mathbf{S}_{01 / 2}-y{ }^{2} \mathrm{P}_{01 / 2}^{\circ}$ & (0.12) 1.88 \\
\hline 2902. 60 & 7 & 34441. 81 & $c^{2} \mathbf{F}_{31 / 2}-w^{4} \mathbf{F}_{21 / 2}^{2}$ & $(0.00) 1.20$ \\
\hline 2901.00 & 12 & 34460.80 & $a^{4} \mathrm{~F}_{41 / 2}-z^{2} \mathrm{G}_{41 / 2}$ & $(0.76 B) 1.27 C \dagger$ \\
\hline 2900.50 & 4 & 34466. 74 & $a^{2} \mathrm{~F}_{21 / 2}-z^{2} \mathrm{P}_{11 / 2}$ & \\
\hline 2899. 48 & 35 & 34478.86 & $b{ }^{4} \mathrm{~F}_{21 / 2}-y{ }^{4} \mathrm{G}_{31 / 2}^{0}$ & $(0.00) 0.99 \dagger$ \\
\hline 2899.15 & 25 & 34482. 79 & $a^{2} \mathrm{G}_{31 / 2}-y^{2} \mathrm{~F}_{21 / 2}^{\circ}$ & $(0.00) 0.97 \dagger$ \\
\hline 2898. 53 & 50 & 34490. 16 & $a^{4} \mathrm{~F}_{41 / 2}-z^{4} \mathrm{G}_{51 / 2}^{\circ}$ & $\left(0.00 w_{3} D\right) 1.02 A$ \\
\hline $\begin{array}{l}2897.82 \\
2897.73\end{array}$ & $\begin{array}{l}10 \\
20\end{array}$ & $\begin{array}{l}34498.61 \\
34499.68\end{array}$ & $\begin{array}{l}b^{4} \mathrm{~F}_{41 / 2}-y^{4} \mathrm{G}_{41 / 2} \\
b^{4} \mathrm{~F}_{112}-y^{4} \mathrm{G}^{0}\end{array}$ & $(0.00) 1.08$ \\
\hline 2897. 67 & 30 & 34500. 40 & $b^{2} \mathrm{~F}_{31 / 2}-y^{2} \mathrm{G}_{41 / 2}$ & $(0.00) 1.10 \dagger$ \\
\hline
\end{tabular}


TABLE 1. Wavelengths of $\mathrm{Cr}$ II in air-Continued

\begin{tabular}{|c|c|c|c|c|}
\hline Wavelength & Intensity & Wave No. & Term combinatiọn & Zeeman effect. \\
\hline 2897.24 & 10 & 34505.52 & $\begin{array}{l}b^{2} \mathrm{I}_{61 / 2}-x^{2} \mathrm{H}_{51 / 2}^{\circ} \\
a^{2} \mathrm{~S}_{01-x}-x \mathrm{P}^{0}{ }^{0} 10\end{array}$ & $(0.00 w) 1.17, \mathbf{1 . 9 1} \dagger$ \\
\hline 2896. 74 & 35 & 34511.47 & $a^{4} \mathrm{~F}_{31 / 2}^{01 / 2}-z^{2} \mathrm{G}_{41 / 2}^{0 / 2}$ & $\left(0.00 w_{3} D\right) 0.82 A$ \\
\hline 2896.45 & 40 & 34514. 93 & $\begin{array}{l}b^{2} \mathrm{I}_{51 / 2}-z^{2} \mathrm{~K}_{61 / 2}^{0} \\
b^{4} \mathrm{~F}_{41 / 2}-y^{4} \mathrm{G}_{51 / 1}^{\circ}\end{array}$ & $\left(0.00 w_{3} D\right) 0.99,1.26$ \\
\hline 2896. 31 & 30 & 34516.60 & $b^{4} \mathrm{~F}_{31 / 2}-y^{4} \mathrm{G}_{41 / 2}$ & $(0.00) 1.07 \dagger$ \\
\hline 2895. 66 & 5 & 34524.35 & $b^{4} \mathrm{~F}_{21 / 2}-y^{4} \mathrm{~F}_{11 / 2}^{0}$ & $(0.38) 1.76 \dagger$ \\
\hline 2895. 02 & 18 & 34531. 98 & $\left\{\begin{array}{l}c^{2} \mathrm{~F}_{21 / 2}-w^{4} \mathrm{~F}_{11 / 2}^{\circ} \\
b^{4} \mathrm{~F}_{21}-y^{4} \mathrm{~F}_{21}^{\circ}\end{array}\right.$ & $(0.00) 1.04 \dagger$ \\
\hline 2894. 81 & 18 & 34534. 48 & $b^{4} \mathrm{~F}_{11 / 2}-y^{4} \mathrm{~F}_{11 / 2}^{01 / 2}$ & $(0.00) 0.47 \dagger$ \\
\hline $\begin{array}{l}2894.40 \\
2894.24\end{array}$ & $\begin{array}{l}10 \\
25\end{array}$ & $\begin{array}{l}34539.38 \\
34541.28\end{array}$ & $b^{4} \mathrm{~F}_{41 / 2}-y^{4} \mathrm{~F}_{31 / 2}^{1}$ & \\
\hline $\begin{array}{l}2894.24 \\
2893.50\end{array}$ & $4 w, l$ & $\begin{array}{l}34541.28 \\
34550.12\end{array}$ & $\left\{\begin{array}{l}z^{6} \mathrm{P}_{21 / 2}-e^{6} \mathrm{D}_{31 / 2} \\
b^{4} \mathrm{~F}_{31 / 2}-y^{4} \mathrm{~F}_{21 / 2}^{0}\end{array}\right.$ & $(0.00 \mathrm{w}) 1.70 \dagger$ \\
\hline 2892.95 & 20 & 34556. 68 & $b^{4} \mathrm{~F}_{31 / 2}-y{ }^{4} \overline{\mathrm{F}}_{31 / 2}^{\circ}$ & $(0.16) 1.25 \dagger$ \\
\hline 2892. 74 & 18 & 34559. 20 & 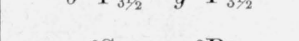 & $(0.00) 1.28 \dagger$ \\
\hline 2891.87 & 20 & 34569.59 & $a^{2} \mathbf{S}_{01 / 2}-y^{2} \mathbf{P}_{11 / 2}^{\circ}$ & $(0.33) \mathbf{1 . 0 4}, 1.76$ \\
\hline 2891. 40 & $\begin{array}{l}20 \\
20\end{array}$ & $\begin{array}{l}34575.21 \\
34577.60\end{array}$ & $a^{2} \mathrm{H}_{51 / 2}-y^{4} \mathrm{H}_{61 / 2}^{0}$ & $(0.00 w) 1.40 \dagger$ \\
\hline 2891.20 & & 34577.60 & $a^{2} \mathrm{G}_{31 / 2}-x^{4} \mathrm{G}_{41 / 2}^{0}$ & $(0.00 w) 1.36 \dagger$ \\
\hline 2891. 06 & 25 & 34579.27 & $a^{2} \mathrm{G}_{41 / 2}-y^{2} \mathrm{~F}_{31 / 2}^{\circ}$ & $\left(0.00 w_{1}\right) 1.03 w_{1}$ \\
\hline 2889. 82 & 25 & 34594.11 & $b^{4} \mathrm{~F}_{41 / 2}^{1 / 2}-y^{4} \mathrm{~F}_{41 / 2}^{\circ}$ & $(0.00) 1.32$ \\
\hline 2889.50 & 35 & 34597.94 & $a^{2} \mathbf{P}_{11 / 2}-y^{2} \mathbf{D}_{21 / 2}^{2}$ & $(0.00) 1.10^{\dagger}$ \\
\hline 2889. 19 & 35 & 34601. 66 & $a^{4} \mathrm{D}_{31 / 2}-z^{4} \mathrm{D}_{21 / 2}^{2}$ & $\left(0.00 w_{1}\right) 1.49$ \\
\hline 2888. 73 & 40 & 34607. 16 & $a^{2} \mathrm{G}_{41 / 2}^{0 / 2}-x^{4} \mathrm{G}_{51 / 2}^{0}$ & $(0.14 C) 1.34 B$ \\
\hline 2888. 33 & $2 w, l$ & 34611.98 & $z^{6} \mathrm{D}_{21 / 2}^{\circ}-e^{4} \mathrm{D}_{11 / 2}$ & \\
\hline 2887. 77 & 20 & 34618.67 & $c^{2} \mathrm{D}_{11 / 2}-w^{2} \mathrm{D}_{11 / 2}^{2}$ & $(0.00) 0.79$ \\
\hline 2886. 38 & 7 & 34635.34 & $c^{2} \mathrm{G}_{31 / 2}-w^{4} \mathrm{~F}_{21 / 2}^{0}$ & $(0.00) 0.96$ \\
\hline $\begin{array}{l}2885.29 \\
2884.98\end{array}$ & $\begin{array}{r}10 \\
2\end{array}$ & $\begin{array}{l}34648.42 \\
34652\end{array}$ & $b^{4} \mathrm{~F} u-z^{2} I_{0}$ & $(0.00) 1.12$ \\
\hline 2884.98 & 2 & 34652.15 & $0.14 \frac{1}{2}-2-151 / 2$ & \\
\hline 2884. 61 & 1 & 34656.59 & $a^{2} \mathrm{~F}_{31 / 2}-z^{2} \mathrm{D}_{21 / 2}^{\circ}$ & \\
\hline 2881. 91 & 45 & 34689.06 & $a^{2} \mathrm{P}_{01 / 2}-x^{4} \mathrm{~F}_{11 / 2}^{11 / 2}$ & $(0.00) 0.64 \dagger$ \\
\hline 2881. 86 & 55 & 34689.65 & $c^{2} \mathrm{D}_{21 / 2}-w^{2} \mathbf{D}_{21 / 2}^{2}$ & $(0.00) 1.18 \dagger$ \\
\hline 2880.86 & & 34701. 70 & $a{ }^{4} \mathrm{D}_{21 / 2}-z^{4} \mathrm{D}_{11 / 2}^{\circ}$ & $(\mathbf{0 . 1 1}, 0.25) 1.26,1.46,1.64$ \\
\hline 2880.08 & $2 w, l$ & 34711.10 & & \\
\hline 2879.68 & $3 w, l$ & 34715. 92 & $z^{6} \mathrm{D}_{01 / 2}^{\circ}-e^{4} \mathrm{D}_{01 / 2}$ & \\
\hline 2879. 17 & & 34722.07 & $b^{4} \mathrm{P}_{11 / 2}^{01 / 2}-z^{2} \mathrm{~S}_{01 / 2}$ & $(0.00 d) 1.64 \dagger$ \\
\hline 2878.45 & 50 & 34730.75 & $a^{6} \mathrm{D}_{41 / 2}-z^{6} \mathrm{~F}_{31 / 2}^{0}$ & $(\mathbf{0 . 0 7}, 0.20,0.34,0.48) 1.52, \cdot 1.73,1.93,2.12$ \\
\hline $\begin{array}{l}2877.97 \\
2876.66\end{array}$ & $\begin{array}{l}60 \\
20\end{array}$ & $\begin{array}{l}34736.55 \\
3475236\end{array}$ & $a^{6} \mathrm{D}_{31 / 2}-z^{6} \mathrm{~F}_{21 / 2}^{\circ}$ & $(\mathbf{0 . 1 3}, 0.41,0.69) 1.17,1.45,173,2.00,2.27$ \\
\hline & & & 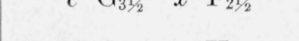 & $(0.00) 1.001$ \\
\hline 2876. 30 & 40 & 34756. 71 & $b^{2} \mathrm{I}_{61 / 2}-z^{2} \mathrm{~K}_{71 / 2}^{\circ}$ & (0.00) 1.01 \\
\hline 2876. 24 & 60 & 34757.44 & $a^{6} \mathrm{D}_{21 / 2}-z_{0} \mathrm{~F}_{11 / 2}$ & $(\mathbf{0 . 3 0}, 0.88) 1.37,1.95,2.56$ \\
\hline $\begin{array}{l}2875.97 \\
2875.03\end{array}$ & $\begin{array}{r}100 \\
30\end{array}$ & $\begin{array}{l}34760.70 \\
34772.07\end{array}$ & $\begin{array}{l}a^{4} \mathrm{D}_{31 / 2}-z^{4} \mathrm{D}_{31 / 2}^{\circ} \\
c^{2} \mathrm{G}_{31 / 2}-x^{2} \mathrm{H}_{41 / 2}^{0}\end{array}$ & $\begin{array}{l}(0.00) 1.43 \\
\left(0.00 w_{3} D\right) 0.68 A\end{array}$ \\
\hline 2874.51 & 10 & 34778. 36 & & $(0.00) 1.15 \dagger$ \\
\hline 2874. 07 & 8 & 34783. 68 & $b{ }^{2} \mathrm{H}_{41 / 2}-y^{2} \mathrm{H}_{41 / 2}^{\circ}$ & \\
\hline 2873. 81 & 50 & 34786.83 & $a^{4} \mathrm{D}_{11 / 2}-z^{4} \mathrm{D}_{01 / 2}$ & $(0.62) 0.62,1.78$ \\
\hline 2873. 46 & 65 & 34791. 06 & $\left\{\begin{array}{l}b^{2} \mathrm{D}_{11 / 2}-x^{2} \mathrm{P}_{01 / 2}^{0} \\
a^{6} \mathrm{D}_{11 / 2}-z^{6} \mathrm{~F}_{01 / 2}^{\circ}\end{array}\right.$ & $(1.26) 0.61,3.14$ \\
\hline $\begin{array}{l}\text { 2871. } 45 \\
2870.43\end{array}$ & $\begin{array}{r}20 \\
100\end{array}$ & $\begin{array}{l}\text { 34815. } 41 \\
\text { 34827. } 79\end{array}$ & $\begin{array}{l}b^{2} \mathrm{D}_{21 / 2}-x^{2} \mathrm{P}_{11 / 2}^{012} \\
a^{4} \mathrm{D}_{21 / 2}-z^{4} \mathrm{D}_{21 / 2}^{\circ}\end{array}$ & $\begin{array}{l}\left(0.00 w_{1}\right) 1.10 \\
(0.00) 1.38\end{array}$ \\
\hline 2869. 72 & $3 w, l$ & 34836. 41 & $z^{4} \mathrm{D}_{31 / 2}^{\circ}-f{ }^{4} \mathrm{D}_{31 / 2}$ & \\
\hline 2869. 61 & $3 w, l$ & 34837. 74 & $z^{4} \mathrm{D}_{11 / 2}^{31 / 2}-f^{4} \mathrm{D}_{11 / 2}^{31 / 2}$ & \\
\hline 2868. 63 & $4 w, l$ & 34849.64 & $\left\{\begin{array}{l}z^{4} \mathrm{D}_{21 / 2}^{\circ}-f^{4} \mathrm{D}_{21 / 2} \\
b^{4} \mathrm{P}_{11 / 2}-z^{4} \mathrm{G}^{0}{ }^{2} / 6\end{array}\right.$ & \\
\hline 2868. 47 & $2 w, l$ & 34851.59 & $z^{4} \mathrm{D}_{01 / 2}^{11 / 2}-f^{4} \mathrm{D}_{01 / 2}^{21 / 2}$ & \\
\hline 2867. 94 & $4 w$ & 34858. 03 & $\left\{\begin{array}{l}b^{2} \mathrm{~F}_{21 / 2}-x^{4} \mathrm{G}_{31 / 2}^{\circ} \\
b^{4} \mathrm{G}_{41 / 2}-z^{2} \mathrm{H}_{41 / 2}^{\circ 1 / 2}\end{array}\right.$ & \\
\hline 2867. 65 & 100 & 34861.55 & $a^{6} \mathrm{D}_{01 / 2}-z{ }^{6} \mathrm{~F}_{01 / 2}^{\circ}$ & $(2.00) 1.28$ \\
\hline 2867. 09 & 65 & 34868. 36 & $a^{4} \mathrm{D}_{11 / 2}-z^{4} \mathrm{D}_{11 / 2}^{\circ}$ & $(0.00) 1.16$ \\
\hline 2866. 72 & 100 & 34872. 86 & $a^{6} \mathrm{D}_{1 \%}-z^{6} \mathrm{~F}_{11 / 2}$ & $(0.40, \mathbf{1 . 1 8}) 0.70, \mathbf{1 . 5 3}, 2.29$ \\
\hline 2865. 87 & $\begin{array}{l}50 \\
20\end{array}$ & 34883.19 & $c^{2} \mathrm{G}_{41 / 2}-x^{2} \mathrm{H}_{51 / 2}^{\circ}$ & $(0.00) 0.87 \dagger$ \\
\hline 2865.65 & 20 & 34885.88 & $d^{2} \mathbf{F}_{21 / 2}-v^{2} \mathrm{G}_{31 / 2}^{\circ}$ & $(0.00) 0.96 \dagger$ \\
\hline
\end{tabular}


TABLE 1. Wavelengths of $\mathrm{Cr}$ II in air-Continued

\begin{tabular}{|c|c|c|c|c|}
\hline Wavelength & Intensity & Wave No. & Term combination & Zeeman effect \\
\hline $\begin{array}{l}2865.34 \\
2865.10\end{array}$ & $\begin{array}{r}30 \\
150\end{array}$ & $\begin{array}{l}34889.65 \\
34892.58\end{array}$ & $\begin{array}{l}a^{4} \mathrm{D}_{01 / 2}-z^{4} \mathrm{D}_{01 / 2}^{\circ} \\
a^{6} \mathrm{D}_{21 / 2}-z^{6} \mathrm{~F}_{21 / 2}^{\circ}\end{array}$ & $\begin{array}{l}(0.00) 0.00 \dagger \\
(0.16,0.50, \mathbf{0 . 8 8}) 1.14, \mathbf{1 . 5 0}, 1.84,2.18\end{array}$ \\
\hline $\begin{array}{l}2862.57 \\
2860.92 \\
2858.91\end{array}$ & $\begin{array}{r}125 \\
85 \\
75\end{array}$ & $\begin{array}{l}\text { 34923. } 41 \\
34943.55 \\
34968.12\end{array}$ & $\begin{array}{l}a^{6} \mathrm{D}_{31 / 2}-z^{6} \mathrm{~F}_{31 / 2}^{\circ} \\
a^{6} \mathrm{D}_{01 / 2}-z^{6} \mathrm{~F}_{11 / 2}^{31} \\
a^{6} \mathrm{D}_{41 / 2}-z^{6} \mathrm{~F}_{41 / 2}^{0}\end{array}$ & $\begin{array}{l}(0.27,0.49, \mathbf{0 . 6 9}) 0.92,1.11,1.30,1.50,1.70,1.89 \\
(1.13) 0.06,2.18 \\
(0.57 B) 1.49\end{array}$ \\
\hline $\begin{array}{l}2858.64 \\
2857.99 \\
2857.40 \\
2856.77 \\
2856.42\end{array}$ & $\begin{array}{r}30 \\
20 \\
40 \\
40 \\
4\end{array}$ & $\begin{array}{l}\text { 34971. } 42 \\
\text { 34979. } 38 \\
\text { 34986. } 60 \\
\text { 34994. } 31 \\
\text { 34998. } 60\end{array}$ & $\begin{array}{c}a^{4} \mathrm{D}_{01 / 2}-z^{4} \mathrm{D}_{11 / 2}^{\circ} \\
a^{2} \mathrm{P}_{01 / 2}-y^{2} \mathrm{D}_{11 / 2}^{\circ} \\
a^{4} \mathrm{D}_{21 / 2}-z^{4} \mathrm{D}_{31 / 2}^{\circ} \\
a^{4} \mathrm{D}_{11 / 2}-z^{4} \mathrm{D}_{21 / 2}^{\circ} \\
a^{4} \mathrm{H}_{41 / 2}-z^{4} \mathrm{I}_{41 / 2}^{0}\end{array}$ & $\begin{array}{l}(0.60) 0.60,1.80 \dagger \\
(0.00) 0.60 \dagger \\
(0.00) 1.52 \dagger \\
(0.00) 1.50 B \dagger\end{array}$ \\
\hline $\begin{array}{l}2856.32 \\
2855.67 \\
2855.43\end{array}$ & $\begin{array}{r}20 \\
100 \\
8\end{array}$ & $\begin{array}{l}\text { 34999. } 82 \\
\text { 35007. } 79 \\
\text { 35010. } 73\end{array}$ & $\begin{array}{l}a^{4} \mathrm{H}_{31 / 2}-z^{4} \mathrm{G}_{21 / 2}^{\circ} \\
a^{6} \mathrm{D}_{11 / 2}-z^{6} \mathrm{~F}_{21 / 2}^{\circ} \\
c^{4} \mathrm{D}_{01 / 2}-w^{4} \mathrm{D}_{01 / 2}^{\circ}\end{array}$ & $\begin{array}{l}\left(0.00 w_{1}\right) 0.94 \\
(\mathbf{0 . 3 0}, 0.84) \mathbf{0 . 5 3}, 1.07,1.58,2.15\end{array}$ \\
\hline 2855. 05 & 35 & 35015. 39 & $\left\{\begin{array}{l}b^{4} \mathrm{~F}_{21 / 2}-x^{4} \mathrm{D}_{11 / 2}^{\circ} \\
b^{4} \mathrm{~F}_{11 / 2}-x^{4} \mathrm{D}_{11 / 2} \\
b^{2} \mathrm{~F}-y^{2} \mathrm{~F}^{0}\end{array}\right.$ & $(0.00) 0.86 \dagger$ \\
\hline 2854.65 & 3 & 35020.30 & 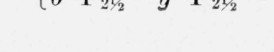 & \\
\hline $\begin{array}{l}2854.58 \\
2854.23 \\
2854.14 \\
2853.76 \\
2853.26\end{array}$ & $\begin{array}{l}5 \\
3 \\
20 w, d ? \\
8 \\
30\end{array}$ & $\begin{array}{l}\text { 35021. } 16 \\
\text { 35025. } 45 \\
\text { 35026. } 56 \\
\text { 35031. } 22 \\
\text { 35037. } 36\end{array}$ & $\begin{array}{c}b^{4} \mathrm{~F}_{41 / 2}-x^{4} \mathrm{D}_{31 / 2}^{\circ} \\
b^{4} \mathrm{~F}_{11 / 2}-x^{4} \mathrm{D}_{11 / 2}^{\circ} \\
b^{4} \mathrm{~F}_{31 / 2}-x^{4} \mathrm{D}_{21 / 2}^{\circ} \\
b^{2} \mathrm{D}_{21 / 2}-x^{2} \mathrm{D}_{21 / 2}^{2}\end{array}$ & $\begin{array}{l}(0.00) 1.29 \dagger \\
(0.00) 0.94 \dagger \\
(0.00) 1.22\end{array}$ \\
\hline $\begin{array}{l}2853.18 \\
2852.75 \\
2852.67 \\
2852.27 \\
2851.35\end{array}$ & $\begin{array}{r}30 \\
7 \\
20 \\
25 \\
60\end{array}$ & $\begin{array}{l}\text { 35038. } 34 \\
\text { 35043. } 62 \\
35044.61 \\
\text { 35049. } 52 \\
\text { 35060. } 83\end{array}$ & $\begin{array}{l}a^{4} \mathrm{H}_{41 / 2}-z^{4} \mathrm{G}_{31 / 2}^{\circ} \\
b^{4} \mathrm{G}_{51 / 2}-z^{2} \mathrm{H}_{01 / 2}^{\circ} \\
c^{4} \mathrm{D}_{11 / 2}-w^{4} \mathrm{D}_{01 / 2}^{0} \\
c^{4} \mathrm{D}_{11 / 2}-w^{4} \mathrm{D}_{11 / 2}^{\circ} \\
a^{4} \mathrm{H}_{31 / 2}-z^{4} \mathrm{I}_{41 / 2}^{\circ}\end{array}$ & $\begin{array}{l}(0.00) 1.00 \\
(0.00) 1.16 \dagger \\
\left(0.00 w_{3} D\right) 1.03 B\end{array}$ \\
\hline $\begin{array}{l}2850.72 \\
2850.29 \\
2849.83 \\
2849.33 \\
2848.40\end{array}$ & $\begin{array}{c}7 \\
3 \\
100 \\
18 \\
20 d ?\end{array}$ & $\begin{array}{l}\text { 35068. } 58 \\
35073.87 \\
35079.53 \\
\text { 35085. } 68 \\
\text { 35097. } 14\end{array}$ & $\begin{array}{l}b^{2} \mathrm{H}_{41 / 2}-x^{4} \mathrm{G}_{41 / 2}^{\circ} \\
c^{4} \mathrm{D}_{11 / 2}-w^{4} \mathrm{D}_{21 / 2}^{\circ} \\
a^{6} \mathrm{D}_{21 / 2}-z^{6} \mathrm{~F}_{31 / 2}^{2} \\
a^{4} \mathrm{H}_{51 / 2}-z^{4} \mathrm{G}_{41 / 2}^{\circ} \\
c^{4} \mathrm{D}_{21 / 2}-w^{4} \mathrm{D}_{11 / 2}^{\circ}\end{array}$ & $\begin{array}{l}(\mathbf{0 . 1 2}, 0.37,0.67) \mathbf{0 . 6 8}, 1.00,1.27,1.54,1.81,2.07 \\
(0.00) \\
1.14\end{array}$ \\
\hline $\begin{array}{l}2848.15 \\
2846.70\end{array}$ & 15 & $\begin{array}{l}\text { 35098. } 99 \\
35118.10\end{array}$ & $\begin{array}{l}a{ }^{4} \mathrm{H}_{31 / 2}-z^{4} \mathrm{G}_{31 / 2}^{0} \\
a^{2} \mathrm{D}_{11 / 2}-z^{2} \mathrm{D}_{11 / 2}^{\circ}\end{array}$ & $(0.18 B) 0.90 C$ \\
\hline 2846. 44 & 30 & 35121. 31 & $\left\{\begin{array}{l}c^{4} \mathrm{D}_{21 / 2}-w^{4} \mathrm{D}_{21 / 2}^{\circ} \\
a^{4} \mathrm{H}_{51 / 2}-z^{4} \mathrm{I}_{51 / 2}^{\circ}\end{array}\right.$ & $(0.00) 1.37$ \\
\hline $\begin{array}{l}\text { 2846. } 32 \\
2844.83\end{array}$ & $\begin{array}{r}25 \\
3\end{array}$ & $\begin{array}{l}\text { 35122. } 78 \\
\text { 35141. } 18\end{array}$ & $\begin{array}{l}b^{2} \mathrm{D}_{11 / 2}-x^{2} \mathrm{D}_{11 / 2}^{\circ} \\
b^{4} \mathrm{G}_{41 / 2}-z^{2} \mathrm{~F}_{31 / 2}^{\circ}\end{array}$ & $(0.05 B) 0.86 C$ \\
\hline $\begin{array}{l}2843.24 \\
2842.78 \\
2842.43 \\
2842.32 \\
2841.15\end{array}$ & $\begin{array}{r}100 \\
20 \\
5 \\
5 \\
2 w, l\end{array}$ & $\begin{array}{l}35160.83 \\
35166.52 \\
35170.85 \\
35172.21 \\
35186.69\end{array}$ & $\begin{array}{l}a^{6} \mathrm{D}_{31 / 2}-z^{6} \mathrm{~F}_{41 / 2}^{\circ} \\
c^{4} \mathrm{D}_{31 / 2}-w^{4} \mathrm{D}_{21 / 2}^{2} \\
c^{4} \mathrm{D}_{21 / 2}-w^{4} \mathrm{D}_{31 / 2}^{\circ} \\
b^{2} \mathrm{H}_{51 / 2}-x^{4} \mathrm{G}_{51 / 2}^{\circ}\end{array}$ & $\begin{array}{l}\left(0.00 w_{3} D\right) 0.88,1.05,1.19,1.35,1.51,1.67,1.82 \\
(0.00) 1.54 \dagger \\
(0.00) 1.56 \dagger\end{array}$ \\
\hline $\begin{array}{l}2840.43 \\
2840.01 \\
2839.23 \\
2838.78 \\
2837.96\end{array}$ & $\begin{array}{r}12 \\
85 \\
12 \\
65 \\
4\end{array}$ & $\begin{array}{l}35195.61 \\
35200.82 \\
35210.49 \\
35216.07 \\
35226.24\end{array}$ & $\begin{array}{l}a^{2} \mathrm{D}_{11 / 2}-y{ }^{4} \mathrm{P}_{21 / 2}^{\circ} \\
a^{4} \mathrm{H}_{41 / 2}-z^{4} \mathrm{I}_{51 / 2}^{\circ} \\
c^{4} \mathrm{D}_{31 / 2}-w^{4} \mathrm{D}_{31 / 2}^{\circ} \\
a^{4} \mathrm{H}_{61 / 2}-z^{4} \mathrm{I}_{61 / 2}^{\circ}\end{array}$ & $\begin{array}{l}(0.00) 0.96 \\
(0.00) 1.06 \dagger \\
(0.00) 1.44\end{array}$ \\
\hline $\begin{array}{l}\text { 2837. } 88 \\
2836.47 \\
2835.63 \\
2834.28 \\
2834.24\end{array}$ & $\begin{array}{r}20 \\
30 \\
200 \\
35 \\
60\end{array}$ & $\begin{array}{l}\text { 35227. } 24 \\
\text { 35244. } 75 \\
\text { 35255. } 19 \\
\text { 35271. } 98 \\
\text { 35272. } 48\end{array}$ & $\begin{array}{l}a{ }^{4} \mathrm{H}_{31 / 2}-z^{4} \mathrm{G}_{41 / 2}^{\circ} \\
b^{2} \mathrm{~F}_{31 / 2}-y^{2} \mathrm{~F}_{31 / 2}^{\circ} \\
a^{6} \mathrm{D}_{41 / 2}-z^{6} \mathrm{~F}_{51 / 2}^{\circ} \\
d^{2} \mathrm{~F}_{31 / 2}-v^{2} \mathrm{G}_{41 / 2}^{\circ} \\
a^{2} \mathrm{H}_{41 / 2}-y^{2} \mathrm{G}_{31 / 2}^{\circ}\end{array}$ & $\begin{array}{l}(\mathbf{0 . 2 4}, 0.68,1.14,1.61) 0.90,1.35,1.83,2.26, \mathbf{2 . 6 7} \\
(0.00) 1.16 \\
\left(0.00 w_{3} D\right) 1.03 A \\
(0.00) 1.13 \dagger \\
(0.00) 0.94 \dagger\end{array}$ \\
\hline $\begin{array}{l}\text { 2833. } 37 \\
2832.45 \\
2830.60 \\
2830.46 \\
2830.24\end{array}$ & $\begin{array}{r}8 \\
60 \\
60 \\
100 \\
10\end{array}$ & $\begin{array}{l}\text { 35283. } 31 \\
\text { 35294. } 76 \\
\text { 35317. } 83 \\
\text { 35319. } 58 \\
\text { 35322. } 32\end{array}$ & $\begin{array}{c}b^{2} \mathrm{~F}_{21 / 2}-y^{2} \mathrm{~F}_{31 / 2} \\
a^{2} \mathrm{H}_{51 / 2}-y^{2} \mathrm{G}_{41 / 2}^{\circ} \\
a^{4} \mathrm{H}_{61 / 2}-z^{4} \mathrm{G}_{51 / 2}^{\circ} \\
a^{4} \mathrm{H}_{51 / 2}-z^{4} \mathrm{I}_{61 / 2}^{\circ} \\
b^{4} \mathrm{G}_{31 / 2}-y^{4} \mathrm{H}_{31 / 2}^{\circ}\end{array}$ & $\begin{array}{l}(0.00 w) 1.64 B \dagger \\
(0.00) 1.03 \\
\left(0.00 w_{1} D\right) 1.06 A \\
\left(0.00 w_{1} D\right) 1.06 A\end{array}$ \\
\hline $\begin{array}{l}\text { 2830. } 08 \\
2828.79 \\
2827.95 \\
2826.42 \\
2826.15\end{array}$ & $\begin{array}{r}8 \\
15 \\
15 \\
7 \\
10\end{array}$ & $\begin{array}{l}35324.32 \\
35340.43 \\
35350.93 \\
35370.06 \\
35373.44\end{array}$ & $\begin{array}{l}a^{4} \mathrm{H}_{41 / 2}-z^{2} \mathrm{C}_{31 / 2}^{\circ} \\
a^{2} \mathrm{D}_{11 / 2}-z^{2} \mathrm{P}_{01 / 2}^{\circ}\end{array}$ & $\begin{array}{l}(0.00) 0.84 \dagger \\
(0.00) 0.76 \\
(0.00) 1.24 \dagger \\
(0.41) 1.52 \dagger \\
(0.72) 1.09 \dagger\end{array}$ \\
\hline
\end{tabular}


TABLE 1. Wavelengths of Cr II in air-Continued

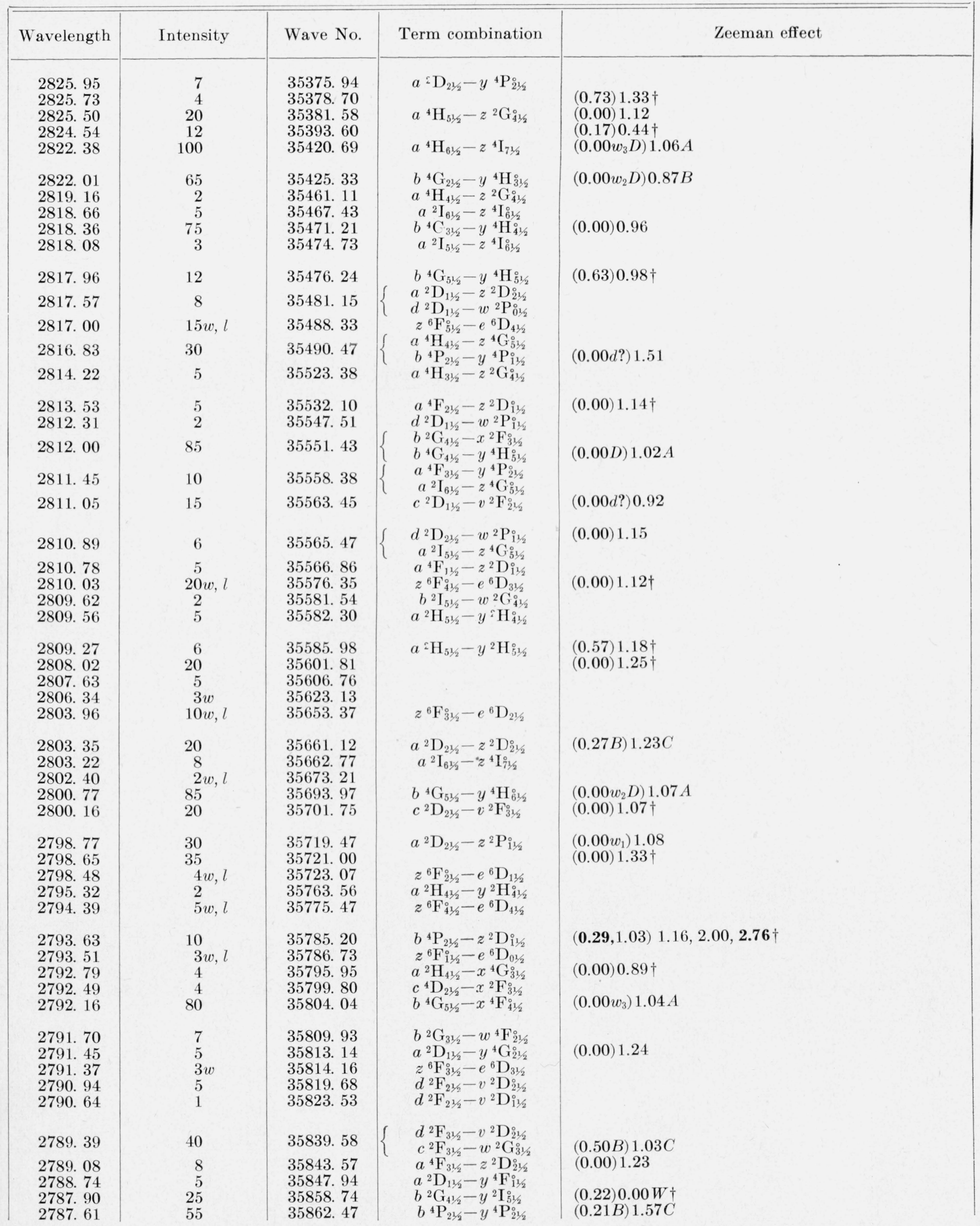


TABLE 1. Wavelengths of $\mathrm{Cr}$ II in air-Continued

\begin{tabular}{|c|c|c|c|c|}
\hline Wavelength & Intensity & Wave No. & Term combination & Zeeman effect \\
\hline $\begin{array}{l}2787.30 \\
2787.13 \\
2786.46 \\
2786.30 \\
2785.69\end{array}$ & $\begin{array}{l}5 \\
2 w \\
10 \\
2 \\
65\end{array}$ & $\begin{array}{l}35866.46 \\
35868.64 \\
35877.27 \\
35879.33 \\
35887.18\end{array}$ & $\begin{array}{l}a^{2} \mathrm{H}_{51 / 2}-x^{4} \mathrm{G}_{41 / 2}^{\circ} \\
z^{6} \mathrm{~F}_{01 / 2}^{\circ}-e^{6} \mathrm{D}_{01 / 2}^{\circ} \\
c^{4} \mathrm{D}_{01 / 2}-w^{4} \mathrm{~F}_{11 / 2}^{\circ} \\
b^{4} \mathrm{G}_{41 / 2}-x^{4} \mathrm{~F}_{41 / 2}^{\circ} \\
b^{4} \mathrm{G}_{41 / 2}-x^{4} \mathrm{~F}_{31 / 2}^{0}\end{array}$ & $\begin{array}{l}(0.20) 0.20, \mathbf{0 . 6 1} \\
(0.00) 1.17\end{array}$ \\
\hline $\begin{array}{l}2785.32 \\
2785.10 \\
2784.30 \\
2783.84 \\
2782.59\end{array}$ & $\begin{array}{l}2 \\
10 \\
4 w \\
20 \\
25\end{array}$ & $\begin{array}{l}35891.95 \\
35894.79 \\
35905.10 \\
35911.03 \\
35927.16\end{array}$ & $\begin{array}{l}c^{2} \mathrm{G}_{41 / 2}-w^{2} \mathrm{G}_{31 / 2}^{\circ} \\
a^{4} \mathrm{~F}_{21 / 2}-z^{2} \mathrm{D}_{21 / 2}^{\circ} \\
c^{4} \mathrm{D}_{11 / 2}-w^{4} \mathrm{~F}_{11 / 2}^{\circ} \\
b^{2} \mathrm{G}_{31 / 2}-x^{2} \mathrm{~F}_{21 / 2}^{21}\end{array}$ & $\begin{array}{l}(0.50 B) 1.09 C \dagger \\
(0.00) 0.45 \\
(0.50,1.20) 0.00,0.78,1.60 \dagger \\
(0.00) 0.86\end{array}$ \\
\hline $\begin{array}{l}2782.44 \\
2782.36 \\
2782.13 \\
2781.55 \\
2781.07\end{array}$ & $\begin{array}{l}3 \\
40 \\
4 \\
4 w, l \\
25\end{array}$ & $\begin{array}{l}35929.10 \\
35930.13 \\
35933.10 \\
35940.60 \\
35946.80\end{array}$ & $\begin{array}{c}a^{4} \mathrm{~F}_{11 / 2}-z^{2} \mathrm{D}_{21 / 2}^{\circ} \\
b^{4} \mathrm{G}_{21 / 2}-x^{4} \mathrm{~F}_{11 / 2}^{\circ} \\
c^{2} \mathrm{~F}_{31 / 2}-w^{2} \mathrm{G}_{41 / 2}^{\circ} \\
z^{4} \mathrm{D}_{31 / 2}^{\circ}-e^{4} \mathrm{~F}_{31 / 2}^{\circ} \\
b^{2} \mathrm{G}_{31 / 2}-x^{2} \mathrm{H}_{41 / 2}^{\circ}\end{array}$ & $\begin{array}{l}(0.00) 0.57 \\
(0.00) 0.94 \dagger\end{array}$ \\
\hline 2780.89 & 25 & 35949.12 & $b{ }^{4} \mathrm{P}_{11 / 2}-y{ }^{4} \mathrm{P}_{01 / 2}^{\circ}$ & $(0.43) 1.34,2.22 \dagger$ \\
\hline 2780.30 & 85 & 35956.75 & $\begin{array}{l}c^{4} \mathrm{D}_{11 / 2}-w^{4} \mathrm{~F}_{21 / 2}^{0} \\
b^{4} \mathrm{G}_{31 / 2}-x^{4} \mathrm{~F}_{21 / 2}^{\circ}\end{array}$ & $(0.00) 0.94 \dagger$ \\
\hline $\begin{array}{l}2778.94 \\
2778.51 \\
2778.27\end{array}$ & $\begin{array}{r}10 \\
5 \\
4\end{array}$ & $\begin{array}{l}35974.35 \\
35979.92 \\
\text { 35983. } 02\end{array}$ & $\begin{array}{l}c^{2} \mathrm{~F}_{21 / 2}-w^{2} \mathrm{G}_{31 / 2}^{\circ} \\
a^{2} \mathrm{~F}_{21 / 2}-z^{2} \mathrm{~F}_{21 / 2}^{\circ} \\
a^{2} \mathrm{D}_{21 / 2}-y^{4} \mathrm{G}_{31 / 2}^{\circ}\end{array}$ & $(0.00) 0.95$ \\
\hline $\begin{array}{l}2778.06 \\
2776.65 \\
2776.00 \\
2774.44 \\
2773.30\end{array}$ & $\begin{array}{l}70 \\
20 \\
3 w, l \\
50 \\
30\end{array}$ & $\begin{array}{l}35985.74 \\
36004.01 \\
36012.45 \\
36032.70 \\
36047.50\end{array}$ & $\begin{array}{l}c^{2} \mathrm{G}_{41 / 2}-w^{2} \mathrm{G}_{41 / 2}^{\circ} \\
c^{4} \mathrm{D}_{21 / 2}-w^{4} \mathrm{~F}_{21 / 2}^{\circ} \\
z^{4} \mathrm{D}_{11 / 2}-e^{4} \mathrm{~F}_{11 / 2} \\
c^{2} \mathrm{G}_{31 / 2}-w^{2} \mathrm{G}_{31 / 2}^{\circ} \\
b^{4} \mathrm{P}_{11 / 2}-y^{4} \mathrm{P}_{11 / 2}^{\circ}\end{array}$ & $\begin{array}{l}(0.00) 1.11 \\
(0.85) 1.19 \dagger \\
(0.34 B) 0.96 C \\
(0.07) 1.71\end{array}$ \\
\hline $\begin{array}{l}2772.33 \\
2771.89 \\
2771.27 \\
2769.92 \\
2769.70\end{array}$ & $\begin{array}{l}8 \\
20 w, l \\
12 \\
10 w, l \\
3 w, l\end{array}$ & $\begin{array}{l}36060.12 \\
36065.84 \\
36073.90 \\
36091.49 \\
36094.36\end{array}$ & $\begin{array}{l}b^{4} \mathrm{G}_{211 / 2}-x{ }^{4} \mathrm{~F}_{21 / 2}^{\circ} \\
z^{4} \mathrm{D}_{31 / 2}^{\circ}-e^{4} \mathrm{~F}_{41 / 2}^{\circ} \\
c^{4} \mathrm{D}_{111 / 2}-x^{2} \mathrm{~F}_{21 / 2}^{\circ} \\
z^{4} \mathrm{D}_{11 / 2}^{\circ}-e e^{4} \mathrm{~F}_{21 / 2} \\
z^{4} \mathrm{D}_{01 / 2}^{\circ}-e{ }^{4} \mathrm{~F}_{11 / 2}^{\circ}\end{array}$ & $\begin{array}{l}(1.00) \\
(0.00 D) 1.15 C \\
0.00 w) 0.56 A \dagger \\
(0.00 w) 0.96 A \dagger\end{array}$ \\
\hline $\begin{array}{l}2769.29 \\
2768.59 \\
2768.16 \\
2767.92 \\
2767.62\end{array}$ & $\begin{array}{l}8 w, l \\
50 \\
10 \\
3 \\
20\end{array}$ & $\begin{array}{l}36099.70 \\
36108.82 \\
36114.43 \\
36117.56 \\
36121.48\end{array}$ & $\begin{array}{l}z^{4} \mathrm{D}_{21 / 2}^{\circ}-e^{4} \mathrm{~F}_{31 / 2} \\
c^{4} \mathrm{D}_{21 / 2}-w^{4} \mathrm{~F}_{31 / 2}^{\circ} \\
a^{4} \mathrm{~F}_{41 / 2}-y^{4} \mathrm{G}_{31 / 2}^{\circ} \\
c^{4} \mathrm{D}_{11 / 2}-x{ }^{4} \mathrm{P}_{21 / 2}^{\circ}\end{array}$ & $\begin{array}{l}(0.00) 1.18 \dagger \\
\left(0.00 w_{3} D\right) 0.93 A \\
(0.00 D) 2.01 B \dagger\end{array}$ \\
\hline $\begin{array}{l}\text { 2767. } 26 \\
2766.55 \\
2765.86 \\
2765.62 \\
2765.46\end{array}$ & $\begin{array}{r}10 \\
150 \\
20 \\
12 \\
20\end{array}$ & $\begin{array}{l}\text { 36126. } 18 \\
36135.45 \\
36144.46 \\
36147.60 \\
36149.69\end{array}$ & $\begin{array}{l}c{ }^{2} \mathrm{G}_{31 / 2}-w^{2} \mathrm{G}_{41 / 2}^{\circ} \\
a^{6} \mathrm{D}_{41 / 2}^{\circ}-z^{6} \mathrm{P}_{31 / 2}^{\circ} \\
b^{2} \mathrm{G}_{41 / 2}-x^{2} \mathrm{H}_{51 / 2}^{\circ} \\
b^{4} \mathrm{P}_{21 / 2}-z^{2} \mathrm{D}_{21 / 2}^{\circ} \\
a^{4} \mathrm{~F}_{41 / 2}-y^{4} \mathrm{G}_{51 / 2}^{\circ}\end{array}$ & $\begin{array}{l}(0.00) 1.49 \dagger \\
(\mathbf{0 . 0 8}, 0.24,0.38,0.55) \mathbf{1 . 0 0}, 1.15,1.31,1.48,1.63 \\
(0.00) 0.87 \dagger \\
(0.74) \cdot{ }^{0.00)} 1.11 \dagger \\
(0.7\end{array}$ \\
\hline $\begin{array}{l}2765.13 \\
2764.96 \\
2764.28 \\
2763.97 \\
2763.59\end{array}$ & $\begin{array}{r}4 \\
10 \\
15 \\
12 \\
20\end{array}$ & $\begin{array}{l}36154.01 \\
36156.23 \\
36165.12 \\
36169.18 \\
36174.15\end{array}$ & $\begin{array}{l}c^{4} \mathrm{D}_{31 / 2}-w^{4} \mathrm{~F}_{31 / 2} \\
a^{2} \mathrm{~F}_{21 / 2}-z^{2} \mathrm{~F}_{31 / 2}^{\circ} \\
a^{4} \mathrm{~F}_{31 / 2}-y^{4} \mathrm{G}_{31 / 2}^{\circ} \\
c^{4} \mathrm{D}_{21 / 2}-x^{4} \mathrm{P}_{21 / 2}^{\circ} \\
a^{4} \mathrm{~F}_{41 / 2}-y^{4} \mathrm{~F}_{31 / 2}^{\circ}\end{array}$ & $\begin{array}{l}(0.60 B) 1.11 C^{\dagger} \\
(0.44 B) 1.40 C^{\dagger} \\
(0.00) 1.67 \dagger\end{array}$ \\
\hline $\begin{array}{l}2762.78 \\
2762.58 \\
2761.16 \\
2760.83 \\
2760.53\end{array}$ & $\begin{array}{r}10 \\
140 \\
5 \\
15 \\
25\end{array}$ & $\begin{array}{l}36184.76 \\
36187.38 \\
36205.99 \\
36210.31 \\
36214.25\end{array}$ & $\begin{array}{c}a^{4} \mathrm{~F}_{31 / 2}-y{ }^{4} \mathrm{G}_{41 / 2}^{\circ} \\
a{ }^{6} \mathrm{D}_{31 / 2}-z^{6} \mathrm{P}_{21 / 2}^{0} \\
b{ }^{4} \mathrm{P}_{21 / 2}-z^{2} \mathrm{P}_{11 / 2}^{\circ} \\
c^{4} \mathrm{D}_{31 / 2}-x{ }^{4} \mathrm{P}_{21 / 2}^{\circ}\end{array}$ & $\begin{array}{l}(\mathbf{0 . 1 7}, 0.46,0.75) \mathbf{0 . 8 2}, 1.15,1.45,1.74,2.04,2.34 \\
(0.00) 0.86 \dagger \\
(0.00) 1.22 \dagger\end{array}$ \\
\hline $\begin{array}{l}2760.36 \\
2760.20 \\
2760.04 \\
2759.73 \\
2759.40\end{array}$ & $\begin{array}{l}20 \\
12 \\
20 \\
30 \\
50\end{array}$ & $\begin{array}{l}36216.47 \\
36218.58 \\
36220.67 \\
36224.74 \\
36229.08\end{array}$ & $\begin{array}{l}a^{4} \mathrm{~F}_{21 / 2}-y^{4} \mathrm{G}_{31 / 2}^{\circ} \\
a^{4} \mathrm{~F}_{31 / 2}-y^{4} \mathrm{~F}_{21 / 2}^{\circ} \\
b^{4} \mathrm{G}_{21 / 2}-y^{2} \mathrm{D}_{11 / 2}^{\circ} \\
a^{4} \mathrm{~F}_{31 / 2}-y^{4} \mathbf{F}_{31 / 2}^{\circ} \\
a^{4} \mathrm{~F}_{41 / 2}-y^{4} \mathbf{F}_{41 / 2}^{\circ}\end{array}$ & $\begin{array}{l}(0.00) 1.11 \dagger \\
(0.00) 0.67 \\
(0.22) 1.05 \\
(0.00) 1.32\end{array}$ \\
\hline $\begin{array}{l}2759.23 \\
2758.99 \\
2758.61 \\
2757.72 \\
2756.96\end{array}$ & $\begin{array}{r}7 \\
40 \\
15 \\
80 \\
20\end{array}$ & $\begin{array}{l}36231.31 \\
36234.46 \\
36239.45 \\
36251.14 \\
36261.14\end{array}$ & $\begin{array}{l}c^{4} \mathrm{D}_{31 / 2}-w^{4} \mathrm{~F}_{41 / 2}^{\circ} \\
a^{2} \mathrm{~F}_{21 / 2}-y^{4} \mathrm{H}_{31 / 2}^{1} \\
a^{6} \mathrm{D}_{21 / 2}-z^{6} \mathrm{P}_{11 / 2}^{\circ} \\
a^{4} \mathrm{~F}_{11 / 2}-y^{4} \mathrm{G}_{21 / 2}^{11 / 2}\end{array}$ & $\begin{array}{l}(0.00 D) 1.01 A \\
(0.00) 1.10 \dagger \\
(\mathbf{0 . 3 6}, 1.09) \mathbf{0 . 6 0}, 1.33,2.04,2.76 \\
(0.00) 0.79 \dagger\end{array}$ \\
\hline
\end{tabular}


TABLE 1. Wavelengths of $\mathrm{Cr}$ II in air-Continued

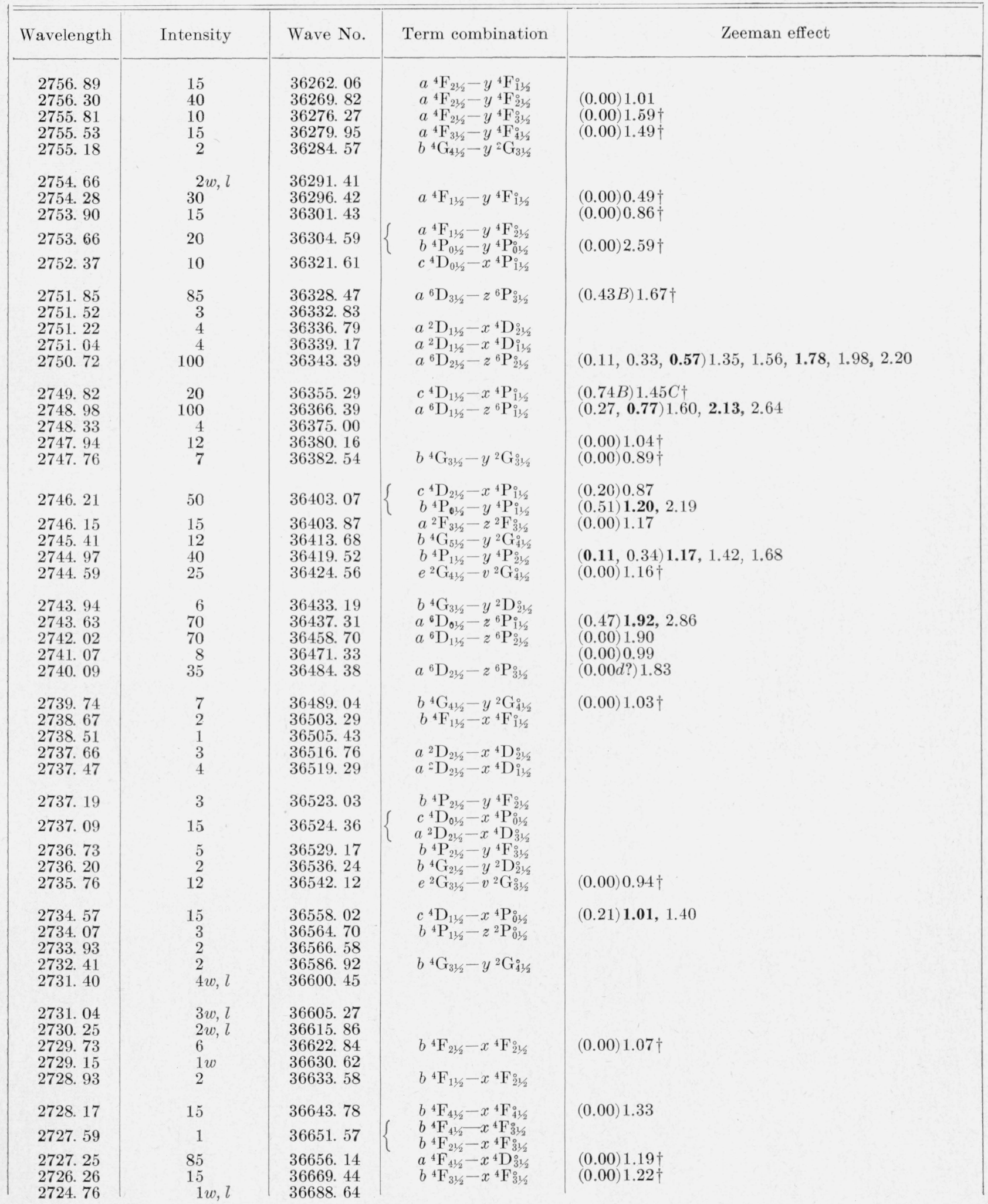


TABLE 1. Wavelengths of $\mathrm{Cr}$ II in air-Continued

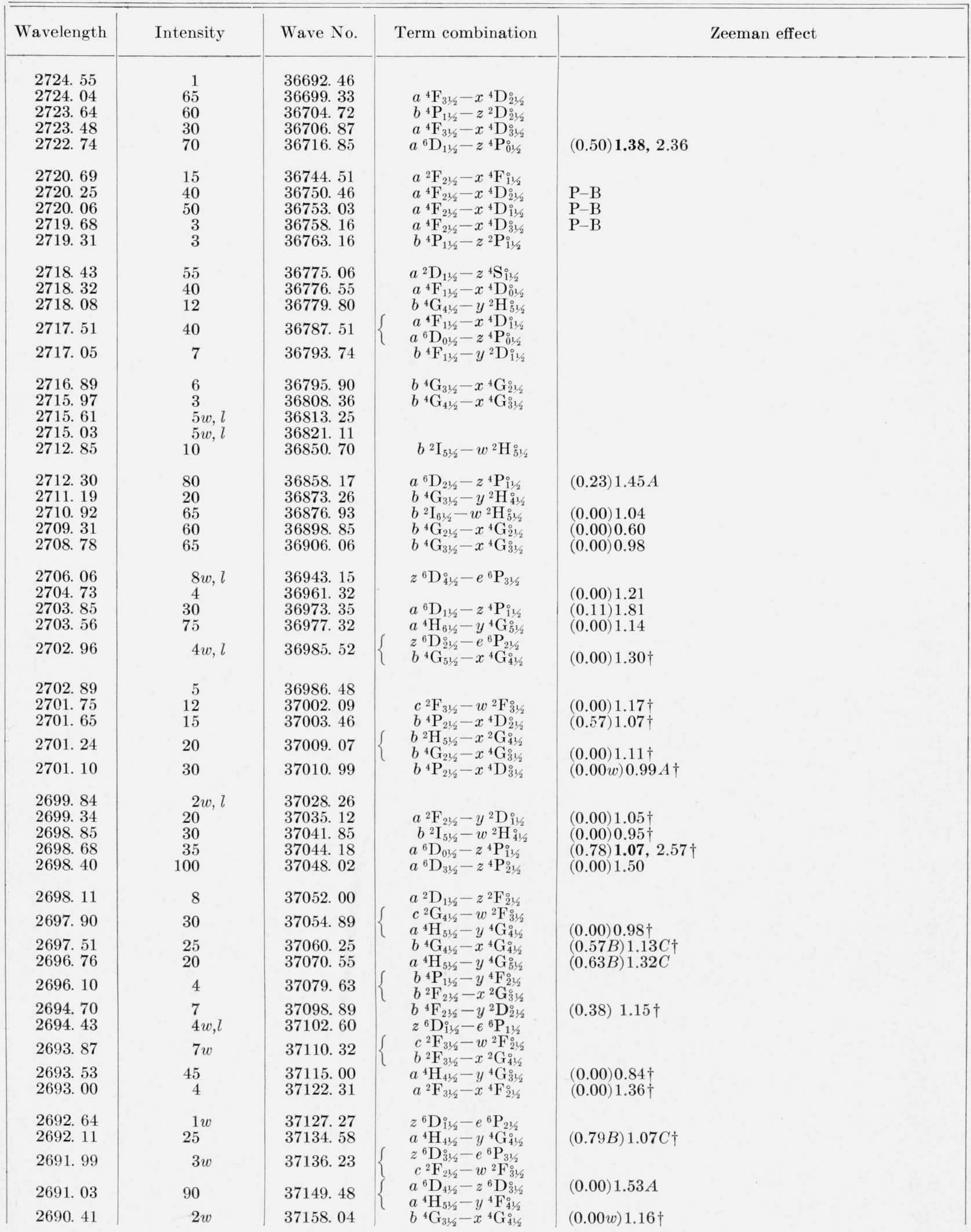


TABLE 1. Wavelengths of $\mathrm{Cr}$ II in air-Continued

\begin{tabular}{|c|c|c|c|c|}
\hline Wavelength & Intensity & Wave No. & Term combination & Zeeman effect \\
\hline $\begin{array}{l}2690.34 \\
2689.79 \\
2689.20 \\
2689.03 \\
2688.41\end{array}$ & $\begin{array}{l}8 w \\
10 \\
35 \\
20 \\
45\end{array}$ & $\begin{array}{l}37159.01 \\
37166.61 \\
37174.76 \\
37177.11 \\
37185.68\end{array}$ & $\begin{array}{c}b{ }^{4} \mathrm{G}_{41 / 2}^{\circ}-y^{2} \mathrm{~F}_{21 / 2}^{\circ} a^{4} \mathrm{H}_{41 / 2}-y^{4} \mathrm{~F}_{31 / 2}^{\circ} \\
a^{4} \mathrm{H}_{31 / 2}-y{ }^{4} \mathrm{G}_{31 / 2}^{\circ} \\
b^{4} \mathrm{G}_{51 / 2}-x{ }^{4} \mathrm{G}_{51 / 2}^{\circ}\end{array}$ & $\begin{array}{l}(0.00 w) 0.46 A \dagger \\
(0.50 B) 1.20 C \dagger\end{array}$ \\
\hline $\begin{array}{l}\text { 2688. } 28 \\
\text { 2688. } 14 \\
2687.60 \\
2687.09 \\
2686.66\end{array}$ & $\begin{array}{r}55 \\
5 \\
3 \\
65 \\
4\end{array}$ & $\begin{array}{l}37187.48 \\
37189.42 \\
37196.89 \\
37203.95 \\
37209.90\end{array}$ & $\begin{array}{c}a^{4} \mathrm{H}_{31 / 2}-y^{4} \mathrm{G}_{21 / 2}^{\circ} \\
c^{2} \mathrm{D}_{21 / 2}-w^{2} \mathrm{P}_{11 / 2}^{\circ} \\
a^{4} \mathrm{H}_{31 / 2}-y^{4} \mathrm{G}_{41 / 2}^{\circ} \\
a^{6} \mathrm{D}_{21 / 2}-z^{4} \mathrm{P}_{21 / 2}^{0} \\
a^{2} \mathrm{I}_{51 / 2}-y^{4} \mathrm{G}_{41 / 2}^{\circ}\end{array}$ & $\begin{array}{l}(0.00) 0.75 \dagger \\
(0.00) 1.64\end{array}$ \\
\hline $\begin{array}{l}2686.40 \\
2686.00 \\
2685.66\end{array}$ & $\begin{array}{l}6 \\
8 \\
2 w, l\end{array}$ & $\begin{array}{l}37213.50 \\
37219.05 \\
37223.76\end{array}$ & $\begin{array}{c}a^{2} \mathrm{G}_{41 / 2}-w^{4} \mathrm{D}_{31 / 2}^{\circ} \\
a^{2} \mathrm{I}_{61 / 2}-y{ }^{4} \mathrm{G}_{51 / 2}^{\circ}\end{array}$ & \\
\hline 2685. 19 & 18 & 37230. 27 & $\left\{\begin{array}{l}a^{4} \mathrm{H}_{41 / 2}-y^{4} \mathrm{~F}_{41 / 2}^{0} \\
a^{4} \mathrm{H}_{31 / 2}-y^{4} \mathrm{~F}_{21 / 2}^{0}\end{array}\right.$ & $(0.00 w) 0.00 w \dagger$ \\
\hline 2685. 04 & 18 & 37232.35 & $a^{2} \mathrm{D}_{21 / 2}-z^{2} \mathrm{~F}_{21 / 2}^{01 / 2}$ & $(0.57) 0.94$ \\
\hline $\begin{array}{l}\text { 2684. } 72 \\
\text { 2684. } 09 \\
2683.73 \\
\text { 2683. } 45 \\
\text { 2682. } 95\end{array}$ & $\begin{array}{r}7 \\
8 \\
4 \\
20 \\
1\end{array}$ & $\begin{array}{l}37236.79 \\
37245.53 \\
37250.53 \\
37254.41 \\
37261.35\end{array}$ & $\begin{array}{l}a^{4} \mathrm{H}_{31 / 2}-y^{4} \mathrm{~F}_{31 / 2}^{o} \\
c^{2} \mathrm{~F}_{21 / 2}-w^{2} \mathbf{F}_{21 / 2}^{\circ} \\
c^{2} \mathrm{D}_{11 / 2}-w^{2} \mathrm{P}_{11 / 2}^{\circ} \\
c^{2} \mathrm{G}_{41 / 2}-w^{2} \mathrm{H}_{51 / 2}^{\circ} \\
b^{4} \mathrm{G}_{41 / 2}-x^{4} \mathrm{G}_{51 / 2}^{\circ}\end{array}$ & $\begin{array}{l}(0.00) 0.82 \\
(0.00) 0.82 \dagger \\
(0.00) 1.06\end{array}$ \\
\hline $\begin{array}{l}2682.50 \\
2682.25 \\
2681.07 \\
2680.85 \\
2680.32\end{array}$ & $\begin{array}{l}2 w, l \\
2 w, l \\
3 \\
5 \\
15\end{array}$ & $\begin{array}{l}37267.60 \\
37271.08 \\
37287.48 \\
37290.54 \\
37297.91\end{array}$ & $\begin{array}{c}a^{4} \mathrm{H}_{41 / 2}-z^{2} \mathrm{I}_{51 / 2}^{\circ} \\
a^{4} \mathrm{H}_{51 / 2}-z^{2} \mathrm{I}_{61 / 2}^{\circ} \\
a^{2} \mathrm{~S}_{01 / 2}-x^{2} \mathrm{P}_{11 / 2}^{\circ}\end{array}$ & $\begin{array}{l}(0.00) 0.79 \dagger \\
(0.00) 0.92 \dagger \\
(0.33) \mathbf{0 . 9 2}, 1.70 \dagger\end{array}$ \\
\hline $\begin{array}{l}2680.16 \\
2679.89 \\
2678.79 \\
2677.19 \\
2677.13\end{array}$ & $\begin{array}{r}8 \\
15 \\
100 \\
125 \\
100\end{array}$ & $\begin{array}{l}37300.14 \\
37303.90 \\
37319.22 \\
37341.52 \\
37342.36\end{array}$ & $\begin{array}{l}a^{2} \mathrm{~F}_{21 / 2}-y^{2} \mathrm{G}_{31 / 2}^{\circ} \\
c^{2} \mathrm{G}_{31 / 2}-w^{2} \mathrm{~F}_{21 / 2}^{\circ} \\
a^{6} \mathrm{D}_{11 / 2}-z^{4} \mathrm{P}_{21 / 2}^{\circ} \\
a^{6} \mathrm{D}_{41 / 2}-z^{6} \mathrm{D}_{41 / 2}^{\circ} \\
a^{6} \mathrm{D}_{31 / 2}-z^{6} \mathrm{D}_{31 / 2}^{\circ}\end{array}$ & $\begin{array}{l}(0.00) 0.82 \dagger \\
(0.00 w) 1.25 \dagger \\
(\mathbf{0 . 1 5}, 0.34) \mathbf{1 . 2 8}, 1.48,1.76,1.97 \\
(0.00) 1.58 \\
(0.00) 1.58\end{array}$ \\
\hline $\begin{array}{l}\text { 2676. } 53 \\
2675.74 \\
\text { 2675. } 67 \\
2675.25 \\
2674.26\end{array}$ & $\begin{array}{l}5 \\
15 \\
20 \\
6 \\
7 w\end{array}$ & $\begin{array}{l}37350.73 \\
37361.75 \\
37362.73 \\
37368.60 \\
37382.42\end{array}$ & $\begin{array}{l}a^{2} \mathrm{~F}_{21 / 2}-y^{2} \mathrm{D}_{21 / 2}^{\circ} \\
a^{2} \mathrm{~S}_{01 / 2}^{\circ}-x^{2} \mathrm{P}_{01 / 2}^{\circ} \\
a^{2} \mathrm{I}_{51 / 2}-z^{2} \mathrm{I}_{51 / 2}^{\circ} \\
z^{4} \mathrm{~F}_{41 / 2}^{\circ}-e^{4} \mathrm{G}_{41 / 2}\end{array}$ & $\begin{array}{l}(0.64) 0.96 \dagger \\
(0.00) 0.93 \\
(0.00) 1.22 \dagger\end{array}$ \\
\hline $\begin{array}{l}\text { 2674. } 07 \\
\text { 2673. } 97 \\
\text { 2673. } 49 \\
\text { 2672. } 83 \\
\text { 2672. } 37\end{array}$ & $\begin{array}{l}8 w \\
8 w \\
3 \\
90 \\
15\end{array}$ & $\begin{array}{l}37385.08 \\
37386.48 \\
37393.19 \\
37402.43 \\
37408.86\end{array}$ & $\begin{array}{l}z^{4} \mathrm{~F}_{31 / 2}^{\circ}-e^{4} \mathrm{G}_{31 / 2} \\
z^{4} \mathrm{~F}_{21 / 2}^{2}-e^{4} \mathrm{G}_{21 / 2} \\
c^{2} \mathrm{~F}_{31 / 2}-w^{2} \mathrm{H}_{41 / 2}^{\circ} \\
a^{6} \mathrm{D}_{31 / 2}-z^{6} \mathrm{D}_{21 / 2}^{\circ} \\
a^{2} \mathrm{D}_{21 / 2}-z^{2} \mathrm{~F}_{31 / 2}^{\circ}\end{array}$ & $\begin{array}{l}(0.00 d) 1.55 \\
(0.00) 1.17\end{array}$ \\
\hline $\begin{array}{l}2671.80 \\
2671.02 \\
2670.90 \\
2670.24 \\
2670.06\end{array}$ & $\begin{array}{l}80 \\
2 \\
3 w \\
25 \\
30\end{array}$ & $\begin{array}{l}37416.84 \\
37427.77 \\
37429.45 \\
37438.70 \\
37441.23\end{array}$ & $\begin{array}{l}a{ }^{6} \mathrm{D}_{21 / 2}-z{ }^{6} \mathrm{D}_{11 / 2}^{\circ} \\
b{ }^{4} \mathrm{P}_{01 / 2}-y^{4} \mathrm{~F}_{11 / 2}^{\circ} \\
a^{2} \mathrm{I}_{61 / 2}-z^{2} \mathrm{I}_{61 / 2}^{\circ} \\
b^{4} \mathrm{P}_{21 / 2}-z^{4} \mathrm{~S}_{11 / 2}^{\circ}\end{array}$ & $\begin{array}{l}(0.00,0.25) 1.41,1.66,1.83 \\
(0.00) 1.12 \\
(\mathbf{0 . 1 9}, 0.56) 1.03,1.41,1.78,2.15\end{array}$ \\
\hline $\begin{array}{l}2669.07 \\
2668.71\end{array}$ & $\begin{array}{l}3 w \\
70\end{array}$ & $\begin{array}{l}37455.12 \\
37460.16\end{array}$ & $a^{6} \mathrm{D}_{11 / 2}-z^{6} \mathrm{D}_{01 / 2}^{\circ}$ & $(0.63) 1.24,2.51$ \\
\hline 2667. 89 & $25 w, l$ & 37471.68 & $\begin{array}{l}z^{4} \mathrm{~F}_{11 / 2}^{1}-e^{4} \mathrm{G}_{21 / 2} \\
b^{4} \mathrm{~F}_{11 / 2}-x^{4} \mathrm{G}_{21 / 3}^{\circ}\end{array}$ & $(0.00 w) 0.77 w \dagger$ \\
\hline $\begin{array}{l}\text { 2667. } 21 \\
\text { 2666. } 02\end{array}$ & $\begin{array}{r}4 \\
80\end{array}$ & $\begin{array}{l}\text { 37481. } 23 \\
37497.96\end{array}$ & $a^{6} \mathrm{D}_{21 / 2}-z^{6} \mathrm{D}_{31 / 2}^{\circ}$ & $(0.13) 1.43,1.65$ \\
\hline $\begin{array}{l}\text { 2665. } 58 \\
\text { 2664. } 28 \\
2663.67 \\
\text { 2663. } 42 \\
\text { 2663. } 28\end{array}$ & $\begin{array}{l}30 w, l \\
2 \\
45 \\
75 \\
30 w, l\end{array}$ & $\begin{array}{l}37504.15 \\
37522.45 \\
37531.04 \\
37534.56 \\
37536.53\end{array}$ & $\begin{array}{l}z^{4} \mathrm{~F}_{21 / 2}^{\circ}-e^{4} \mathrm{G}_{31 / 2} \\
b^{2} \mathrm{D}_{21 / 2}-w^{2} \mathrm{D}_{21 / 2}^{2} \\
a^{6} \mathrm{D}_{01 / 2}-z^{6} \mathrm{D}_{01 / 2}^{\circ} \\
a^{6} \mathrm{D}_{31 / 2}-z^{6} \mathrm{D}_{41 / 2}^{\circ} \\
z^{4} \mathrm{~F}_{31 / 2}^{\circ}-e^{4} \mathrm{G}_{41 / 2}^{\circ}\end{array}$ & $\begin{array}{l}(0.00) 1.02 \dagger \\
(0.00) 3.25 \\
(0.08) 1.51\end{array}$ \\
\hline $\begin{array}{l}\text { 2663. } 02 \\
2662.72 \\
2662.15 \\
2661.73 \\
2661.59\end{array}$ & $\begin{array}{r}10 \\
7 \\
4 \\
50 \\
10\end{array}$ & $\begin{array}{l}37540.20 \\
37544.43 \\
37552.47 \\
37558.39 \\
37560.37\end{array}$ & $\begin{array}{l}b^{4} \mathrm{~F}_{41 / 2}-y^{2} \mathrm{H}_{41 / 2}^{\circ} \\
b^{4} \mathrm{~F}_{41 / 2}-y^{2} \mathrm{H}_{51 / 2}^{\circ} \\
b^{4} \mathrm{P}_{11 / 2}-x^{4} \mathrm{D}_{01 / 2}^{\circ} \\
a^{6} \mathrm{D}_{21 / 2}-z^{6} \mathrm{D}_{21 / 2}^{\circ} \\
b^{4} \mathrm{P}_{11 / 2}^{\circ}-x^{4} \mathrm{D}_{21 / 2}^{\circ}\end{array}$ & $\begin{array}{l}(0.00 d ?) 1.04 \dagger \\
((0.09) 1.65\end{array}$ \\
\hline
\end{tabular}


TABLE 1. Wavelengths of $\mathrm{Cr}$ II in air-Continued

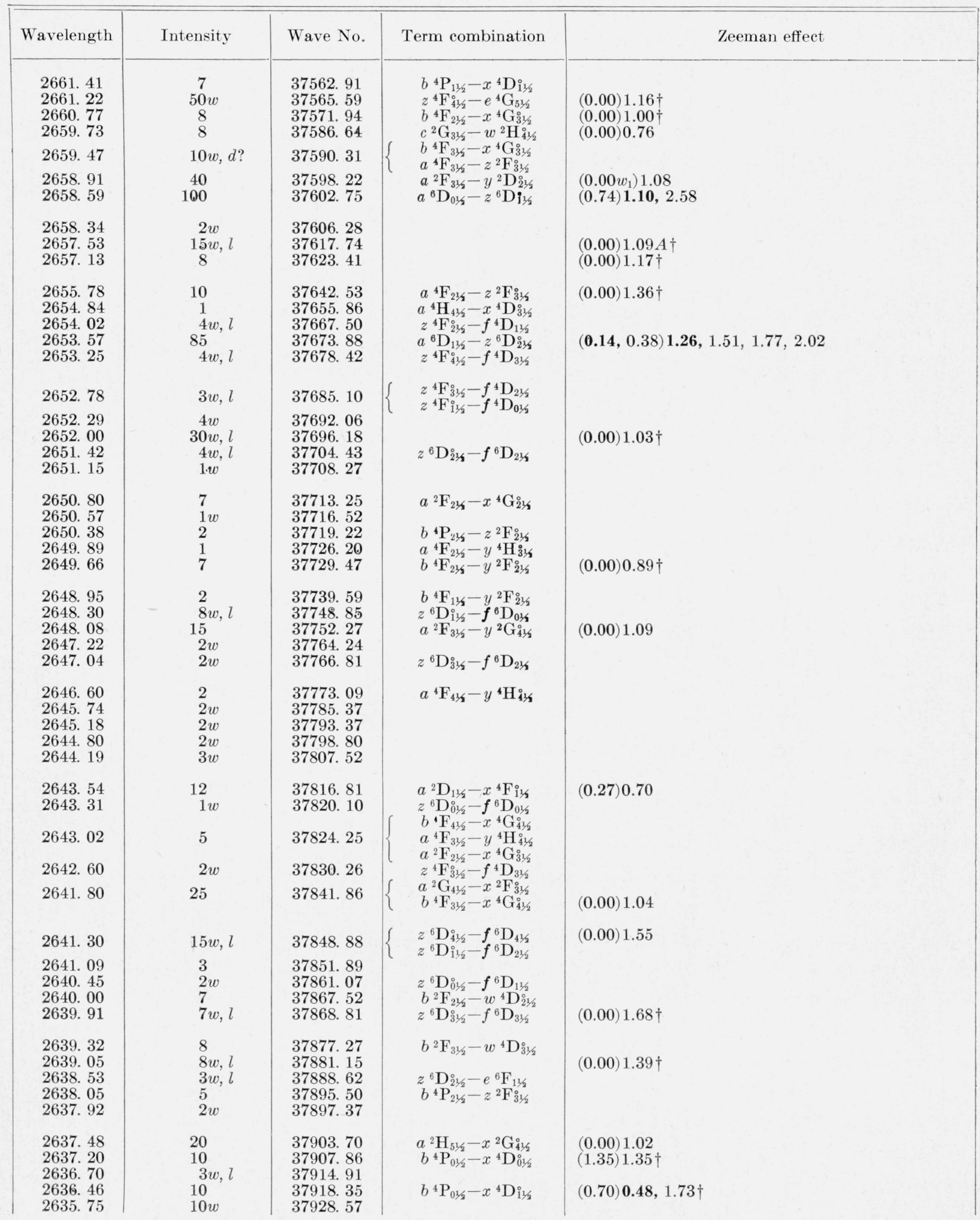


TABLE 1. Wavelengths of $\mathrm{Cr}$ II in air-Continued

\begin{tabular}{|c|c|c|c|c|}
\hline Wavelength & Intensity & Wave No. & Term combination & Zeeman effect \\
\hline $\begin{array}{l}2634.84 \\
2634.27 \\
2633.59 \\
2632.77 \\
2632.54\end{array}$ & $\begin{array}{c}2 w \\
12 w \\
10 w \\
5 \\
15 w, l\end{array}$ & $\begin{array}{l}37941.67 \\
37950.31 \\
37959.68 \\
37971.50 \\
37974.82\end{array}$ & $\begin{array}{l}z^{6} \mathrm{D}_{21 / 2}^{\circ}-e^{6} \mathrm{~F}_{21 / 2} \\
c^{2} \mathrm{~F}_{21 / 2}-x^{2} \mathrm{P}_{11 / 2}^{\circ} \\
y^{4} \mathrm{~F}_{41 / 2}^{\circ}-f^{4} \mathrm{G}_{51 / 2}^{\circ} \\
y^{4} \mathrm{~F}_{31 / 2}^{\circ}-f^{4} \mathrm{G}_{41 / 2}^{\circ}\end{array}$ & $(0.00) 1.28 \dagger$ \\
\hline $\begin{array}{l}2632.36 \\
2632.10 \\
2631.87 \\
2630.93 \\
2629.81\end{array}$ & $\begin{array}{l}20 w, l \\
3 \\
3 w \\
50 \\
2 w\end{array}$ & $\begin{array}{l}37977.42 \\
37981.17 \\
37984.48 \\
37998.06 \\
38014.24\end{array}$ & $\begin{array}{l}z^{6} \mathrm{D}_{11 / 2}-e{ }^{6} \mathrm{~F}_{01 / 2} \\
a^{2} \mathrm{~F}_{21 / 2}-y^{2} \mathrm{~F}_{21 / 2}^{11} \\
b^{4} \mathrm{P}_{11 / 2}-z^{4} \mathrm{~S}_{11 / 2}^{\circ}\end{array}$ & $(0.35) 1.61,1.87,2.13$ \\
\hline $\begin{array}{l}2629.58 \\
2629.42 \\
2629.04 \\
2628.88 \\
2628.72\end{array}$ & $\begin{array}{l}8 \\
4 w, l \\
5 \\
2, \text { Fe II? } \\
2 w\end{array}$ & $\begin{array}{l}38017.56 \\
38019.88 \\
38025.37 \\
38027.68 \\
38030.00\end{array}$ & $\begin{array}{l}a^{2} \mathrm{H}_{41 / 2}-x^{2} \mathrm{G}_{31 / 2}^{\circ} \\
z^{6} \mathrm{D}^{\circ}{ }^{\circ 1 / 2}-e^{6} \mathrm{~F}_{21 / 2} \\
b^{4} \mathrm{~F}_{41 / 2}-x^{4} \mathrm{G}_{51 / 2}^{\circ} \\
z^{6} \mathrm{D}_{11 / 2}^{\circ}-e^{6} \mathrm{~F}_{11 / 2}\end{array}$ & $(0.00) 0.94 \dagger$ \\
\hline $\begin{array}{l}2627.95 \\
2627.17 \\
2626.78 \\
2626.69 \\
2626.30\end{array}$ & $\begin{array}{l}35 w, l \\
3 w \\
20 \\
15 w \\
2 w\end{array}$ & $\begin{array}{l}38041.15 \\
38052.43 \\
38058.08 \\
38059.39 \\
38065.04\end{array}$ & $\begin{array}{l}z^{6} \mathrm{D}_{31 / 2}^{\circ}-f{ }^{6} \mathrm{D}_{11 / 2} \\
z^{6} \mathrm{D}_{21 / 2}^{\circ}-e^{6} \mathrm{~F}_{31 / 2} \\
c^{2} \mathrm{~F}_{31 / 2}-x^{2} \mathrm{~J}_{21 / 2}^{\circ} \\
z^{6} \mathrm{P}_{31 / 2}^{0}-e^{6} \mathrm{P}_{21 / 2}\end{array}$ & $\begin{array}{l}\left(0.00 w_{1}\right) 1.37 A \\
(0.00 w) 0.80 w \dagger\end{array}$ \\
\hline $\begin{array}{l}\text { 2625. } 87 \\
2625.00 \\
2624.66\end{array}$ & $\begin{array}{l}2 \\
2 w, l \\
4 w, l\end{array}$ & $\begin{array}{l}\text { 38071. } 27 \\
\text { 38083. } 89 \\
38088.82\end{array}$ & $a^{2} \mathbf{F}_{31 / 2}-x{ }^{4} \mathrm{G}_{31 / 2}^{\circ}$ & \\
\hline $\begin{array}{l}2623.82 \\
2623.39\end{array}$ & $\begin{array}{l}10 \mathrm{w}, l \\
30\end{array}$ & $\begin{array}{l}38101.02 \\
38107.26\end{array}$ & $\left\{\begin{array}{l}z^{6} \mathrm{D}_{01 / 2}^{\circ}-e^{6} \mathrm{~F}_{11 / 2} \\
z^{6} \mathrm{D}_{11 / 2}^{11}-e^{6} \mathrm{~F}_{21 / 2} \\
a^{2} \mathrm{D}_{11 / 2}-y^{2} \mathrm{D}_{11 / 2}^{\circ}\end{array}\right.$ & $(0.00 d ?) 0.70 \dagger$ \\
\hline $\begin{array}{l}2623.20 \\
2623.00 \\
2622.64 \\
2622.03 \\
2621.80\end{array}$ & $\begin{array}{l}40 w, l \\
5 w, l \\
4 w, l \\
3 \\
4 w, l\end{array}$ & $\begin{array}{l}38110.02 \\
38112.93 \\
38118.16 \\
38127.02 \\
38130.37\end{array}$ & $\begin{array}{l}z^{6} \mathrm{D}_{41 / 2}^{\circ}-e^{6} \mathrm{~F}_{41 / 2} \\
z^{6} \mathrm{D}_{31 / 2}^{\circ}-e^{6} \mathrm{~F}_{31 / 2}^{\circ} \\
a^{2} \mathrm{D}_{21 / 2}-x^{4} \mathrm{~F}_{21 / 2}^{\circ}\end{array}$ & \\
\hline $\begin{array}{l}2621.18 \\
2620.86 \\
2620.48 \\
2620.10 \\
2619.59\end{array}$ & $\begin{array}{c}2 w, l \\
5 \\
50 w, l \\
1 w \\
75 w, l\end{array}$ & $\begin{array}{l}38139.39 \\
38144.05 \\
38149.58 \\
38155.11 \\
38162.53\end{array}$ & $\begin{array}{l}z^{6} \mathrm{P}_{31 / 2}^{\circ}-e{ }^{6} \mathrm{P}_{31 / 2} \\
a^{2} \mathrm{D}_{21 / 2}-x{ }^{4} \mathrm{~F}_{31 / 2}^{\circ} \\
z^{6} \mathrm{D}_{11 / 2}^{\circ}-e{ }^{6} \mathrm{~F}_{51 / 2}^{\circ}\end{array}$ & $\begin{array}{l}(0.00) 1.73 \\
\left(0.00 w_{3}\right) 1.09 \mathrm{~A}\end{array}$ \\
\hline $\begin{array}{l}2618.77 \\
2618.63 \\
2618.49 \\
2617.50 \\
2617.03\end{array}$ & $\begin{array}{c}12 w \\
15 w, l \\
7 \\
3 w \\
1 w\end{array}$ & $\begin{array}{l}38174.48 \\
38176.52 \\
38178.57 \\
38193.01 \\
38199.86\end{array}$ & $\begin{array}{l}z^{6} \mathrm{P}_{21 / 2}^{0}-e^{6} \mathrm{P}_{11 / 2} \\
a^{4} \mathrm{H}_{51 / 2}-z^{2} \mathrm{H}_{41 / 2}^{0} \\
c^{2} \mathrm{~F}_{21 / 2}-x^{2} \mathrm{D}_{21 / 2}^{\circ} \\
z^{6} \mathrm{P}_{21 / 2}^{\circ}-e^{6} \mathrm{P}_{21 / 2}\end{array}$ & $(0.00) 1.85 \dagger$ \\
\hline $\begin{array}{l}2616.18 \\
2615.85 \\
2614.90 \\
2614.57 \\
2613.82\end{array}$ & $\begin{array}{l}50 w, l \\
1 \\
10 \\
50 w, l \\
3,+\mathrm{FeII}\end{array}$ & $\begin{array}{l}38212.28 \\
38217.09 \\
38230.98 \\
38235.80 \\
38246.77\end{array}$ & $\begin{array}{l}a^{2} \mathrm{G}_{31 / 2}-w^{4} \mathrm{~F}_{21 / 2}^{\circ} \\
a^{4} \mathrm{~F}_{21 / 2}-x^{4} \mathrm{~F}_{11 / 2}^{\circ} \\
b^{2} \mathrm{D}_{11 / 2}-v^{2} \mathrm{~F}_{21 / 2}^{\circ}\end{array}$ & $\begin{array}{l}(0.00) 1.34 \dagger \\
(0.00) 1.48\end{array}$ \\
\hline $\begin{array}{l}2613.51 \\
2613.14 \\
2612.56 \\
2612.34 \\
2612.08\end{array}$ & $\begin{array}{l}12 \\
10 w, l \\
15 \\
7 w \\
8\end{array}$ & $\begin{array}{l}38251.31 \\
38256.73 \\
38265.22 \\
38268.44 \\
38272.26\end{array}$ & $\begin{array}{l}c^{2} \mathrm{G}_{31 / 2}-x{ }^{2} \mathrm{D}_{21 / 2}^{\circ} \\
a^{4} \mathrm{~F}_{11 / 2}-x{ }^{4} \mathrm{~F}_{11 / 2}^{\circ} \\
z^{6} \mathrm{P}_{11 / 2}^{11}-e^{6} \mathrm{P}_{11 / 2}\end{array}$ & $\begin{array}{l}(0.00) 1.61 \mathrm{w} \dagger \\
(0.00 d ?) 0.52 \dagger \\
(0.00) 2.41 \dagger\end{array}$ \\
\hline $\begin{array}{l}\text { 2611. } 62 \\
2611.04 \\
2610.81 \\
2610.70 \\
2610.04\end{array}$ & $\begin{array}{l}20 \\
30 \\
50 w, l \\
40 w, l \\
20 w, l\end{array}$ & $\begin{array}{l}\text { 38278. } 99 \\
38287.49 \\
38290.87 \\
38292.48 \\
38302.16\end{array}$ & $\left\{\begin{array}{c}a^{4} \mathrm{~F}_{41 / 2}-x^{4} \mathrm{~F}_{41 / 2}^{\circ} \\
a^{4} \mathrm{~F}_{41 / 2}-x^{4} \mathrm{~F}_{31 / 2}^{\circ} \\
a^{2} \mathrm{D}_{21 / 2}-y^{2} \mathrm{D}_{11 / 2}^{\circ} \\
z^{6} \mathrm{P}_{21 / 2}^{\circ}-e^{6} \mathrm{P}_{31 / 2}^{\circ} \\
z^{6} \mathrm{P}_{11 / 2}^{0}-e^{6} \mathrm{P}_{21 / 2}^{\circ} \\
z^{6} \mathrm{D}_{31 / 2}^{\circ}-e^{6} \mathrm{~F}_{41 / 2}^{\circ}\end{array}\right.$ & $\begin{array}{l}(0.00) 1.15 \\
(0.00) 1.77 \dagger \\
\left(0.00 w_{2}\right) 1.29 A \\
(0.00) 1.14 \dagger\end{array}$ \\
\hline
\end{tabular}


TABLE 1. Wavelengths of Cr II in air-Continued

\begin{tabular}{|c|c|c|c|c|}
\hline Wavelength & Intensity & Wave No. & Term combination & Zeeman effect \\
\hline $\begin{array}{l}2609.55 \\
2609.24 \\
2609.11 \\
2608.80 \\
2608.60\end{array}$ & $\begin{array}{l}3 \\
4 w, l \\
1 \\
8 \\
1\end{array}$ & $\begin{array}{l}38309.35 \\
38313.90 \\
38315.81 \\
38320.37 \\
38323.30\end{array}$ & $\begin{array}{c}a^{4} \mathrm{~F}_{31 / 2}-x^{4} \mathrm{~F}_{21 / 2}^{\circ} \\
b^{2} \mathrm{G}_{41 / 2}-w^{2} \mathrm{~F}_{31 / 2}^{\circ} \\
a^{4} \mathrm{H}_{31 / 2}-z^{2} \mathrm{H}_{41 / 2}^{\circ} \\
a^{2} \mathrm{~F}_{31 / 2}-x^{4} \mathrm{G}_{41 / 2}^{\circ}\end{array}$ & $(0.00) 1.41 \dagger$ \\
\hline $\begin{array}{l}2608.29 \\
2608.17 \\
2607.90 \\
2607.85 \\
2607.64\end{array}$ & $\begin{array}{l}3 w \\
20 \\
50 \\
10 \\
10\end{array}$ & $\begin{array}{l}38327.86 \\
38329.62 \\
38333.59 \\
38334.32 \\
38337.56\end{array}$ & $\begin{array}{c}a^{4} \mathbf{F}_{31 / 2}-x^{4} \mathbf{F}_{41 / 2}^{\circ} \\
a^{2} \mathrm{I}_{51 / 2}-z^{2} \mathbf{H}_{41 / 2}^{\circ} \\
a^{2} \mathrm{G}_{31 / 2}-x^{2} \mathbf{F}_{21 / 2}^{\circ} \\
a^{4} \mathbf{F}_{31 / 2}-x^{4} \mathbf{F}_{31 / 2}^{0}\end{array}$ & $\begin{array}{l}(0.00) 1.38 \dagger \\
(0.00) 1.00 \\
(0.00) 1.38 \dagger\end{array}$ \\
\hline $\begin{array}{l}2607.06 \\
2606.65\end{array}$ & $\begin{array}{l}12 \\
4 w, l\end{array}$ & $\begin{array}{l}38345.93 \\
38351.97\end{array}$ & $a{ }^{4} \mathrm{H}_{61 / 2}-z{ }^{2} \mathrm{H}_{51 / 2}^{\circ}$ & $(\mathbf{0 . 1 3}, 0.30) \ldots ?$ \\
\hline 2606.53 & 25 & 38353. 74 & $\begin{array}{l}a^{2} \mathrm{G}_{31 / 2}-x^{2} \mathrm{H}_{41 / 2}^{\circ} \\
b{ }^{4} \mathrm{P}_{01 / 2}-z^{4} \mathrm{~S}_{11 / 2}\end{array}$ & $(0.36) 1.65, \ldots ?$ \\
\hline $\begin{array}{l}2606.07 \\
2605.63\end{array}$ & $\begin{array}{l}12 \\
15\end{array}$ & $\begin{array}{l}38360.51 \\
38366.98\end{array}$ & $a^{4} \mathbf{F}_{21 / 2}-x^{4} \mathbf{F}_{21 / 2}^{\circ} \mathbf{F}_{21 / 2}^{2}-x^{2} \mathrm{D}_{11 / 2}^{\circ}$ & $\begin{array}{l}(0.00) 1.07 \dagger \\
(0.00) 0.86 \dagger\end{array}$ \\
\hline $\begin{array}{l}\text { 2604. } 16 \\
\text { 2603. } 73 \\
2603.25 \\
2603.00 \\
2602.04\end{array}$ & $\begin{array}{l}20 \\
10 \\
2 w \\
10 w \\
3 w\end{array}$ & $\begin{array}{l}38388.64 \\
38394.98 \\
38402.05 \\
38405.74 \\
38419.91\end{array}$ & $\begin{array}{l}a^{4} \mathrm{~F}_{21 / 2}-x^{4} \mathrm{~F}_{31 / 2}^{\circ} \\
a^{4} \mathrm{~F}_{11 / 2}-x^{4} \mathrm{~F}_{21 / 2}^{\circ}\end{array}$ & $\begin{array}{l}(0.00) 1.60 \dagger \\
(0.00) 1.92 \dagger\end{array}$ \\
\hline $\begin{array}{l}2601.85 \\
2601.58 \\
2601.30 \\
2601.04 \\
2600.73\end{array}$ & $\begin{array}{l}10 \\
6 \\
3 w \\
8 \\
5 w\end{array}$ & $\begin{array}{l}38422.72 \\
38426.71 \\
38430.84 \\
38434.68 \\
38439.27\end{array}$ & $\begin{array}{l}a^{2} \mathrm{D}_{11 / 2}-y^{2} \mathrm{D}_{21 / 2}^{\circ} \\
a^{4} \mathrm{H}_{31 / 2}^{\circ}-z^{2} \mathrm{~F}_{21 / 2}^{21} \\
a^{2} \mathrm{G}_{41 / 2}-x^{2} \mathrm{H}_{51 / 2}^{\circ} \\
a^{4} \mathrm{H}_{51 / 2}-z^{2} \mathrm{H}_{51 / 2}^{\circ}\end{array}$ & $\begin{array}{l}(0.00) 1.61 \dagger \\
(0.00) 0.40 \dagger \\
(0.00) 0.90 \dagger\end{array}$ \\
\hline $\begin{array}{l}2599.65 \\
2599.04 \\
2598.73 \\
2598.48 \\
2598.06\end{array}$ & $\begin{array}{l}1 w \\
2 w \\
2 w \\
3 w \\
3\end{array}$ & $\begin{array}{l}38455.23 \\
38464.26 \\
38468.85 \\
38472.55 \\
38478.76\end{array}$ & $b^{2} \mathrm{G}_{31 / 2}-w^{2} \mathrm{~F}_{21 / 2}^{0}$ & \\
\hline $\begin{array}{l}2597.44 \\
2596.87 \\
2596.17 \\
2596.03 \\
2595.55\end{array}$ & $\begin{array}{l}2 w, l \\
8 \\
40 \\
25 \\
25\end{array}$ & $\begin{array}{l}\text { 38487. } 95 \\
38496.40 \\
\text { 38506. } 77 \\
\text { 38508. } 85 \\
38515.97\end{array}$ & $\begin{array}{l}a^{2} \mathrm{~F}_{31 / 2}-y^{2} \mathrm{~F}_{31 / 2}^{\circ} \\
b^{2} \mathrm{~F}_{31 / 2}^{\circ}-x^{2} \mathbf{F}_{31 / 2}^{\circ} \\
b^{2} \mathrm{G}_{41 / 2}-w^{2} \mathrm{H}_{51 / 2}^{\circ}\end{array}$ & $\begin{array}{l}(0.00) 1.24 \\
(0.00) 1.17 \dagger \\
(0.00) 1.17 \\
(0.00) 1.03 \dagger\end{array}$ \\
\hline $\begin{array}{l}2595.34 \\
2594.80 \\
2594.51 \\
2594.32 \\
2594.10\end{array}$ & $\begin{array}{l}4 w, l \\
1 w \\
1 w \\
7 \\
4 w, l\end{array}$ & $\begin{array}{l}38519.09 \\
38527.10 \\
38531.41 \\
38534.23 \\
38537.50\end{array}$ & $\begin{array}{l}a^{4} \mathrm{H}_{41 / 2}-z^{2} \mathrm{H}_{51 / 2}^{\circ} \\
b^{2} \mathrm{D}_{21 / 2}-v^{2} \mathrm{~F}_{31 / 2}^{\circ}\end{array}$ & $(0.00) 1.10 \dagger$ \\
\hline $\begin{array}{l}2593.92 \\
2593.49 \\
2593.10 \\
2592.86 \\
2592.42\end{array}$ & $\begin{array}{l}3 \\
8 \\
1 \\
3 \\
3 w, l\end{array}$ & $\begin{array}{l}38540.17 \\
38546.56 \\
38552.36 \\
38555.93 \\
38562.47\end{array}$ & $\begin{array}{c}a{ }^{4} \mathrm{H}_{41 / 2}-z^{2} \mathrm{~F}_{31 / 2}^{\circ} \\
b{ }^{2} \mathrm{~S}_{01 / 2}-w^{2} \mathrm{P}_{01 / 2}^{\circ} \\
a^{2} \mathrm{D}_{21 / 2}-y^{2} \mathrm{G}_{31 / 2}^{\circ} \\
a^{4} \mathrm{~F}_{11 / 2}-y^{2} \mathrm{D}_{11 / 2}^{\circ}\end{array}$ & \\
\hline $\begin{array}{l}\text { 2592. } 32 \\
2590.72 \\
2590.37 \\
2589.70 \\
2589.44\end{array}$ & $\begin{array}{l}2 \\
75 \\
20 w, l \\
30 \\
1\end{array}$ & $\begin{array}{l}38563.96 \\
38587.78 \\
38592.99 \\
38602.97 \\
38606.85\end{array}$ & $\begin{array}{l}c^{4} \mathrm{D}_{21 / 2}-w^{2} \mathrm{~F}_{31 / 2}^{\circ} \\
a^{2} \mathrm{I}_{61 / 2}-z^{2} \mathrm{H}_{51 / 2}^{\circ} \\
a^{2} \mathrm{D}_{21 / 2}-y^{2} \mathrm{D}_{21 / 2}^{\circ}\end{array}$ & $\begin{array}{l}(0.00) 1.03 \\
(0.13) 1.17\end{array}$ \\
\hline $\begin{array}{l}2589.05 \\
2588.25 \\
2587.92 \\
2587.42 \\
2586.98\end{array}$ & $\begin{array}{l}15 \\
12 \\
4 w, l \\
35 \\
3\end{array}$ & $\begin{array}{l}38612.66 \\
38624.60 \\
38630.12 \\
38636.99 \\
38643.56\end{array}$ & $\begin{array}{r}b^{2} \mathrm{~S}_{01 / 2}-w^{2} \mathrm{P}_{11 / 2}^{\circ} \\
a^{4} \mathrm{H}_{41 / 2}-y^{4} \mathrm{H}_{31 / 2}^{\circ}\end{array}$ & $\begin{array}{l}(0.30) 0.98,1.60 \dagger \\
(0.00) 1.18\end{array}$ \\
\hline $\begin{array}{l}2586.69 \\
2585.89 \\
2585.60 \\
2584.83 \\
2584.10\end{array}$ & $\begin{array}{l}\quad 4 \\
2 w \\
15 \\
10 w, l \\
50\end{array}$ & $\begin{array}{l}\text { 38647. } 89 \\
38659.85 \\
\text { 38664. } 33 \\
\text { 38675. } 70 \\
\text { 38686. } 47\end{array}$ & $a^{4} \mathrm{H}_{31 / 2}-y^{4} \mathrm{H}_{31 / 2}^{\circ}$ & $\begin{array}{l}(0.00) 1.25 \dagger \\
(0.00) 0.67 \dagger\end{array}$ \\
\hline
\end{tabular}


TABLE 1. Wavelengths of $\mathrm{Cr}$ II in air-Continued

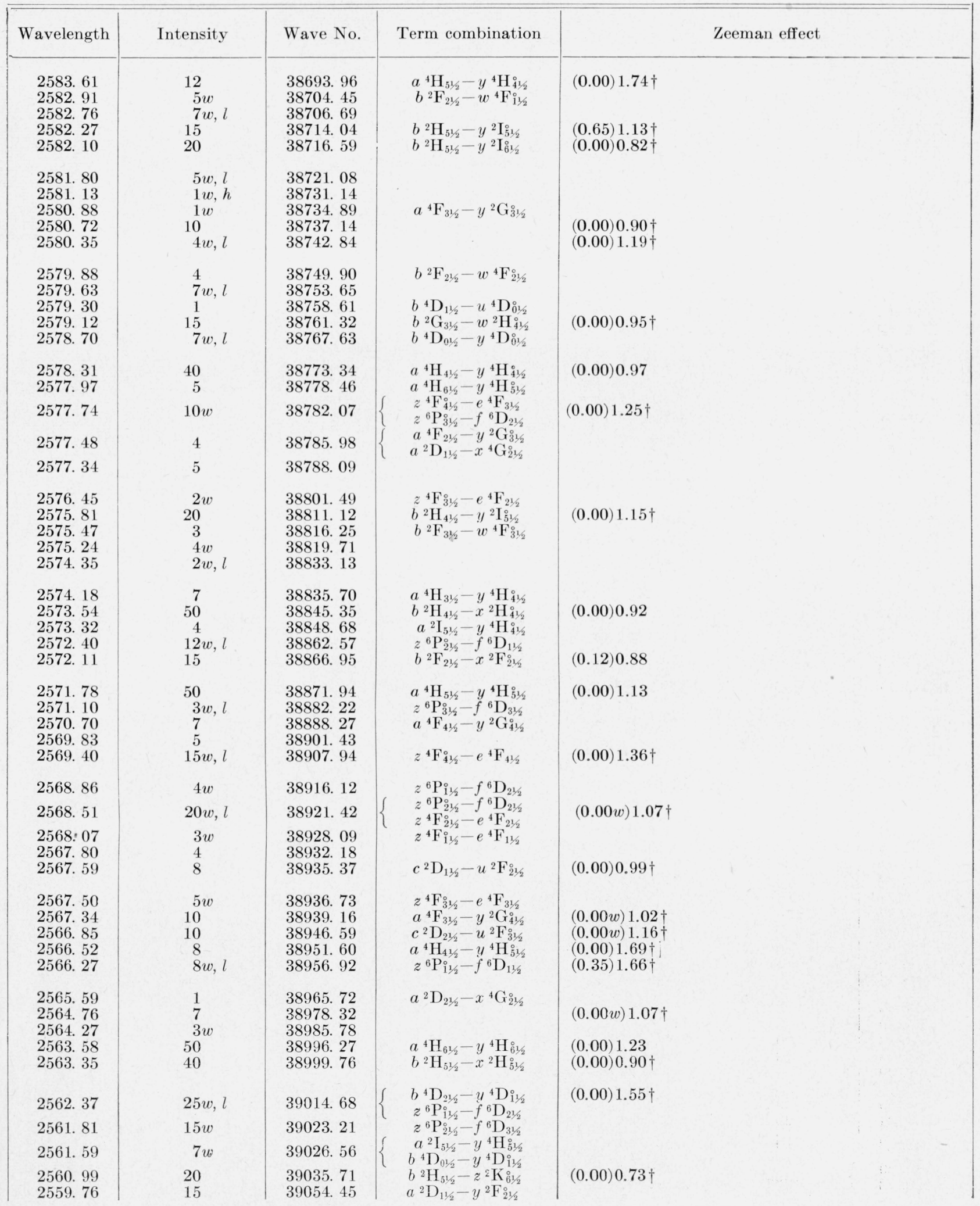


TABlE 1. Wavelengths of $\mathrm{Cr}$ II in air-Continued

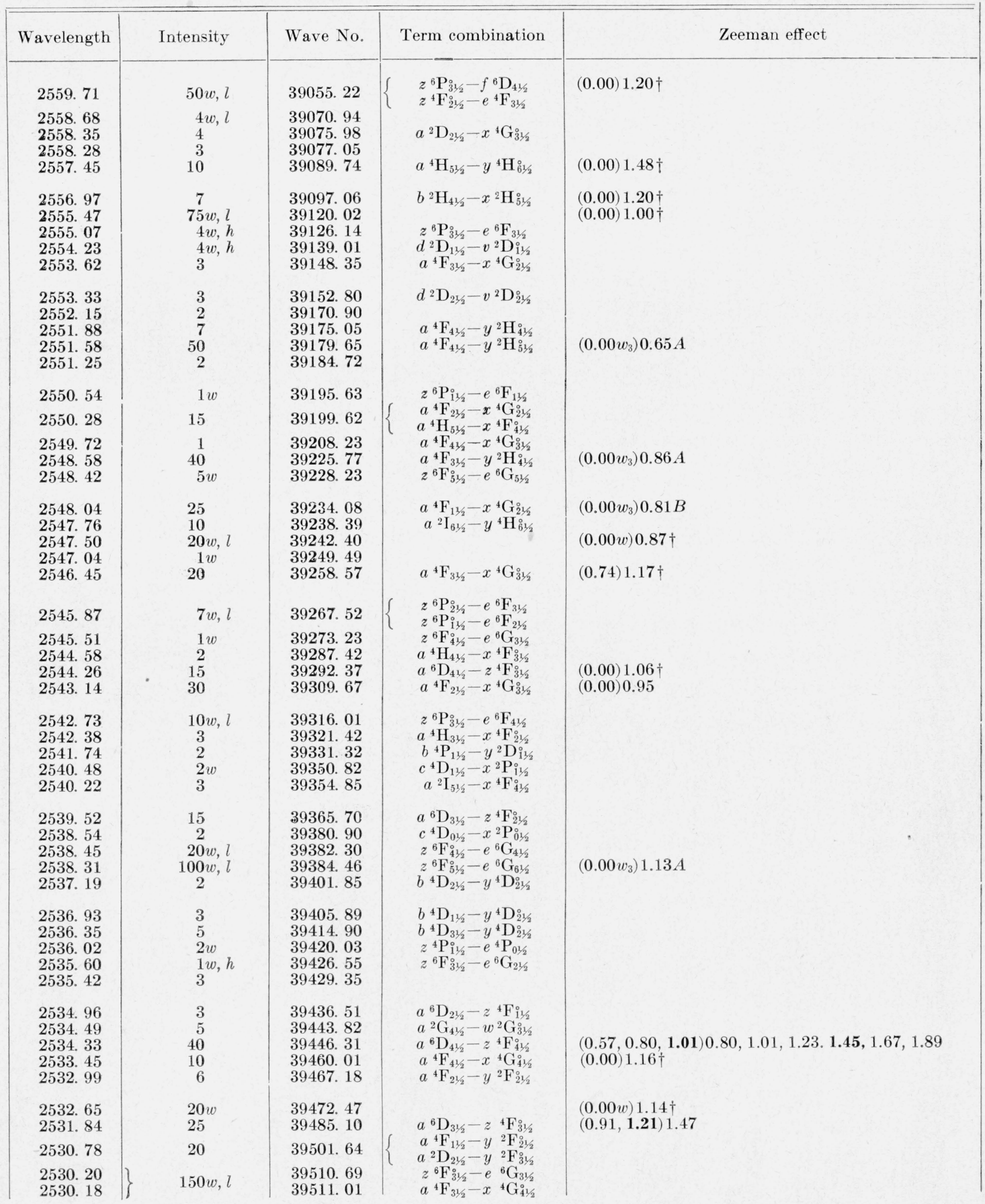


TABLE 1. Wavelengths of Cr II in air-Continued

\begin{tabular}{|c|c|c|c|c|}
\hline Wavelength & Intensity & Wave No. & Term combination & Zeeman effect \\
\hline $\begin{array}{l}2529.90 \\
2529.48 \\
2527.57 \\
2527.40 \\
2526.30\end{array}$ & $\begin{array}{l}75 w, l \\
25 \\
7 \\
2 w, l \\
15 w, l\end{array}$ & $\begin{array}{l}39515.38 \\
39521.94 \\
39551.80 \\
39554.46 \\
39571.68\end{array}$ & $\begin{array}{c}z^{6} \mathrm{~F}_{41 / 2}^{\circ}-e{ }^{6} \mathrm{G}_{51 / 2} \\
a^{6} \mathrm{D}_{21 / 2}-z^{4} \mathrm{~F}_{21 / 2}^{5} \\
a^{6} \mathrm{D}_{11 / 2}-z \\
{ }^{4} \mathrm{~F}_{11 / 2}^{\circ} \\
z^{6} \mathrm{~F}_{21 / 2}^{\circ}-e{ }^{6} \mathrm{G}_{11 / 2}^{\circ} \\
z^{4} \mathrm{P}_{21 / 2}^{\circ}-e{ }^{4} \mathrm{P}_{21 / 2}^{\circ}\end{array}$ & $\begin{array}{l}\left(0.00 w_{3}\right) 1.08 A \\
(0.00) 1.56 \dagger \\
(0.00) 1.67 \dagger\end{array}$ \\
\hline $\begin{array}{l}2525.35 \\
2524.55 \\
2523.93 \\
2523.76 \\
2523.62\end{array}$ & $\begin{array}{l}20 w, l \\
15 w, l \\
15 \\
15 w, l \\
30 w, l\end{array}$ & $\begin{array}{l}\text { 39586. } 57 \\
39599.11 \\
39608.84 \\
39611.50 \\
39613.70\end{array}$ & $\begin{array}{c}a^{2} \mathrm{H}_{51 / 2}-y{ }^{2} \mathbf{I}_{51 / 2}^{\circ} \\
a^{2} \mathrm{H}_{51 / 2}-y{ }^{2} \mathbf{I}_{61 / 2}^{\circ} \\
z^{6} \mathbf{F}_{21 / 2}^{\circ}-e e^{6} \mathrm{G}_{21 / 2}^{\circ}\end{array}$ & $(0.00) 1.33 \dagger$ \\
\hline $\begin{array}{l}2523.24 \\
2522.55 \\
2522.01 \\
2521.76 \\
2521.50\end{array}$ & $\begin{array}{c}150 w, l \\
20 w \\
4 \\
5 \\
1\end{array}$ & $\begin{array}{l}39619.67 \\
39630.51 \\
39638.99 \\
39642.92 \\
39647.01\end{array}$ & 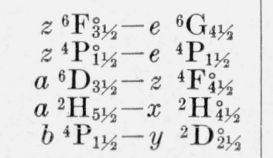 & $\begin{array}{l}\left(0.00 w_{3}\right) 0.98 A \\
(0.00) 1.73 \dagger\end{array}$ \\
\hline $\begin{array}{l}2520.83 \\
2520.65 \\
2520.28 \\
2519.61 \\
2519.08\end{array}$ & $\begin{array}{l}20 w, l \\
40 \\
5 \\
15 w, l \\
25\end{array}$ & $\begin{array}{l}39657.55 \\
39660.38 \\
39666.20 \\
39676.75 \\
39685.10\end{array}$ & $\begin{array}{cc}z^{4} \mathrm{G}_{51 / 2}^{\circ}-f & { }^{4} \mathrm{G}_{41 / 2} \\
a^{4} \mathrm{~F}_{41 / 2}-x & { }^{4} \mathrm{G}_{51 / 2}^{\circ} \\
z^{4} \mathrm{P}_{01 / 2}^{\circ}-e & { }^{4} \mathrm{P}_{01 / 2} \\
a^{4} \mathrm{H}_{41 / 2}-y & { }^{2} \mathrm{G}_{31 / 2}^{\circ}\end{array}$ & $(0.00 w) 0.66 A \dagger$ \\
\hline $\begin{array}{l}2518.84 \\
2518.29 \\
2517.86 \\
2517.36 \\
2516.57\end{array}$ & $\begin{array}{c}30 w, l \\
100 w, l \\
7 w \\
20 w \\
40 w, l\end{array}$ & $\begin{array}{l}39688.87 \\
39697.54 \\
39704.32 \\
39712.21 \\
39724.67\end{array}$ & $\begin{array}{ll}z^{6} \mathrm{~F}_{\mathrm{i} 1 / 2}-e & { }^{6} \mathrm{G}_{11 / 2} \\
z^{6} \mathrm{~F}_{21 / 2}^{1}-e & { }^{6} \mathrm{G}_{31 / 2} \\
z^{4} \mathrm{G}_{51 / 2}^{\circ}-f & { }^{4} \mathrm{G}_{51 / 2}\end{array}$ & $\begin{array}{l}(0.00) 0.79 \dagger \\
(0.00) 1.09 \dagger\end{array}$ \\
\hline $\begin{array}{l}2515.89 \\
2515.06 \\
2513.66 \\
2512.80 \\
2512.38\end{array}$ & $\begin{array}{l}4 \\
55 w, l \\
50 w, l \\
5 w \\
10\end{array}$ & $\begin{array}{l}39735.41 \\
39748.52 \\
39770.65 \\
39784.27 \\
39790.92\end{array}$ & $\begin{array}{l}a{ }^{4} \mathbf{F}_{21 / 2}-y{ }^{2} \mathbf{F}_{31 / 2}^{\circ} \\
z^{6} \mathbf{F}_{11 / 2}^{\circ}-e{ }^{6} \mathrm{G}_{21 / 2}^{\circ} \\
z^{6} \mathbf{F}_{01 / 2}^{\circ}-e{ }^{6} \mathrm{G}_{11 / 2}^{\circ} \\
a^{2} \mathrm{H}_{41 / 2}-y{ }^{2} \mathbf{I}_{51 / 2}^{\circ}\end{array}$ & $\begin{array}{l}(0.00) 0.75 \dagger \\
(0.34) 0.35 \dagger \\
(0.00) 1.02 \dagger\end{array}$ \\
\hline $\begin{array}{l}2512.22 \\
2511.22 \\
2510.24 \\
2509.10 \\
2507.57\end{array}$ & $\begin{array}{l}8 \\
20 \\
20 \\
12 w, l \\
10\end{array}$ & $\begin{array}{l}\text { 39793. } 45 \\
39809.30 \\
39824.83 \\
39842.93 \\
39867.24\end{array}$ & 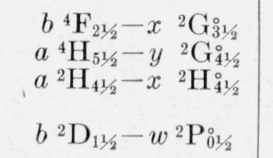 & $\begin{array}{l}(0.00) 1.20 \dagger \\
(0.00) 0.95 \dagger \\
(0.00) 1.59 \dagger \\
(0.00) 0.88 \dagger\end{array}$ \\
\hline $\begin{array}{l}2506.93 \\
2506.76 \\
2506.11 \\
2505.86 \\
2505.45\end{array}$ & $\begin{array}{l}4 \\
5 w \\
8 \\
20 \\
2 w, h\end{array}$ & $\begin{array}{l}39877.42 \\
39880.12 \\
39890.46 \\
39894.44 \\
39900.97\end{array}$ & $\begin{array}{cc}b{ }^{4} \mathrm{D}_{21 / 2}-y & { }^{4} \mathrm{D}_{31 / 2}^{\circ} \\
b & { }^{4} \mathrm{~F}_{31 / 2}-x \\
b^{2} \mathrm{G}_{41 / 2}^{0} \\
b^{4} \mathrm{D}_{31 / 2}-y & { }^{4} \mathrm{D}_{31 / 2}^{\circ} \\
a^{2} \mathrm{H}_{51 / 2}-x & { }^{2} \mathrm{H}_{51 / 2}^{\circ}\end{array}$ & $(0.00) 1.07 \dagger$ \\
\hline $\begin{array}{l}2504.55 \\
2503.89 \\
2503.62 \\
2503.41 \\
2502.96\end{array}$ & $\begin{array}{l}3 w \\
4 w \\
3 \\
2 \\
2 w\end{array}$ & $\begin{array}{l}\text { 39915. } 30 \\
39925.83 \\
\text { 39930. } 13 \\
\text { 39933. } 48 \\
\text { 39940. } 66\end{array}$ & $\begin{array}{l}z^{4} \mathrm{P}_{11 / 2}^{\circ}-e{ }^{4} \mathrm{P}_{21 / 2} \\
a^{2} \mathrm{H}_{51 / 2}-z{ }^{2} \mathrm{~K}_{61 / 2}^{\circ} \\
b^{2} \mathrm{D}_{11 / 2}-w^{2} \mathrm{P}_{11 / 2}\end{array}$ & \\
\hline $\begin{array}{l}2502.16 \\
2501.48 \\
2500.21 \\
2500.07 \\
2499.63\end{array}$ & $\begin{array}{l}12 w \\
25 \\
7 \\
5 \\
5\end{array}$ & $\begin{array}{l}\text { 39953. } 43 \\
\text { 39964. } 29 \\
39984.59 \\
\text { 39986. } 83 \\
\text { 39993. } 87\end{array}$ & 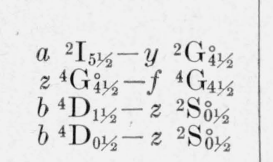 & $\begin{array}{l}(0.00 w) 0.00 w \dagger \\
(0.00) 1.11 \dagger\end{array}$ \\
\hline $\begin{array}{l}2499.35 \\
2498.80 \\
2498.23 \\
2497.87 \\
2496.81\end{array}$ & $\begin{array}{l}8 w \\
40 \\
2 w \\
10 \\
40 w, l\end{array}$ & $\begin{array}{l}39998.34 \\
40007.15 \\
40016.28 \\
40022.04 \\
40039.03\end{array}$ & $\begin{array}{l}a^{4} \mathrm{H}_{61 / 2}-y{ }^{2} \mathrm{H}_{51 / 2}^{\circ} \\
b^{2} \mathrm{D}_{21 / 2}-w^{2} \mathrm{P}_{11 / 2}^{\circ} \\
z^{4} \mathrm{G}_{41 / 2}-f^{4} \mathrm{G}_{51 / 2}\end{array}$ & $\begin{array}{l}(0.00) 1.34 \dagger \\
(0.00) 1.10 \dagger \\
(0.00) 1.15 \dagger\end{array}$ \\
\hline $\begin{array}{l}2496.60 \\
2496.44 \\
2495.20 \\
2495.10 \\
2494.26\end{array}$ & $\begin{array}{l}15 w \\
10 \\
7 w, l \\
7 w, l \\
10 w\end{array}$ & $\begin{array}{l}40042.40 \\
40044.97 \\
40064.87 \\
40066.47 \\
40079.96\end{array}$ & $a^{2} \mathrm{~F}_{21 / 2}-x{ }^{2} \mathrm{G}_{31 / 2}^{\circ}$ & $(0.00) 0.87 \dagger$ \\
\hline
\end{tabular}


TABLE 1. Wavelengths of Cr II in air-Continued

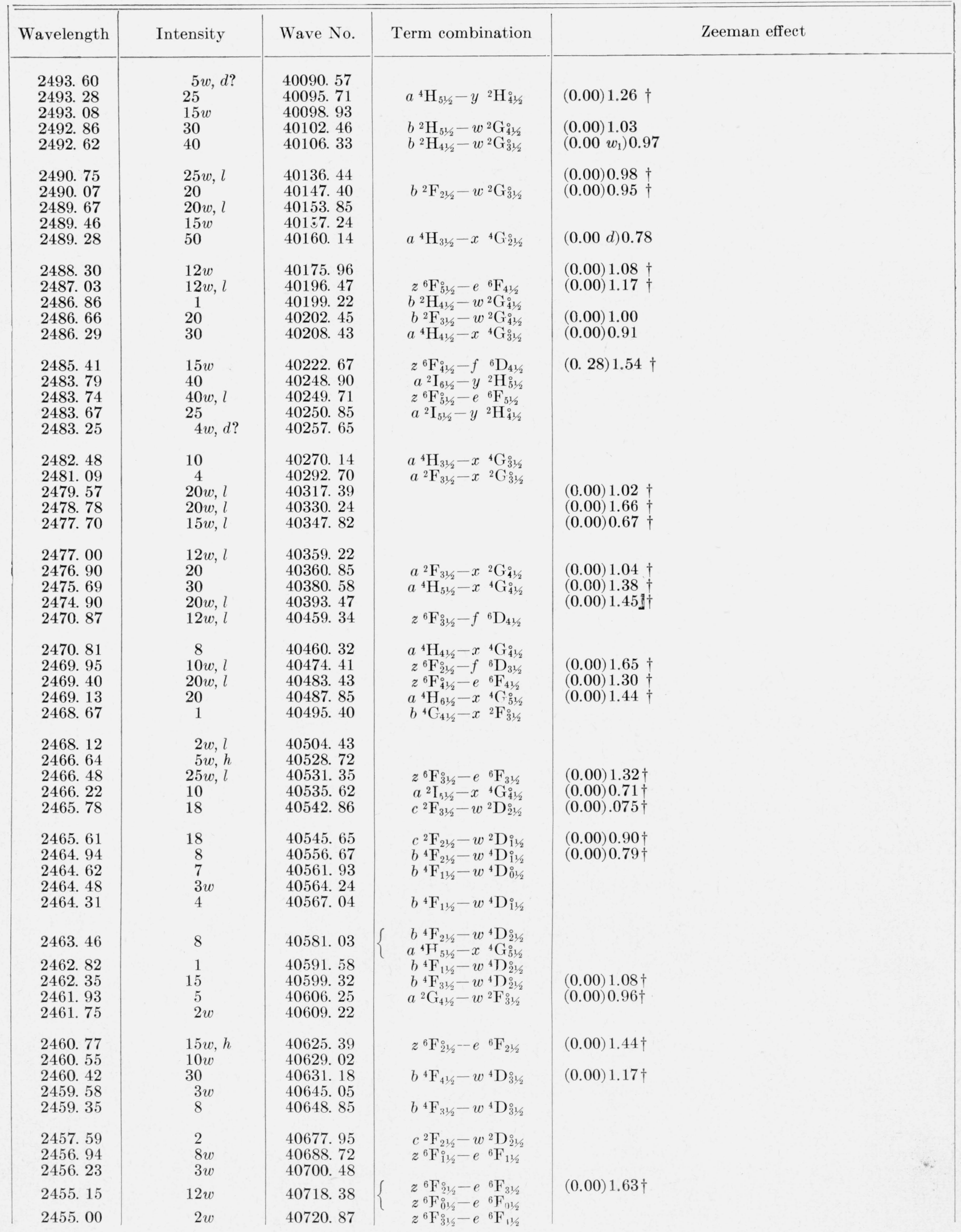


TABLE 1. Wavelengths of $\mathrm{Cr}$ II in air-Continued

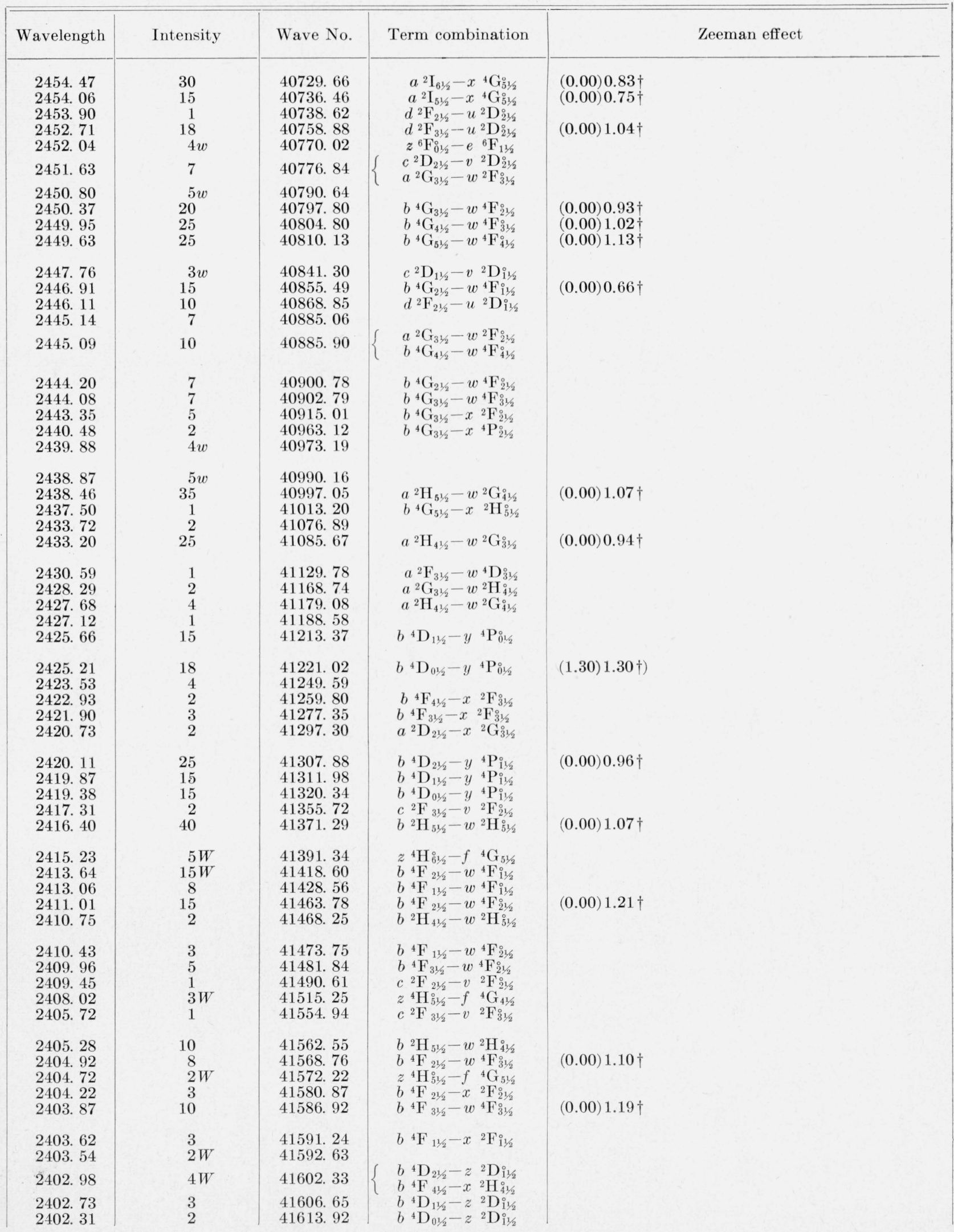


TABLE 1. Wavelengths of $\mathrm{Cr}$ II in air-Continued

\begin{tabular}{|c|c|c|c|c|}
\hline Wavelength & Intensity & Wave No. & Term combination & Zeeman effect \\
\hline $\begin{array}{l}2402.07 \\
2401.33 \\
2400.24 \\
2399.67 \\
2399.21\end{array}$ & $\begin{array}{r}5 \\
2 \\
15 \\
30 \\
3\end{array}$ & $\begin{array}{l}41618.09 \\
41630.90 \\
41649.81 \\
41659.70 \\
41667.69\end{array}$ & $\begin{array}{ll}b & 2 \mathrm{D}_{11 / 2}-u^{2} \mathrm{~F}_{21 / 2}^{\circ} \\
a & { }^{2} \mathrm{P}_{11 / 2}-w^{2} \mathrm{~F}_{21 / 2}^{\circ} \\
b & { }^{4} \mathbf{F}_{41 / 2}-w^{4} \mathbf{F}_{41 / 2}^{\circ} \\
b & { }^{2} \mathrm{H}_{41 / 2}-w^{2} \mathbf{H}_{41 / 2} \\
b & { }^{4} \mathbf{F}_{31 / 2}-w^{4} \mathrm{~F}_{41 / 2}^{\circ 1}\end{array}$ & $\begin{array}{l}(0.00) 1.30 \dagger \\
(0.00) 0.90 \dagger\end{array}$ \\
\hline $\begin{array}{l}2398.51 \\
2398.28 \\
2397.75 \\
2396.48 \\
2393.99\end{array}$ & $\begin{array}{r}15 \\
1 \\
40 \\
10 \\
50\end{array}$ & $\begin{array}{l}41679.85 \\
41683.84 \\
41693.06 \\
41715.15 \\
41758.53\end{array}$ & $\begin{array}{lll}b & { }^{4} \mathrm{D}_{21 / 2}-y & { }^{4} \mathrm{P}_{21 / 2} \\
b & { }^{4} \mathrm{D}_{11 / 2}-y & { }^{4} \mathrm{P}_{21 / 2}^{2} \\
b & { }^{4} \mathrm{D}_{31 / 2}-y & { }^{4} \mathrm{P}_{21 / 2}^{\circ} \\
a^{2} & { }^{2} \mathbf{F}_{21 / 2}-w^{4} \mathbf{F}_{21 / 2}^{\circ} \\
a^{2} \mathbf{F}^{21 / 2}-x & { }^{2} \mathbf{F}_{31 / 2}^{01}\end{array}$ & $\begin{array}{l}(0.00) 1.32 \dagger \\
(0.00) 1.16 \dagger\end{array}$ \\
\hline $\begin{array}{l}2393.35 \\
2392.80 \\
2392.55 \\
2389.75 \\
2387.03\end{array}$ & $\begin{array}{r}4 \\
4 \\
5 \\
40 \\
4\end{array}$ & $\begin{array}{l}41769.70 \\
41779.30 \\
41783.67 \\
41832.62 \\
41880.28\end{array}$ & $\begin{array}{l}b{ }^{2} \mathrm{D}_{21 / 2}-u{ }^{2} \mathrm{~F}_{31 / 2}^{\circ} \\
a^{2} \mathbf{F}_{21 / 2}-x{ }^{2} \mathrm{~F}_{21 / 2}^{\circ} \\
a^{2} \mathrm{D}_{11 / 2}-w^{4} \mathrm{D}_{11 / 2}^{\circ}\end{array}$ & $(0.00) 0.88 \dagger$ \\
\hline $\begin{array}{l}2386.08 \\
2382.20 \\
2381.97\end{array}$ & $\begin{array}{l}3 \\
5 \\
2\end{array}$ & $\begin{array}{l}\text { 41896. } 96 \\
41965.19 \\
41969.24\end{array}$ & 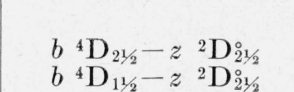 & \\
\hline 2381. 48 & 50 & 41977. 88 & $\left\{\begin{array}{l}a^{4} \mathrm{P}_{01 / 2}-y{ }^{4} \mathrm{D}_{01 / 2}^{0} \\
a^{4} \mathrm{P}^{11}-y-y{ }^{4} \mathrm{D}_{01}^{0}\end{array}\right.$ & $\mathrm{P}-\mathrm{B}$ \\
\hline 2378. 90 & 3 & 42023. 40 & $b{ }^{4} \mathbf{D}_{21 / 2}^{1 / 2}-z{ }^{2} \mathbf{P}_{11 / 2}^{0 / 2}$ & \\
\hline $\begin{array}{l}2378.68 \\
2378.28 \\
2377.32 \\
2376.40 \\
2375.69\end{array}$ & $\begin{array}{l}5 \\
3 \\
2 \\
5 \\
4\end{array}$ & $\begin{array}{l}42027.29 \\
42034.36 \\
42051.32 \\
42067.60 \\
42080.17\end{array}$ & 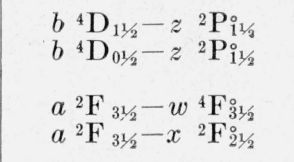 & \\
\hline $\begin{array}{l}2374.57 \\
2373.70 \\
2372.63\end{array}$ & $\begin{array}{l}1 \\
2 \\
2\end{array}$ & $\begin{array}{l}42100.02 \\
42115.45 \\
42134.44\end{array}$ & 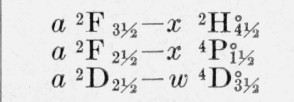 & \\
\hline 2371. 23 & 4 & 42159. 32 & & \\
\hline 2366.84 & $35 w$ & 42237.50 & $\left\{\begin{array}{l}a{ }^{4} \mathrm{P}_{01 / 2}-y^{4} \mathrm{D}_{11 / 2}^{10} \\
a^{4} \mathrm{P}_{11 / 2}-y^{4} \mathrm{D}_{11 / 2}^{0}\end{array}\right.$ & $\mathrm{P}-\mathrm{B}$ \\
\hline $\begin{array}{l}\text { 2366. } 75 \\
\text { 2366. } 28\end{array}$ & $\begin{array}{l}5 \\
1\end{array}$ & $\begin{array}{l}42239.12 \\
42248.04\end{array}$ & $\begin{array}{l}a^{4} \mathrm{P}_{21 / 2}-y^{4} \mathrm{D}_{11 / 2}^{\circ} \\
a^{2} \mathrm{H}_{41 / 2}-w^{2} \mathrm{~F}_{31 / 2}^{\circ}\end{array}$ & \\
\hline 2365. 26 & $20 w$ & 42265. 72 & $\left\{\begin{array}{l}a^{2} \mathrm{H}_{51 / 2}-w^{2} \mathrm{H}_{51 / 2}^{0} \\
a^{4} \mathrm{~F}_{41 / 3}-w^{4} \mathrm{D}_{31 / 2}^{0}\end{array}\right.$ & $(0.00) 1.13 \dagger$ \\
\hline $\begin{array}{l}2365.15 \\
2364.98\end{array}$ & $\begin{array}{l}4 \\
2\end{array}$ & $\begin{array}{l}\text { 42267. } 68 \\
42270.72\end{array}$ & $a_{2}{ }^{4} \mathrm{~F}_{31 / 2}-w^{4}{ }^{4} \mathrm{D}_{21 / 2}^{2}$ & \\
\hline $\begin{array}{l}2364.02 \\
2363.65 \\
2363.32 \\
2362.26 \\
2362.00\end{array}$ & $\begin{array}{l}10 \\
3 \\
1 w \\
2 \\
1\end{array}$ & $\begin{array}{l}42287.89 \\
42294.50 \\
42300.41 \\
42319.39 \\
42324.05\end{array}$ & $\begin{array}{l}a{ }^{6} \mathrm{D}_{41 / 2}-z^{4} \mathrm{D}_{31 / 2}^{\circ} \\
a^{4} \mathrm{~F}_{21 / 2}-w^{4} \mathrm{D}_{11 / 2}^{12} \\
a^{4} \mathrm{~F}_{21 / 2}-w^{4} \mathrm{D}_{21 / 2}^{\circ 1} \\
a^{4} \mathrm{~F}_{11 / 2}-w^{4} \mathrm{D}_{01 / 2}^{0}\end{array}$ & \\
\hline $\begin{array}{l}2361.79 \\
2361.31 \\
2361.09\end{array}$ & $\begin{array}{l}3 \\
1 \\
1\end{array}$ & $\begin{array}{l}42327.81 \\
42336.41 \\
42340.35\end{array}$ & $\begin{array}{l}b^{2} \mathbf{F}_{31 / 2}-x^{2} \mathrm{D}_{21 / 2}^{\circ} \\
b^{4} \mathrm{D}_{11 / 2}-y^{4} \mathbf{F}_{11 / 2}^{\circ} \\
b^{4} \mathrm{D}_{21 / 2}-y{ }^{4} \mathbf{F}_{21 / 2}^{\circ}\end{array}$ & \\
\hline 2360.89 & $6 l$ & 42343.94 & $\begin{array}{l}b^{4} \mathrm{D}_{01 / 2}-y^{4} \mathrm{~F}_{11 / 2}^{\circ} \\
b^{4} \mathrm{D}_{11}-y^{4} \mathrm{~F}_{21 / 2}^{\circ}\end{array}$ & \\
\hline 2360.75 & 8 & 42346. 46 & $b^{4} \mathrm{D}_{21 / 2}^{11 / 2}-y^{4} \mathbf{F}_{31 / 2}^{2 / 2}$ & \\
\hline $\begin{array}{l}2360.14 \\
2358.82 \\
2356.96 \\
2356.58 \\
2355.62\end{array}$ & $\begin{array}{r}10 \\
5 \\
5 \\
4 \\
3\end{array}$ & $\begin{array}{l}42357.40 \\
42381.10 \\
42414.54 \\
42421.38 \\
42438.67\end{array}$ & $\begin{array}{l}a^{2} \mathrm{P}_{11 / 2}-x^{2} \mathrm{P}_{11 / 2}^{\circ} \\
a^{2} \mathrm{~F}_{21 / 2}-y^{2} \mathrm{P}_{11 / 2}^{\circ} \\
b^{4} \mathrm{D}_{31 / 2}-y^{4} \mathrm{~F}_{41 / 2}^{\circ} \\
a^{2} \mathrm{P}_{11 / 2}-x^{2} \mathrm{P}_{01 / 2}^{\circ} \\
a^{2} \mathbf{S}_{01 / 2}-w^{2} \mathbf{P}_{01 / 2}^{\circ}\end{array}$ & \\
\hline $\begin{array}{l}2355.10 \\
2354.64 \\
2354.59 \\
2354.05 \\
2353.54\end{array}$ & $\begin{array}{l}3 \\
3 \\
3 \\
3 \\
1\end{array}$ & $\begin{array}{l}42448.04 \\
42456.33 \\
42457.23 \\
42466.97 \\
42476.17\end{array}$ & $\begin{array}{l}a^{2} \mathrm{H}_{41 / 2}-w^{2} \mathrm{H}_{51 / 2}^{\circ} \\
a^{6} \mathrm{D}_{01 / 2}-z^{4} \mathrm{D}_{01 / 2}^{0} \\
a^{2} \mathrm{H}_{51 / 2}-w^{2} \mathrm{H}_{41 / 2}^{0} \\
a^{6} \mathrm{D}_{11 / 2}-z^{4} \mathrm{D}_{11 / 3}^{0}\end{array}$ & \\
\hline $\begin{array}{l}2353.44 \\
2353.29 \\
2351.96 \\
2350.14 \\
2350.00\end{array}$ & $\begin{array}{l}3 \\
3 \\
4 \\
1 \\
2\end{array}$ & $\begin{array}{l}42477.98 \\
42480.68 \\
42504.71 \\
42537.62 \\
42540.15\end{array}$ & $\begin{array}{c}a^{6} \mathrm{D}_{21 / 2}-z^{4} \mathrm{D}_{21 / 2}^{\circ} \\
a^{6} \mathrm{D}_{31 / 2}-z^{4} \mathrm{D}_{31 / 2}^{0} \\
a^{2} \mathrm{~S}_{01 / 2}-w^{2} \mathrm{P}_{11 / 2}^{0} \\
a^{6} \mathrm{D}_{01 / 2}-z^{4} \mathrm{D}_{11 / 2}^{1} \\
b^{2} \mathrm{~F}_{21 / 2}-x^{2} \mathrm{D}_{11 / 2}^{\circ}\end{array}$ & i \\
\hline
\end{tabular}


TABLE 1. Wavelengths of $\mathrm{Cr}$ I in air-Continued

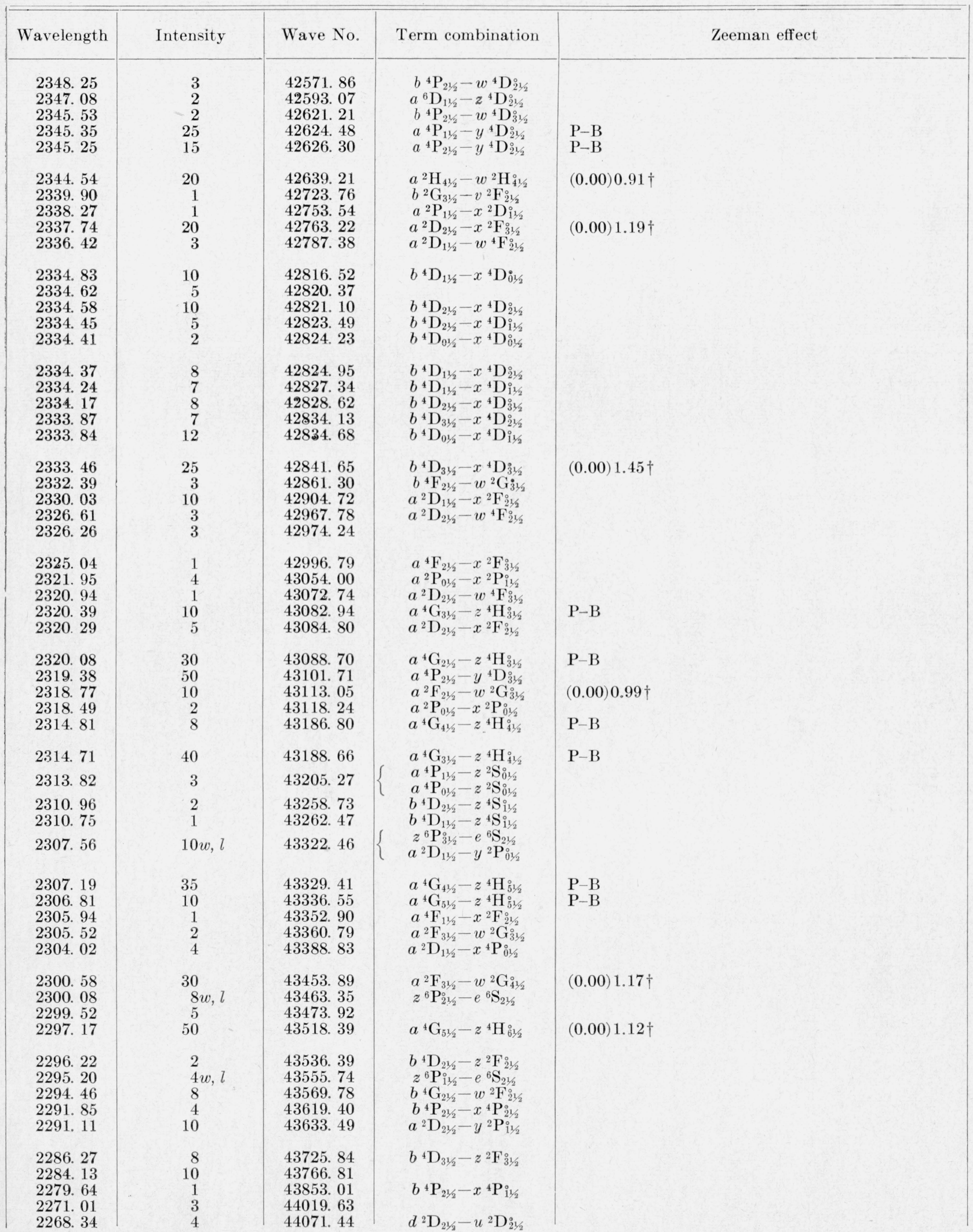


TABLE 1. Wavelengths of $\mathrm{Cr}$ II in air-Continued

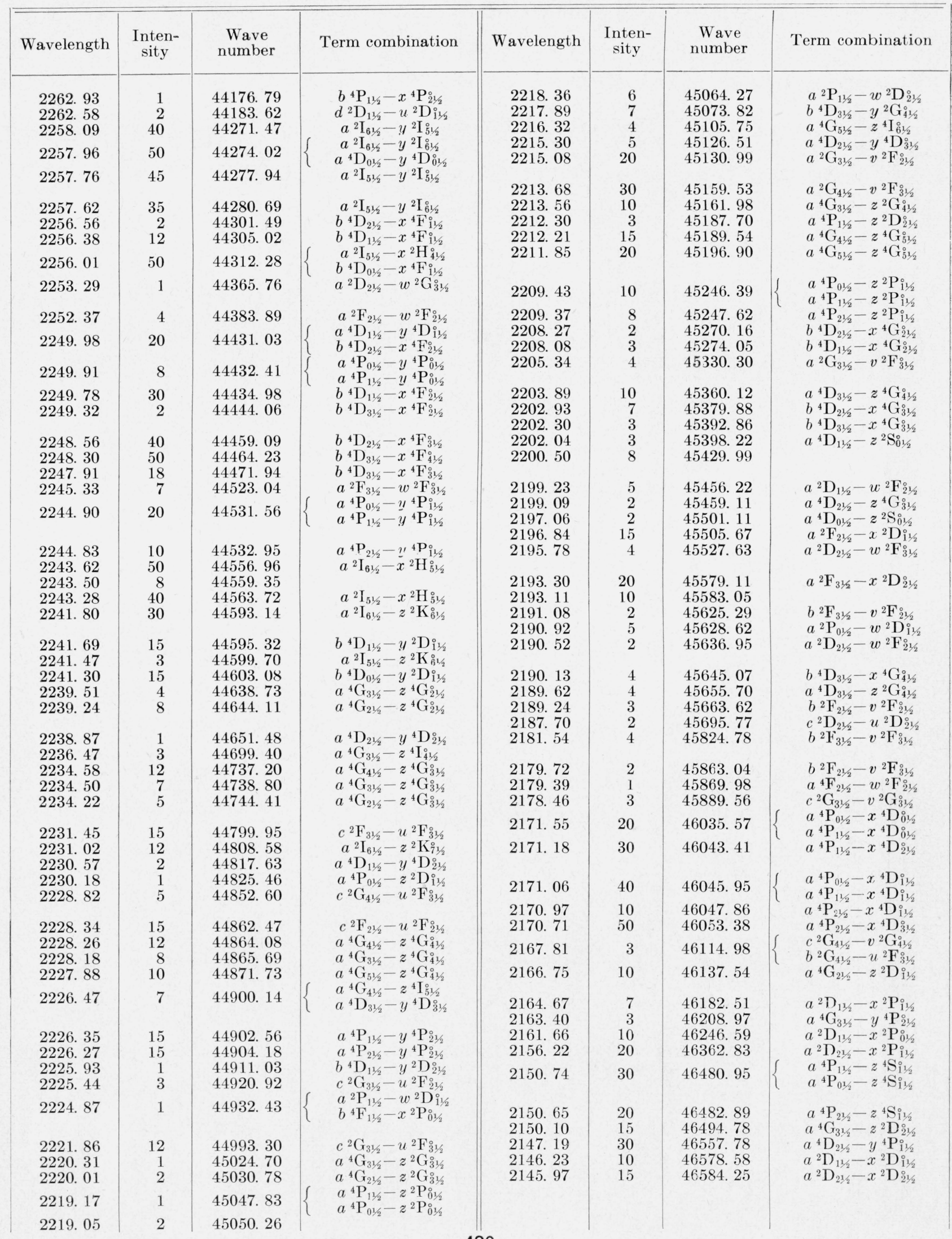


TABLE 1. Wavelengths of $\mathrm{Cr}$ II in air-Continued

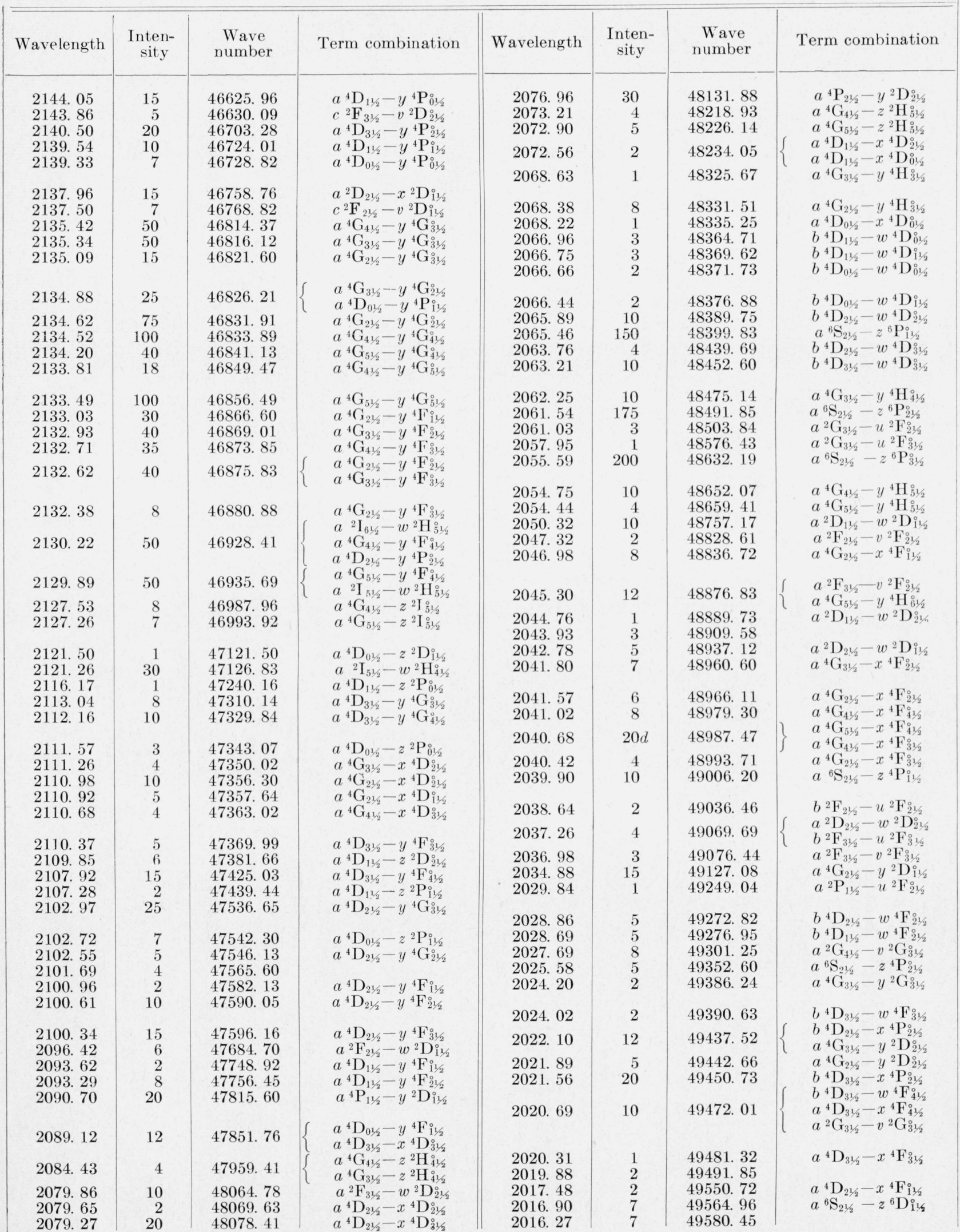


TABLE 1. Wavelengths of $\mathrm{Cr}$ II in air-Continued

\begin{tabular}{|c|c|c|c|c|c|c|c|}
\hline Wavelength & $\begin{array}{l}\text { Inten- } \\
\text { sity }\end{array}$ & $\begin{array}{l}\text { Wave } \\
\text { number }\end{array}$ & Term combination & Wavelength & $\begin{array}{l}\text { Inten- } \\
\text { sity }\end{array}$ & Wave No. & Term combination \\
\hline $\begin{array}{l}2015.87 \\
2013.65 \\
2012.74 \\
2012.58 \\
2012.43\end{array}$ & $\begin{array}{l}15 \\
40 \\
10 \\
20 \\
10\end{array}$ & $\begin{array}{l}49590.29 \\
49644.95 \\
49667.39 \\
49671.34 \\
49675.04\end{array}$ & $\begin{array}{l}a^{4} \mathrm{G}_{31 / 2}-y^{2} \mathrm{G}_{41 / 2}^{0} \\
a^{6} \mathrm{~S}_{21 / 2}-z^{6} \mathrm{D}_{31 / 2}^{0} \\
a^{2} \mathrm{G}_{41 / 2}-v^{2} \mathrm{G}_{41 / 2}^{0} \\
b^{4} \mathrm{D}_{21 / 2}-x^{4} \mathrm{P}_{11 / 2}^{0} \\
b^{4} \mathrm{D}_{11 / 2}-x^{4} \mathrm{P}_{11 / 2}^{0}\end{array}$ & $\begin{array}{l}\text { 2004. } 24 \\
2004.03 \\
2003.88 \\
2002.99 \\
2002.71\end{array}$ & $\begin{array}{r}10 \\
5 \\
35 \\
30 \\
10\end{array}$ & $\begin{array}{l}49878.00 \\
49883.22 \\
49886.96 \\
49909.12 \\
49916.10\end{array}$ & $\begin{array}{l}b^{4} \mathrm{D}_{11 / 2}-x^{4} \mathrm{P}_{01 / 2}^{\circ} \\
a^{4} \mathrm{G}_{51 / 2}-y^{2} \mathrm{H}_{41 / 2}^{\circ} \\
a^{4} \mathrm{G}_{51 / 2}-y^{2} \mathrm{H}_{51 / 2}^{\circ} \\
a^{4} \mathrm{G}_{31 / 2}-x^{4} \mathrm{G}_{31 / 2}^{\circ} \\
a^{4} \mathrm{G}_{21 / 2}-x^{4} \mathrm{G}_{31 / 2}^{\circ}\end{array}$ \\
\hline $\begin{array}{l}\text { 2012. } 21 \\
\text { 2012. } 12\end{array}$ & $\begin{array}{r}25 \\
4\end{array}$ & $\begin{array}{l}49680.47 \\
49682.69\end{array}$ & $\begin{array}{l}a^{4} \mathrm{D}_{21 / 2}-x{ }^{4} \mathrm{~F}_{21 / 2}^{0} \\
b^{4} \mathrm{D}_{01 / 2}-x{ }^{4} \mathrm{P}_{11 / 2}^{\circ}\end{array}$ & $\begin{array}{l}\text { 2001. } 65 \\
\text { 2001. } 36\end{array}$ & $\begin{array}{l}4 \\
3\end{array}$ & $\begin{array}{l}49942.53 \\
49949.76\end{array}$ & $\begin{array}{l}b{ }^{4} \mathrm{D}_{11 / 2}-y{ }^{2} \mathrm{P}_{11 / 2} \\
b^{4} \mathrm{D}_{01 / 2}-y^{2} \mathrm{P}_{11 / 2}^{\circ}\end{array}$ \\
\hline 2011. 13 & 20 & 49707. 14 & $\begin{array}{l}a^{6} \mathrm{~S}_{21 / 2}-z^{6} \mathrm{D}_{21 / 2}^{0} \\
a^{4} \mathrm{D}_{21}-x^{4} \mathrm{~F}^{0}\end{array}$ & 2000.76 & 5 & 4006474 & $b^{2} \mathrm{H}-v^{2} \mathrm{G}:$ \\
\hline $\begin{array}{l}\text { 2007. } 39 \\
\text { 2007. } 18\end{array}$ & $\begin{array}{l}10 \\
20\end{array}$ & $\begin{array}{l}\text { 49799. } 74 \\
49804.95\end{array}$ & $\begin{array}{l}a^{4} \mathrm{G}_{31 / 2}-x{ }^{4} \mathrm{G}_{21 / 2}^{21 / 2} \\
a^{4} \mathrm{G}_{21 / 2}-x^{4} \mathrm{G}_{21 / 2}^{2}\end{array}$ & & & & \\
\hline 2006. 91 & 10 & 49811. 65 & $b{ }^{4} \mathrm{D}_{11 / 2}-y^{2} \mathrm{P}_{01 / 2}^{\circ}$ & & & & \\
\hline 2006. 61 & 10 & 49819. 10 & $\begin{array}{l}b^{4} \mathrm{D}_{01 / 2}-y^{2} \mathrm{P}_{01 / 2}^{012} \\
a^{4} \mathrm{D}_{016}-x^{4} \mathrm{~F}^{0} 11\end{array}$ & & & & \\
\hline $\begin{array}{l}\text { 2005. } 50 \\
2005.19\end{array}$ & $\begin{array}{l}4 \\
3\end{array}$ & $\begin{array}{l}\text { 49846. } 67 \\
49854.37\end{array}$ & $a{ }^{4} \mathrm{D}_{11 / 2}-x{ }^{4} \mathrm{~F}_{21 / 2}^{21}$ & & & & \\
\hline 2004. 34 & 35 & 49875.51 & $\begin{array}{l}a^{4} \mathrm{G}_{41 / 2}-y^{2} \mathrm{H}_{41 / 2}^{\circ} \\
a^{4} \mathrm{G}_{31 / 2}-y^{2} \mathrm{H}_{41 / 2}^{0}\end{array}$ & & & & \\
\hline
\end{tabular}

TABLE 2. Wavelengths of $\mathrm{Cr}$ II in vacuum

\begin{tabular}{|c|c|c|c|c|c|c|c|}
\hline Wavelength & $\begin{array}{l}\text { Inten- } \\
\text { sity }\end{array}$ & $\begin{array}{l}\text { Wave } \\
\text { number }\end{array}$ & Term combination & Wavelength & $\begin{array}{l}\text { Inten- } \\
\text { sity }\end{array}$ & $\begin{array}{c}\text { Wave } \\
\text { number }\end{array}$ & Term combination \\
\hline $\begin{array}{l}1998.14 \\
1996.62 \\
1993.63 \\
1993.37 \\
1990.79 \\
1987.43 \\
1985.67 \\
1985.52 \\
1963.00 \\
1955.93 \\
1950.06 \\
1949.22 \\
1949.00 \\
1948.51 \\
1945.98 \\
1939.96 \\
1939.15 \\
1938.42 \\
1937.56 \\
1935.58 \\
1932.64 \\
1929.96 \\
1923.02 \\
1918.30 \\
1911.36 \\
1907.36 \\
1898.92 \\
1890.55 \\
1887.96 \\
1884.12 \\
1883.35 \\
1881.06 \\
1879.05 \\
1875.22 \\
1870.46\end{array}$ & $\begin{array}{r}2 \\
3 \\
25 \\
15 \\
10 \\
\\
5 \\
12 \\
22 \\
15 \\
15 \\
\\
50 \\
35 \\
40 \\
10 \\
10 \\
\\
5 \\
6 \\
3 \\
20 \\
25 \\
\\
5 \\
12 \\
8 \\
4 \\
7 \\
3 \\
35 \\
30 \\
6 \\
1 \\
10 \\
6 \\
10 \\
3 \\
1\end{array}$ & $\begin{array}{l}50046.54 \\
50084.54 \\
50159.81 \\
50166.40 \\
50231.22 \\
50316.24 \\
50360.92 \\
50364.69 \\
50942.56 \\
51126.52 \\
\\
51280.39 \\
51302.57 \\
51308.42 \\
51321.32 \\
51388.49 \\
\\
51549.02 \\
51568.99 \\
51588.51 \\
51611.30 \\
51664.02 \\
\\
51742.66 \\
51814.65 \\
52001.43 \\
52129.39 \\
52318.88 \\
52428.49 \\
52661.51 \\
52894.72 \\
52967.30 \\
53075.18 \\
53096.88 \\
53161.41 \\
53218.38 \\
53327.08 \\
53462.64\end{array}$ & 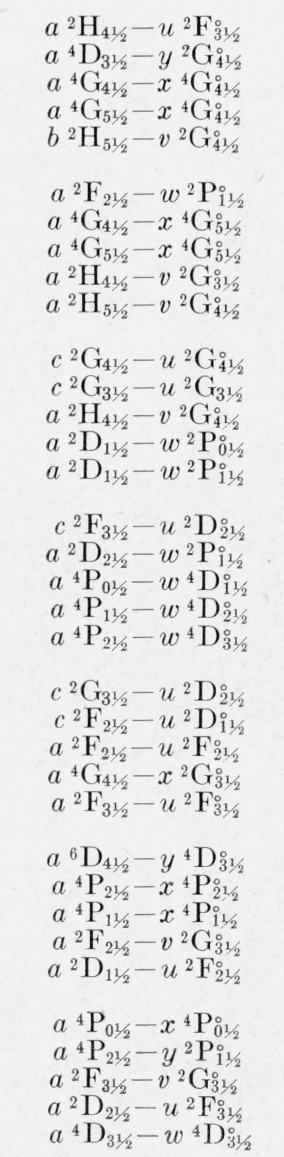 & $\begin{array}{l}\text { 1866. } 32 \\
1865.80 \\
1860.12 \\
1858.72 \\
1858.44 \\
1855.14 \\
1854.68 \\
1854.46 \\
1852.37 \\
1852.13 \\
1836.23 \\
1830.61 \\
1828.62 \\
1825.34 \\
1823.07 \\
1821.58 \\
1820.84 \\
1819.81 \\
1818.89 \\
1815.32 \\
1813.50 \\
1812.95 \\
1810.08 \\
1808.66 \\
1791.51 \\
1786.07\end{array}$ & $\begin{array}{r}15 \\
1 \\
12 \\
15 \\
20 \\
\\
20 \\
3 \\
1 \\
3 \\
25 \\
12 \\
5 \\
3 \\
3 \\
1 \\
8 \\
4 \\
5 \\
1 \\
3 \\
1 \\
1 \\
5 \\
2 \\
2 \\
2\end{array}$ & $\begin{array}{l}53581.26 \\
53596.31 \\
53759.85 \\
53800.52 \\
53808.43 \\
\\
53904.35 \\
53917.75 \\
53924.05 \\
53984.96 \\
53991.80 \\
\\
54459.44 \\
54626.66 \\
54686.05 \\
54784.16 \\
54852.53 \\
\\
54897.25 \\
54919.83 \\
54950.79 \\
54978.53 \\
55086.56 \\
55141.99 \\
55158.60 \\
55246.10 \\
55289.68 \\
55818.86 \\
55988.99\end{array}$ & 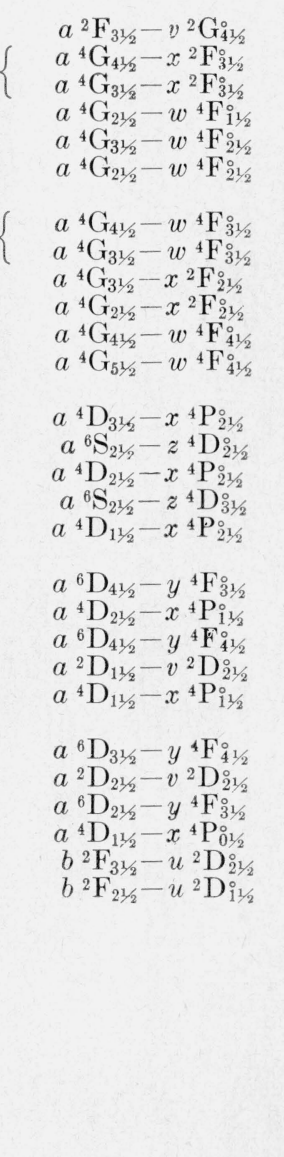 \\
\hline
\end{tabular}


TABLE 3. Even terms of $\mathrm{Cr}$ II

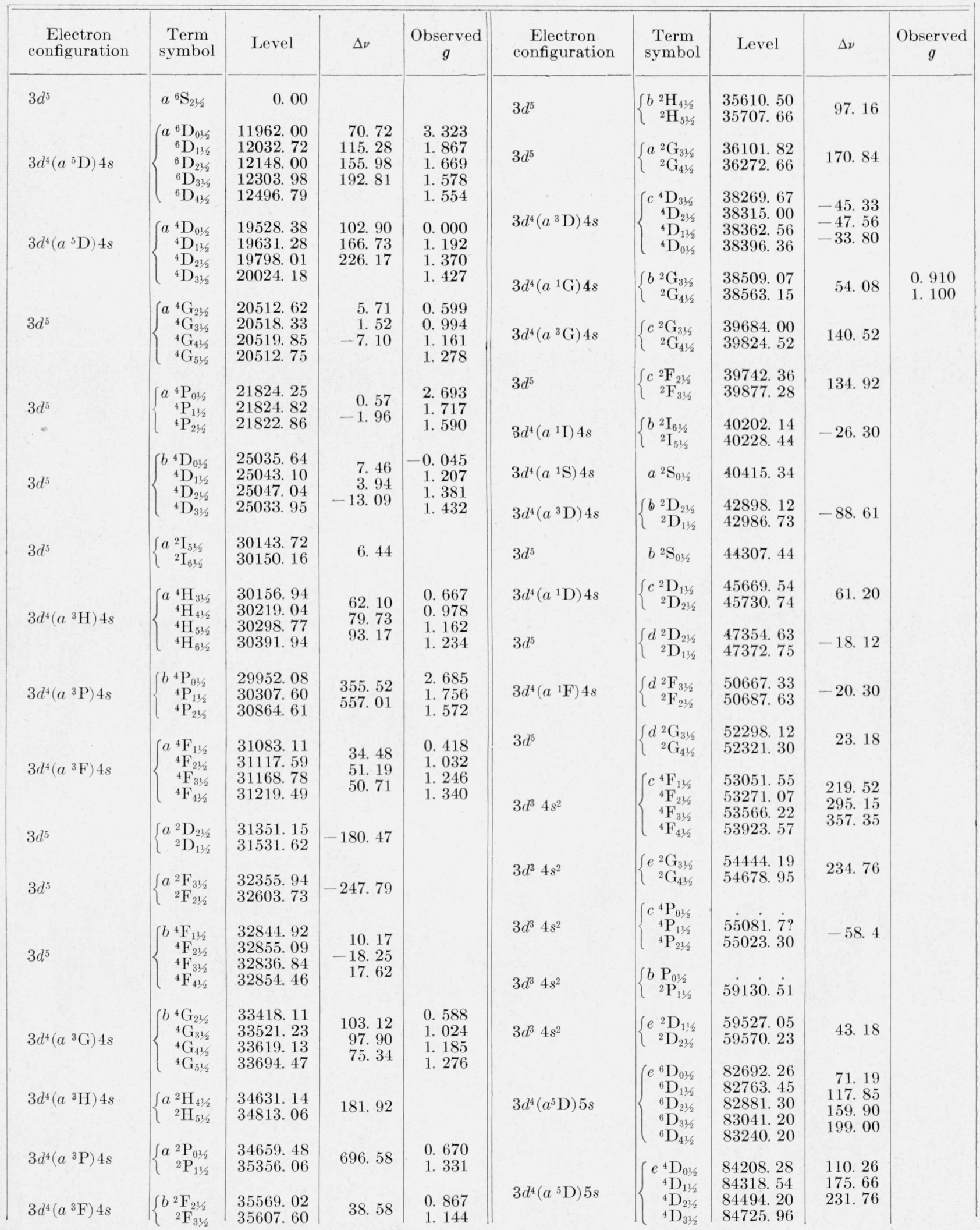


TABLE 3. Even terms of $\mathrm{Cr} \mathrm{II}$-Continued

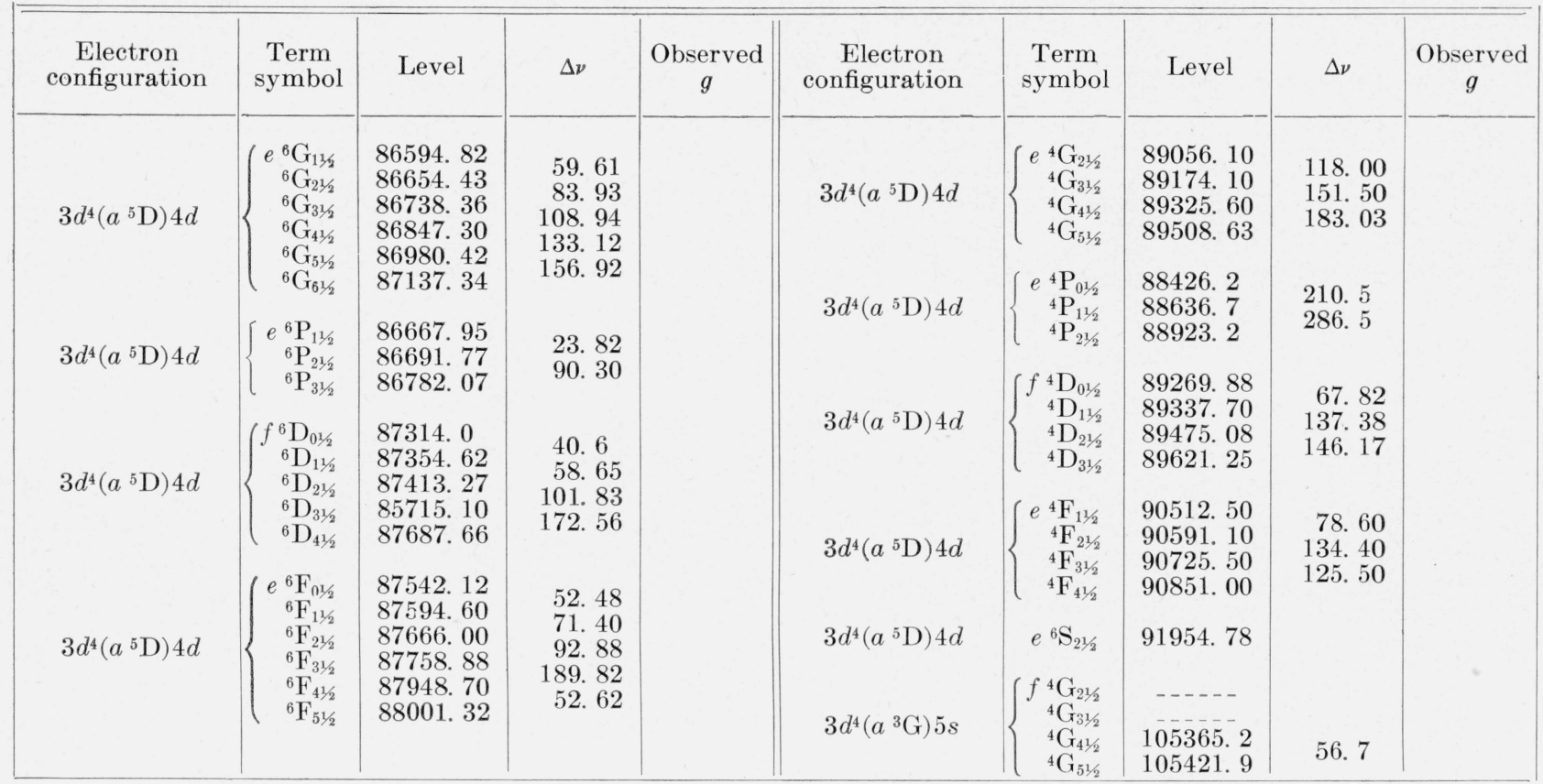

Table 4. Odd terms of $\mathrm{Cr}$ II

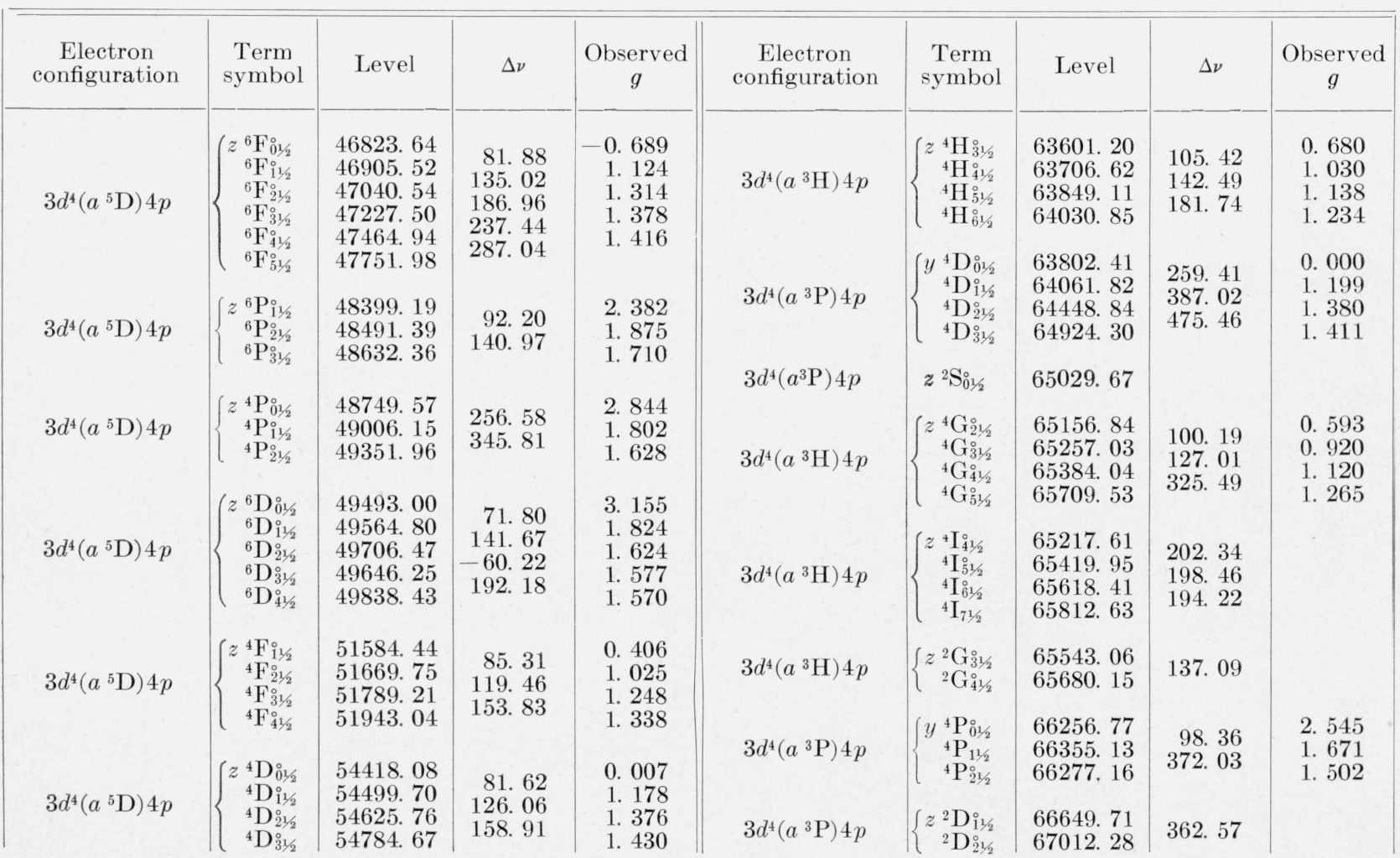


TABLE 4. Odd terms of $\mathrm{Cr} \mathrm{II}-$ Continued

\begin{tabular}{|c|c|c|c|c|c|c|c|c|c|}
\hline $\begin{array}{l}\text { Electron } \\
\text { configuration }\end{array}$ & $\begin{array}{c}\text { Term } \\
\text { symbol }\end{array}$ & Level & $\Delta \nu$ & $\begin{array}{c}\text { Observed } \\
g\end{array}$ & $\begin{array}{c}\text { Electron } \\
\text { configuration }\end{array}$ & $\begin{array}{c}\text { Term } \\
\text { symbol }\end{array}$ & Level & $\Delta \nu$ & $\begin{array}{c}\text { Observed } \\
g\end{array}$ \\
\hline $3 d^{4}\left(a^{3} \mathrm{P}\right) 4 p$ & $\left\{\begin{array}{c}z^{2} \mathrm{P}_{01 / 2}^{\circ} \\
{ }^{2} \mathbf{P}_{11 / 2}^{\circ}\end{array}\right.$ & $\begin{array}{l}66872.12 \\
67070.48\end{array}$ & 198. 36 & & $3 d^{4}\left(a^{3} \mathrm{D}\right) 4 p$ & $\left\{\begin{array}{r}x^{2} \mathrm{~F}_{31 / 2}^{\circ} \\
{ }^{2} \mathbf{F}_{21 / 2}^{\circ}\end{array}\right.$ & $\begin{array}{l}\text { 74114. } 48 \\
74436.14\end{array}$ & -321.66 & \\
\hline $3 d^{4}\left(a^{3} \mathrm{~F}\right) 4 p$ & $\left\{\begin{array}{r}y^{4} \mathrm{G}_{21 / 2}^{\circ} \\
{ }^{4} \mathrm{G}_{31 / 2}^{\circ} \\
{ }^{4} \mathrm{G}_{41 / 2}^{\circ} \\
{ }^{4} \mathrm{G}_{51 / 2}^{\circ}\end{array}\right.$ & $\begin{array}{l}67344.42 \\
67334.20 \\
67353.60 \\
67369.33\end{array}$ & $\begin{array}{r}-10.22 \\
19.40 \\
15.73\end{array}$ & & $3 d^{4}\left(a^{3} \mathrm{D}\right) 4 p$ & $\left\{\begin{array}{c}w^{4} \mathbf{F}_{11 / 2}^{\circ} \\
{ }^{4} \mathbf{F}_{21 / 2}^{\circ} \\
{ }^{4} \mathbf{F}_{31 / 2}^{\circ} \\
{ }^{4} \mathbf{F}_{41 / 2}^{\circ}\end{array}\right.$ & $\begin{array}{l}74273.48 \\
74318.86 \\
74423.84 \\
74504.51\end{array}$ & $\begin{array}{r}45.38 \\
104.98 \\
80.67\end{array}$ & \\
\hline $3 d^{4}\left(a^{3} \mathrm{~F}\right) 4 p$ & $\left\{\begin{array}{r}y^{4} \mathrm{~F}_{11 / 2}^{\circ} \\
{ }^{4} \mathrm{~F}_{21 / 2}^{\circ} \\
{ }^{4} \mathbf{F}_{31 / 2}^{\circ}\end{array}\right.$ & $\begin{array}{l}67379.92 \\
67387.30 \\
67393.80\end{array}$ & $\begin{array}{r}\text { 7. } 38 \\
6.50 \\
5\end{array}$ & & $3 d^{4}\left(a^{1} \mathrm{I}\right) 4 p$ & $\left\{\begin{array}{rl}y & 2 I_{51 / 2}^{\circ} \\
2 & I_{61 / 2}^{\circ}\end{array}\right.$ & $\begin{array}{l}74421.76 \\
74424.35\end{array}$ & 2. 59 & \\
\hline & ${ }^{4} \mathrm{~F}_{41 / 2}^{3 / 2}$ & 67448. 82 & 55.02 & & $3 d^{4}\left(a^{1} \mathrm{I}\right) 4 p$ & $\left\{\begin{array}{r}x^{2} \mathrm{H}_{41 / 2}^{\circ} \\
{ }^{2} \mathrm{H}_{51 / 2}^{0}\end{array}\right.$ & $\begin{array}{l}74455.90 \\
74707.42\end{array}$ & 251.52 & \\
\hline $3 d^{4}\left(a^{3} \mathrm{H}\right) 4 p$ & $\left\{\begin{aligned} z^{2} \mathbf{I}_{51 / 2}^{\circ} \\
\\
{ }^{2} \boldsymbol{I}_{61 / 2}^{\circ}\end{aligned}\right.$ & $\begin{array}{l}\text { 67506. } 34 \\
67589.06\end{array}$ & 82.72 & & $3 d^{4}\left(a^{3} \mathrm{D}\right) 4 p$ & $\left\{\begin{array}{c}x^{4} \mathrm{P}_{21 / 2} \\
{ }^{4} \mathrm{P}_{11 / 6}^{1 / 2}\end{array}\right.$ & $\begin{array}{l}74484.25 \\
74718.05\end{array}$ & -233.80 & \\
\hline $3 d^{4}\left(a^{3} \mathrm{~F}\right) 4 p$ & $\left\{\begin{array}{r}x^{4} \mathrm{D}_{01 / 2}{ }^{1}{ }^{4} \mathrm{D}_{11 / 2}^{1} \\
{ }^{4} \mathrm{D}_{21 / 2}^{1} \\
{ }^{4} \mathrm{D}_{21 / 2}^{1}\end{array}\right.$ & $\begin{array}{l}67859.91 \\
67870.50 \\
67868.05 \\
67875.68\end{array}$ & $\begin{array}{r}10.59 \\
-2.45 \\
7.63\end{array}$ & & $3 d^{4}\left(a^{1} \mathrm{I}\right) 4 p$ & $\begin{array}{r}{ }^{4} \mathrm{P}_{01 / 2}^{11 / 2} \\
\left\{\begin{array}{c}z^{2} \mathrm{~K}_{61 / 2}^{\circ} \\
{ }^{2} \mathrm{~K}_{71 / 2}\end{array}\right.\end{array}$ & $\begin{array}{l}74920.80 \\
74743.33 \\
74958.80\end{array}$ & 215.47 & \\
\hline $3 d^{4}\left(a^{3} \mathrm{P}\right) 4 p$ & $z{ }^{4} \mathrm{~S}_{\mathrm{i} 1 / 2}$ & 68305.73 & 1. 978 & & $3 d^{4}\left(a^{3} \mathrm{D}\right) 4 p$ & $\left\{\begin{array}{c}y^{2} \mathbf{P}_{01 / 2}^{\circ} \\
{ }^{2} \mathbf{P}_{11 / 2}^{0}\end{array}\right.$ & $\begin{array}{l}\text { 74854. } 08 \\
74984.93\end{array}$ & 130.85 & \\
\hline $3 d^{4}\left(a^{3} \mathrm{H}\right) 4 p$ & $\left\{\begin{array}{c}z^{2} \mathrm{H}_{41 / 2}^{\circ} \\
{ }^{2} \mathrm{H}_{51 / 2}^{\circ}\end{array}\right.$ & $\begin{array}{l}\text { 68477. } 11 \\
68737.99\end{array}$ & 260.88 & & $3 d^{4}\left(a^{1} \mathrm{G}\right) 4 p$ & $\left\{\begin{array}{r}w^{2} \mathrm{G}_{31 / 2}^{\circ} \\
{ }^{2} \mathrm{G}_{41 / 2}^{\circ}\end{array}\right.$ & $\begin{array}{l}75716.74 \\
75810.10\end{array}$ & 93. 36 & \\
\hline $3 d^{4}\left(a^{3} \mathrm{~F}\right) 4 p$ & $\left\{\begin{array}{l}z^{2} \mathbf{F}_{21 / 2}^{\circ} \\
{ }^{2} \mathbf{F}_{31 / 2}^{o}\end{array}\right.$ & $\begin{array}{l}68583.44 \\
68760.00\end{array}$ & 176.56 & & $3 d^{4}\left(a^{1} \mathrm{G}\right) 4 p$ & $\left\{\begin{array}{r}w^{2} \mathrm{~F}_{31 / 2}^{\circ} \\
{ }^{2} \mathrm{~F}_{21 / 2}^{\circ}\end{array}\right.$ & $\begin{array}{l}76879.03 \\
76987.78\end{array}$ & -108.75 & \\
\hline $3 d^{4}\left(a^{3} \mathrm{G}\right) 4 p$ & $\left\{\begin{array}{c}y{ }^{4} \mathrm{H}_{31 / 2}^{\circ}{ }^{\circ}{ }^{4} \mathrm{H}_{41 / 2}^{\circ} \\
{ }^{4} \mathrm{H}_{51 / 2}^{\circ} \\
{ }^{4} \mathrm{H}_{611 / 2}^{\circ}\end{array}\right.$ & $\begin{array}{l}68843.51 \\
68992.55 \\
69170.60 \\
69388.40\end{array}$ & $\begin{array}{l}149.04 \\
178.05 \\
217.80\end{array}$ & & $3 d^{4}\left(\alpha^{1} \mathrm{G}\right) 4 p$ & $\left\{\begin{array}{r}w^{2} \mathrm{H}_{51 / 2}^{\circ} \\
{ }^{2} \mathrm{H}_{41 / 2}^{\circ}\end{array}\right.$ & $\begin{array}{l}77078.96 \\
77270.40\end{array}$ & -191.44 & \\
\hline & $\int x^{4} \mathrm{~F}_{11 / 2}^{\circ}$ & $\begin{array}{l}69348.36 \\
69478.06\end{array}$ & 129. 70 & & $3 d^{4}\left(a{ }^{1} \mathrm{~S}\right) 4 p$ & $\left\{\begin{array}{r}x^{2} \mathrm{P}_{11 / 2}^{\circ} \\
{ }^{2} \mathrm{P}_{01 / 2}^{\circ}\end{array}\right.$ & $\begin{array}{l}77713.66 \\
77777.58\end{array}$ & -63.92 & \\
\hline $3 d^{4}\left(a^{3} \mathrm{G}\right) 4 p$ & $\left\{\begin{array}{l}{ }^{4} \mathbf{F}_{31 / 2}^{23 / 2} \\
{ }^{4} \mathbf{F}_{41 / 2}^{\circ}\end{array}\right.$ & $\begin{array}{l}\text { 69506. } 16 \\
69498.27\end{array}$ & $\begin{array}{r}28.10 \\
-7.89\end{array}$ & & $3 d^{4}\left(a^{3} \mathrm{D}\right) 4 p$ & $\left\{\begin{array}{r}x^{2} \mathrm{D}_{21 / 2}^{\circ} \\
{ }^{2} \mathrm{D}_{11 / 2}^{\circ}\end{array}\right.$ & $\begin{array}{l}77935.24 \\
78109.64\end{array}$ & -174.40 & \\
\hline $3 d^{4}\left(a^{3} \mathrm{~F}\right) 4 p$ & $\left\{\begin{array}{r}y{ }^{2} \mathrm{D}_{11 / 2}^{\circ} \\
{ }^{2} \mathrm{D}_{21 / 2}^{\circ}\end{array}\right.$ & $\begin{array}{l}\text { 69638. } 77 \\
69954.20\end{array}$ & 315.43 & & $3 d^{4}\left(a^{1} \mathrm{D}\right) 4 p$ & $\left\{\begin{array}{r}w^{2} \mathrm{D}_{11 / 2}^{\circ} \\
{ }^{2} \mathrm{D}_{21 / 2}^{\circ}\end{array}\right.$ & $\begin{array}{l}80288.25 \\
80420.43\end{array}$ & 132. 18 & \\
\hline $3 d^{4}\left(a^{3} \mathrm{~F}\right) 4 p$ & $\left\{\begin{array}{rr}y & \mathrm{G}_{31 / 2}^{\circ} \\
& { }^{\circ} \mathrm{G}_{41 / 2}^{\circ}\end{array}\right.$ & $\begin{array}{l}\text { 69903. } 46 \\
\text { 70107. } 83\end{array}$ & 204. 37 & & $3 d^{4}\left(a^{1} \mathrm{D}\right) 4 p$ & $\left\{\begin{array}{r}v^{2} \mathrm{~F}_{21 / 2}^{\circ} \\
{ }^{2} \mathrm{~F}_{31 / 2}^{1 / 2}\end{array}\right.$ & $\begin{array}{l}81232.91 \\
81432.36\end{array}$ & 199. 45 & \\
\hline $3 d^{4}\left(a^{3} \mathrm{G}\right) 4 p$ & $\left\{\begin{array}{c}x^{4} \mathrm{G}_{21 / 2}^{\circ} \\
{ }^{4} \mathrm{G}_{31 / 2}^{\circ}\end{array}\right.$ & $\begin{array}{l}\text { 70317. } 04 \\
70427.22\end{array}$ & $\begin{array}{l}110.18 \\
252.00\end{array}$ & & $3 d^{4}\left(a^{1} \mathrm{D}\right) 4 p$ & $\left\{\begin{array}{r}w^{2} \mathrm{P}_{01 / 2}^{\circ} \\
{ }^{2} \mathrm{P}_{11 / 2}^{11 / 2}\end{array}\right.$ & $\begin{array}{l}82854.00 \\
82920.03\end{array}$ & 66. 03 & . \\
\hline & $\left(\begin{array}{l}{ }^{4} \mathrm{G}_{41 / 2}^{\circ} \\
{ }^{4} \mathrm{G}_{51 / 2}^{\circ}\end{array}\right.$ & $\begin{array}{l}70679.22 \\
70879.95\end{array}$ & 200.73 & & $3 d^{4}\left(a^{1} \mathrm{~F}\right) 4 p$ & $\left\{\begin{array}{r}u^{2} \mathbf{F}_{21 / 2}^{\circ} \\
{ }^{2} \mathbf{F}_{31 / 2}^{\circ}\end{array}\right.$ & $\begin{array}{l}84604.99 \\
84677.39\end{array}$ & 72. 40 & \\
\hline $3 d^{4}\left(a^{3} \mathrm{G}\right) 4 p$ & $\left\{\begin{aligned} y^{2} \mathrm{H}_{41 / 2}^{\circ} \\
{ }^{2} \mathrm{H}_{51 / 2}^{\circ}\end{aligned}\right.$ & $\begin{array}{l}\text { 70394. } 46 \\
70399.04\end{array}$ & 4. 58 & & $3 d^{4}\left(a^{1} \mathrm{~F}\right) 4 p$ & $\left\{\begin{array}{c}v^{2} \mathrm{G}_{31 / 2}^{0} \\
{ }^{3} \mathrm{G}_{41 / 2}^{3 / 2}\end{array}\right.$ & $\begin{array}{l}85573.43 \\
85939.50\end{array}$ & 366.08 & \\
\hline $3 d^{4}\left(a^{3} \mathrm{G}\right) 4 p$ & $\left\{\begin{aligned} & y{ }^{2} \mathbf{F}_{21 / 2}^{\circ} \\
&{ }^{2} \mathbf{F}_{31 / 2}^{\circ}\end{aligned}\right.$ & $\begin{array}{l}70584.64 \\
70852.24\end{array}$ & 267. 60 & & $3 d^{4}\left(a^{1} \mathrm{~F}\right) 4 p$ & $\left\{v^{2} \mathrm{D}_{21 / 2}^{\circ}\right.$ & 86507.38 & -3.70 & \\
\hline $3 d^{4}\left(a^{3} \mathrm{G}\right) 4 p$ & $\left\{\begin{aligned} x^{2} \mathrm{G}_{31 / 2}^{\circ} & \\
& { }^{2} \mathrm{G}_{41 / 2}^{\circ}\end{aligned}\right.$ & $\begin{array}{l}72648.79 \\
72716.91\end{array}$ & 68. 12 & & & $\left({ }^{2} \mathrm{D}_{1 \frac{1}{2}}\right.$ & 90511.08 & & \\
\hline $3 d^{4}\left(a^{3} \mathrm{D}\right) 4 p$ & $\left\{\begin{array}{c}w^{4} \mathrm{D}_{01 / 2}^{\circ} \\
{ }^{4} \mathrm{D}_{11 / 2}^{\circ} \\
{ }^{4} \mathrm{D}_{21 / 2}^{0} \\
{ }^{4} \mathrm{D}_{31 / 2}^{0}\end{array}\right.$ & $\begin{array}{l}73406.68 \\
73411.94 \\
73436.27 \\
73485.60\end{array}$ & $\begin{array}{r}5.26 \\
24.33 \\
49.33\end{array}$ & & $3 d^{4}\left(b^{3} \mathrm{~F}\right) 4 p$ & $\begin{array}{l}\left\{\begin{array}{r}u^{2} \mathrm{G}_{31 / 2}^{\circ} \\
{ }^{2} \mathrm{G}_{41 / 2}^{\circ}\end{array}\right. \\
\left\{\begin{array}{r}u^{2} \mathrm{D}_{21 / 2}^{\circ} \\
{ }^{2} \mathrm{D}_{11 / 2}^{\circ}\end{array}\right.\end{array}$ & $\begin{array}{l}90986.31 \\
91103.36 \\
91426.31 \\
91556.54\end{array}$ & -130.23 & \\
\hline
\end{tabular}


TABLE 5. Theoretical terms of $\mathrm{Cr}$ II

\begin{tabular}{|c|c|}
\hline $\begin{array}{c}\text { Electron } \\
\text { configuration } \\
1 s^{2} 2 s^{2} 2 p^{6} 3 s^{2} 3 p^{6}+\end{array}$ & Theoretical terms \\
\hline $3 d^{5}$ & 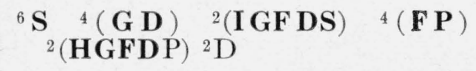 \\
\hline $3 d^{4} 4 s$ & $\begin{array}{l}{ }^{6,4} \mathbf{D}_{2(\mathrm{DGS})}^{4,2}(\mathbf{H G F D P})^{2}(\text { IGFDS })^{4,2}(\mathrm{FP}) \\
\quad\end{array}$ \\
\hline $3 d^{3} 4 s^{2}$ & ${ }^{4}(\mathbf{F P}){ }^{2}(\mathrm{HGF} \mathbf{D P}){ }^{2} \mathrm{D}$ \\
\hline $3 d^{4} 4 p$ & 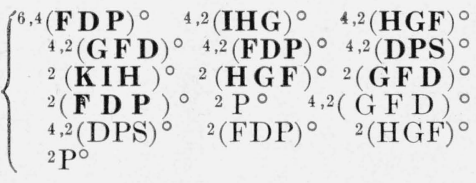 \\
\hline $3 d^{4} 4 d$ & $\begin{array}{ll}6,4 \text { (G F D P S ) } & 4,2 \text { ( K I H G F ) } \\
4,2 \text { (I H G F D ) } & 4.2 \text { ( H G F D P) } \\
4,2 \text { (GFDPS), etc. } & \end{array}$ \\
\hline $3 d^{4} 5 s$ & ${ }^{6,4} \mathbf{D}^{4,2}$ (HGFDP) etc. \\
\hline
\end{tabular}

TABle 6. Series terms of $\mathrm{Cr}$ II

\begin{tabular}{|c|c|c|c|c|c|}
\hline \multirow{2}{*}{ Terms } & \multicolumn{2}{|c|}{ Electron configuration } & \multirow{2}{*}{ Terms } & \multicolumn{2}{|c|}{ Electron configuration } \\
\hline & $3 d^{5}$ & $3 d^{4} 4 d$ & & $3 d^{4} 4 s$ & $3 d^{4} 5 s$ \\
\hline $\begin{array}{l}{ }^{6} \mathrm{~S}_{21 / 2} \\
{ }^{4} \mathrm{G}_{51 / 2} \\
{ }^{4} \mathrm{P}_{21 / 2} \\
{ }^{4} \mathrm{D}_{31 / 2} \\
{ }^{4} \mathrm{~F}_{41 / 3}\end{array}$ & $\begin{array}{r}0.00 \\
20512.75 \\
21822.86 \\
25033.95 \\
32854.46\end{array}$ & $\begin{array}{l}91954.78 \\
89508.63 \\
88923.2 \\
89621.25 \\
90851.00\end{array}$ & $\begin{array}{l}{ }^{6} \mathrm{D}_{41 / 3} \\
{ }^{4} \mathrm{D}_{31 / 2} \\
{ }^{4} \mathrm{G}_{51 / 2}\end{array}$ & $\begin{array}{l}\text { 12496. } 79 \\
\text { 20024. } 18 \\
33694.47\end{array}$ & $\begin{array}{r}83240.20 \\
84725.96 \\
105421.9\end{array}$ \\
\hline
\end{tabular}

\section{References}

[1] M. A. Catalán, Phil. Trans. Roy. Soc. London A 223, 127 (1922).

[2] H. Gieseler, Ann. Physik [IV] 69, 147 (1922).

[3] C. C. and H. K. Kiess, Science 56, 666 (1922).

[4] H. Kayser, Handbuch der Spectroscopie. S. Hirzel, Leipzig (1910) and (1934)

[5] C. C. Kiess, BS J. Research 5, 775 (1930) RP229

[6] E. Krömer, Z. Physik 52, 531 (1928).

[7] M. A. Catalán, An. Espan. Soc. Fis. Quim. 28, 611 (1930).

[8] C. C. Kiess and O. Laporte, Science 63, 234 (1926).

[9] R. W. Wood, Nature 140, 723 (1937).

[10] H. D. Curtis, J. Opt. Soc. Am. 8, 697 (1924).

[11] A. S. King, Astrophys. J. 41, 86 (1915); 60, 282 (1924).

[12] J. S. V. Allen and C. E. Hesthal, Phys. Rev. 47, 926 (1935).
[13] H. N. Russell, Proc. Nat. Acad. Sci. 11, 322 (1925).

[14] C. C. Kiess and G. Shortley, J. Research NBS 42, 183 (1949) RP1961.

[15] O. Laporte, Phys. Rev. 61, 302 (1942).

[16] O. Laporte and J. R. Platt, Phys. Rev. 61, 305 (1942).

[17] G. Racah, Phys. Rev. 63, 367 (1943).

[18] A. A. Schweizer, Phys. Rev. 80, 1080 (1950).

[19] H. N. Russell, Astrophys. J. 66, 233 (1927).

[20] A. G. Shenstone, Phil. Trans. Roy. Soc. London [A] 335, 195 (1936).

[21] H. N. Russell, J. Opt. Soc. Am. 40, 618 (1950).

Washington, February 23, 1951. 